\title{
III-V/Ge MOS Device Technologies for Low Power Integrated Systems
}

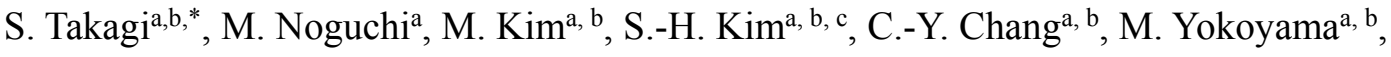 \\ K. Nishi ${ }^{a, b}$, R. Zhang ${ }^{a, d}$, M. Ke ${ }^{a, b}$ and M. Takenaka ${ }^{a, b}$ \\ E-mail: takagi@ee.t.u-tokyo.ac.jp
}

\author{
a Department of Electrical Engineering and Information Systems, The University of \\ Tokyo, 7-3-1 Hongo, Bunkyo-ku, Tokyo, 113-8656, Japan \\ b JST-CREST, 7-3-1 Hongo, Bunkyo-ku, Tokyo, 113-8656, Japan \\ ${ }^{c}$ Korea Institute of Science and Technology, Seoul 136-791, Republic of Korea \\ ${ }^{d}$ Zhejiang University, Hangzhou 310027, China
}

\section{ABSTRACT:}

CMOS utilizing high mobility III-V/Ge channels on Si substrates is expected to be one of the promising devices for high performance and low power integrated systems in the future technology nodes, because of the enhanced carrier transport properties. In addition, Tunneling-FETs (TFETs) using Ge/III-V materials are regarded as one of the most important steep slope devices for the ultra-low power applications. In this paper, we address the device and process technologies of Ge/III-V MOSFETs and TFETs on the Si CMOS platform. The channel formation, source/drain (S/D) formation and gate stack engineering are introduced for satisfying the device requirements. The plasma post oxidation to form $\mathrm{GeO}_{\mathrm{x}}$ interfacial layers is a key gate stack technology for Ge CMOS. Also, direct wafer bonding of ultrathin body quantum well III-V-OI channels, combined with Tri-gate structures, realizes high performance III-V n-MOSFETs on Si. We also 
demonstrate planar-type InGaAs and Ge/strained SOI TFETs. The defect-less $\mathrm{p}^{+}-\mathrm{n}$ source junction formation with steep impurity profiles is a key for high performance TFET operation.

Index Terms: MOSFET; Tunneling FET; Germanium; III-V semiconductors; Metal-Oxide-Semiconductor; Mobility; Interface states 


\section{INTRODUCTION}

Low power consumption is of paramount importance for advanced CMOS (Complementary Metal-Oxide-Semiconductor)-based logic LSI (Large-Scale Integrated circuits) and integrated systems mainly because of the difficulty in supply voltage $(V d d)$ reduction under realistic CMOS device design. Actually, the $V_{d d}$ lowering is the most effective way in reducing the power consumption of CMOS, $P_{\text {consum, }}$, seen in (1).

$$
P_{\text {consum }} \approx a f C_{\text {load }} V_{d d}^{2}+I_{o} \cdot 10^{-V_{\text {th }} / S} \cdot V_{d d}+I_{\text {leak }} \cdot V_{d d}
$$

where $a, f, C_{l o a d}, I 0, S$ and $I_{l e a k}$ are a constant value, the operating frequency, the load capacitance, the drain current at $V_{g}=V_{t h}$, the sub-threshold slope and the additional leakage currents including gate and junction leakages, respectively [1]. Here, the first term corresponds to the dynamic power, while the second and third terms correspond to the static power. In both components, the lowering of $V_{d d}$ can effectively reduce the power consumption.

While the steady progress in suppression of short channel effects and reduction in equivalent oxide thickness can contribute to lowering $V d d$ to some extent, the significant reduction in $V_{d d}$ is difficult for $\mathrm{Si}$ CMOS. Here, there are two possible strategies to further reduce $V_{d d}$. These strategies are schematically shown in Fig. 1. One way is to employ channel materials with higher source injection velocity such as Ge and III-V compound semiconductors. Under ballistic transport limit, on-current, Ion, can be simply represented [2-5] by

$$
I_{o n}=q N_{s} v_{i n j} \approx C_{g}\left(V_{d d}-V_{t h}\right) v_{i n j}
$$

where $q, N_{s}, v_{i n j}, C_{g}$ and $V_{t h}$ are elemental charge, surface carrier concentration at source

edge, carrier injection velocity at source edge, gate capacitance and threshold voltage, respectively. Since semiconductor channel materials with lower effective mass such as 
Ge and III-Vs are known to lead to higher injection velocity, higher $I_{o n}$ can be obtained at a given value of $V_{d d}[6,7]$. As a result, the III-V/Ge MOSFETs can reduce $N_{s}$ under a given $I_{o n}$ value, resulting in the reduction in $V_{d d}$, as shown in (2) and Fig. 1. This strategy can contribute to reduction of gate overdrive.

The typical CMOS structures composed of III-V/Ge channels are schematically shown in Fig. 2 [8-10]. Here, Ge with the significantly light hole and light electron effective mass is suitable for p-channel MOSFETs or CMOS applications. In particular, Ge CMOS is plausible in terms of the simplicity of the process/material integration, because the CMOS is composed of a single material. Also, In-based III-V semiconductors such as InGaAs, InAs and InSb with the quite light electron effective mass are suitable for n-channel MOSFET applications, while Sb-based III-V semiconductors such as GaSb, InGaSb and InSb with the light hole effective mass are suitable for p-channel MOSFETs or CMOS applications. Although III-V CMOS is another possible CMOS structure, there can still be many choices of materials for CMOS. This is because III-V materials suitable for n-MOSFETs and p-MOSFETs are different in most cases. Among them, one of the ultimate CMOS structures can be the co-integration of an In-based III-V n-MOSFET and a Ge p-MOSFET [8, 9], because of the superior physical properties including the carrier transport ones as well as the contact resistance with metals.

On the other hand, the other strategy for the reduction in $V_{d d}$ is to introduce devices with steep slope of the channel current change in sub-threshold region. This way can contribute to the reduction in $V_{d d}$ by decreasing the gate voltage swing in the sub-threshold region, as also shown in Fig. 1. Here, the inverse of the slope of the channel current change with respect to gate voltage, $V_{g}$, defined as the sub-threshold 
slope (S.S.), which is the $V_{g}$ change necessary to change channel currents by one order of the magnitude, is known to have the minimum value of $\sim 60 \mathrm{mV} / \mathrm{dec}$. at room temperature for conventional MOSFETs dominated by thermionic current in sub-threshold region. Thus, any new device operation mechanisms are needed to realize sub-threshold slope lower than this minimum value.

One of the most promising steep slope devices can be tunneling FETs (TFETs) [11-14], where tunneling probability and resulting tunneling current are modulated by $V_{g}$. Typical TFET structures are also shown in Fig. 2. This is because a variety of simulation results have reported the excellent performance of TFETs with the steep slopes $[11,13]$ and, actually, the device operations with sub-threshold slope less than 60 $\mathrm{mV} /$ dec. in a low $V_{d d}$ region typically less than $0.5 \mathrm{~V}$, have already been demonstrated without large hysteresis [15-22]. Also, fabrication of some of TFETs can be regarded as highly compatible with the Si CMOS platform. However, one of the drawbacks in TFETs is the low current drive [11-14], attributed to low tunneling probability. Particularly, Si-based TFETs are known to have the essential limitation in Ion and S.S. [11-14], because of the high bandgap energy, $E_{g}$, and the indirect bandgap. Thus, III-V/Ge is promising for the materials used in TFETs as well, because of lower $E_{g}$, direct bandgap in III-V and various possible combinations of the hetero-structures, which lead to the higher tunneling probability. Here, it is known that the source/channel junctions composed of type-II hetero-structures are effective in enhancing Ion of TFETs with maintaining low off current. It has been recognized from this viewpoint that $\mathrm{In}_{\mathrm{x}} \mathrm{Ga}_{1-\mathrm{x}} \mathrm{As} / \mathrm{GaAsy}_{\mathrm{S}} \mathrm{Sb}_{1-\mathrm{y}}$ and $\mathrm{Si} / \mathrm{Ge}$ hetero-interfaces are suitable for advanced TFETs.

As a result, the heterogeneous integration of III-V/Ge with the Si CMOS platform is a promising direction for realizing ultra-low power CMOS and TFETs along More 
Moore and Beyond CMOS approaches, respectively, in the $10 \mathrm{~nm}$ technology nodes and beyond, where ultra-low power integrated systems are indispensable. In addition, a combination of III-V/Ge-based photonic devices with advanced CMOS can also provide another possible system solution through optical interconnect for minimizing the power consumption of the interconnect under a given system performance, though this aspect is not touched on in this paper.

On the other hand, there are still many critical issues and difficult challenges for realizing III-V/Ge-based CMOS and TFETs on the Si platform [8-10, 14], which are schematically shown in Fig. 3. These include (1) formation of high-crystal-quality Ge/III-V films on Si substrates, where ultrathin body III-V-OI/GOI structures are needed with combination of any carrier transport booster technologies (2) gate stack technologies to realize superior MOS interface quality, leading to MOS gate stacks with ultrathin equivalent oxide thickness (EOT) and low densities of interface states and border traps, high channel mobility and high gate stack reliability including long term threshold voltage $\left(V_{t h}\right)$ stability (3) the formation of source/drain (S/D) with low resistivity and low leakage current for MOSFETs as well as the steep impurity profiles for TFETs (4) fabrication of ultra-short channel devices, including the optimum channel design and the formation of ultrathin body, Fin or nano-wire (NW) channels with high uniformity of the channel thickness/radius and small surface roughness and (5) total CMOS process integration including an appropriate choice of $\mathrm{n}$ - and p-channel MOSFETs and compatibility with the Si CMOS platform.

In this paper, we review the recent progress in device and process technologies of III-V/Ge MOSFETs and TFETs for solving the above critical problems on the basis of our recent research activities. We emphasize the channel formation technologies, MOS 
gate stack technologies and channel engineering technologies for III-V MOSFETs. Also, viable technologies for the superior MOS gate stack and the MOSFET performance enhancement are addressed for Ge MOSFETs. Next, we discuss key challenges for TFETs, including the appropriate material choice and the formation of the steep and high quality source junctions, which can provide both high tunneling current and low off current. We mainly present two types of planar TFETs utilizing the InGaAs channels and the Ge/strained Si hetero-structures, where Zn-diffused $\mathrm{p}^{+}$InGaAs source junctions and in-situ doping $\mathrm{p}^{+} \mathrm{Ge} / \mathrm{sSi}$, respectively, are employed for realizing steep and defect-less tunnel junction formation.

\section{LOW POWER ADVANCED CMOS TECHNOLOGIES}

\section{2-1 III-V MOSFET Technologies}

The main critical issues of the present III-V MOSFETs for the logic device applications can be high quality III-V channel formation on Si substrates, the fabrication process compatibility with the Si CMOS platform, gate stack reliability including the long term $V_{\text {th }}$ stability, realization of high performance III-V p-MOSFETs and establishment of integration scheme as CMOS. For III-V n-MOSFETs, high performance scaled MOSFETs have already been demonstrated at lower $V_{d d}$ for MOSFETs using In-based-channels such as InGaAs and InAs [23-48]. However, most of the devices have been fabricated with the recessed-channel structures on bulk III-V substrates $[23,26-29,32-37,40,42,43,46,48]$, which are not simply compatible with advanced CMOS logic technologies. While multi-gate III-V MOSFETs have also been demonstrated $[25,26,37-39,46-48]$, no clear evidence of higher performance of fully-scaled NW III-V MOSFETs, strongly expected beyond $10 \mathrm{~nm}$ technology nodes, 
has been presented yet. Also, the long term reliability of MOS gate stacks on III-V semiconductors can be regarded as a critical issue $[49,50]$, because of the existence of a large amount of slow traps.

On the other hand, the high performance of III-V p-MOSFETs with high Ion and low $I_{\text {off }}$ has not been demonstrated yet, which is mainly attributable to the poor MOS interface properties and low material qualities of Sb-based channels. As a result, viable CMOS structures using only III-V channel materials have not been fully identified yet, though several III-V CMOS devices have been reported so far [53-58].

\section{2-1-1 III-V channel formation on Si substrates}

Among a variety of difficult challenges in III-V MOSFETs listed above, one of the most difficult ones can be high quality III-V channel formation. One of the promising techniques is direct growth of III-V channels on Si substrates [59-63]. Particularly, the selective growth of limited regions of $\mathrm{Si}$ active regions [41, 46, 64-72], called an aspect ratio trapping (ART), is expected to provide a III-V channel formation process compatible with Si CMOS fabrication. Actually, the operation of InGaAs n-MOSFETs fabricated by this ART process has been demonstrated $[41,46,71]$. One of the present problems of III-V channels formed by this selective growth on Si can be inferior quality of materials including buffers, leading to high leakage current and low electron mobility [41].

Another promising approach to form III-V channels on Si is direct wafer bonding of III-V thin films on Si substrates with interfacial insulating layers working as buried oxides (BOX) [24, 30, 53, 56, 73-99]. We have proposed and demonstrated ultrathin body InGaAs-based channel formation by using direct wafer bonding [24, 30, 73-76, 
79-85, 87, 89-95, 96]. As ultrathin body $(3.2 \mathrm{~nm} \mathrm{In} 0.53 \mathrm{Ga} 0.47 \mathrm{As}) /$ ultrathin $\mathrm{BOX}\left(\mathrm{Al}_{2} \mathrm{O}_{3}\right.$ $\left.(4.4 \mathrm{~nm}) / \mathrm{SiO}_{2}(3.3 \mathrm{~nm})\right)$ channels have been realized with high material quality on $\mathrm{Si}$ $[24,76]$. Here, the lattice-matched $\operatorname{In} 0.53 \mathrm{Ga} 0.47 \mathrm{As}$ channel was epitaxially grown on a 4-inch InP substrate with interfacial $\operatorname{In} 0.53 \mathrm{Ga} 0.47 \mathrm{As} / \mathrm{InP}$ super-lattice layers as etching stop, meaning that the high material quality of the $\operatorname{In}_{0.53} \mathrm{Ga} 0.47 \mathrm{As}$ channel can be guaranteed. Thin atomic layer deposition (ALD) $\mathrm{Al}_{2} \mathrm{O}_{3}$ and $\mathrm{SiO}_{2}$ films were formed on the $\mathrm{In}_{0.53} \mathrm{Ga}_{0.47} \mathrm{As} / \mathrm{InP}$ substrate and a $\mathrm{Si}$ substrate, respectively. The $\mathrm{ALD} \mathrm{Al}_{2} \mathrm{O}_{3}$ layer is suitable for the buried oxide layer of III-V-on-insulator structures, because of the strong bonding property as well as better MOS back interface properties of III-V channel layers. After bonding of the two wafers in air, the InP substrate was selectively etched by $\mathrm{HCl}$, which can provide quite high difference in the etching rate between InP and InGaAs, allowing us to realize ultrathin InGaAs-on-insulator (-OI) structures with high thickness uniformity. We have already confirmed high electron mobility and excellent performance of MOSFETs using InGaAs-OI fabricated by the direct wafer bonding [24, $30,73-76,79-85,87,89-95,96]$.

This wafer bonding process is also applicable to other III-V channels. We have successfully fabricated GaSb-OI structures on $\mathrm{Si}$ by employing the similar wafer bonding technology $[56,98,100]$. The process flow and cross-sectional transmission electron microscope (TEM) images of the fabricated GaSb-OI on Si structure are shown in Fig. 4(a) and (b), respectively. Here, GaSb films grown on 2-inch (100) InAs wafers were used as the initial substrate. The $\mathrm{Al}_{2} \mathrm{O}_{3} \mathrm{BOX}$ layers were deposited on both $\mathrm{GaSb} / \mathrm{InAs}$ and Si wafers by ALD. After manually bonding the wafers in air, the InAs substrates were selectively etched from GaSb, resulting in formation of a 23-nm-thick $\mathrm{GaSb} / \mathrm{Al}_{2} \mathrm{O}_{3} / \mathrm{Si}$ structure with excellent $\mathrm{GaSb}$ thickness uniformity and smooth $\mathrm{GaSb}$ 
front and back interfaces. We have also confirmed the operation of p-MOSFETs using the bonded GaSb-OI structure on $\mathrm{Si}[56,98,100]$. These results suggest the versatility and the effectiveness of direct wafer bonding in terms of high quality III-V channel formation on Si substrates.

On the other hand, one essential drawback of the above process is the limited wafer size, because III-V channel layers are grown on small size III-V substrates like InP and InAs with a diameter of typically 2-4 inch. This limited wafer size makes an essential difficulty in transferring the channel layers to Si substrates with a diameter of 12 inch and beyond. In order to solve this essential problem, we have proposed and demonstrated a fabrication process of InGaAs-OI by wafer bonding of an InGaAs layer grown on a Si donor wafer instead of the one grown on an InP donor wafer $[99,101]$. By using III-V channels grown on $\mathrm{Si}$, III-V-OI structures on $\mathrm{Si}$ substrates with any available wafer size can be prepared. Recently, the similar technology has also been reported by the other group $[44,102,103]$.

Fig. 5(a) shows the process flow of InGaAs-OI wafers by the direct wafer bonding technique newly developed. Here, 4-inch Si wafers were used for the proof-of-concept demonstration of the proposed fabrication process. However, the same process is also applicable to 12-inch Si wafers. First, InGaAs channels were grown on Si wafers with InAlAs/GaAs buffer layers by Molecular Beam Epitaxy (MBE). Here, 50 -nm-thick-InGaAs films on Si show Hall electron mobility of $6550 \mathrm{~cm}^{2} / \mathrm{Vs}$ at $300 \mathrm{~K}$ with an electron concentration of $1.5 \times 10^{17} \mathrm{~cm}^{-3}$, suggesting that the good film quality is obtained even on $\mathrm{Si}$ wafers. $\mathrm{ALD} \mathrm{Al}_{2} \mathrm{O}_{3}$ was deposited on the III-V/Si wafers, followed by chemical mechanical polishing (CMP) for the surface smoothing, which is necessary for wafer bonding. After $\mathrm{HfO}_{2}$ deposition on both $\mathrm{Al}_{2} \mathrm{O}_{3} / \mathrm{III}-\mathrm{V} / \mathrm{Si}$ and $\mathrm{Al}_{2} \mathrm{O}_{3} / \mathrm{Si}$ wafers, 
the two wafers were bonded to each other. Finally, InGaAs-OI on $\mathrm{Si}$ wafers were formed by etching the top $\mathrm{Si}$, the GaAs and the InAlAs buffer layers by tetra-methyl-ammonium hydroxide (TMAH), citric acid and $\mathrm{HCl}$ based solutions, respectively. Fig. 5(b) and (c) show cross-sectional TEM images of the bonded InGaAs-OI wafer with body thickness $\left(T_{b o d y}\right)$ of $10 \mathrm{~nm}$ before and after wafer thinning, respectively. It is found that high quality and uniform 10-nm-thick InGaAs-OI, transferred from a Si donor wafer, can be formed on a Si substrate.

In order to evaluate the electrical properties of MOSFETs using the present InGaAs-OI layers on $\mathrm{Si}$, InGaAs-OI MOSFETs were fabricated with a gate stack composed of $10 \mathrm{~nm} \mathrm{Al} \mathrm{O}_{3}$ and Ta gate metal and Ni-InGaAs metal S/D. The $I_{d-} V_{g}$ characteristics of InGaAs-OI MOSFETs with $T_{b o d y}$ of $9 \mathrm{~nm}$ and $L_{G}$ of $1 \mu \mathrm{m}$ are shown in Fig. 6(a). Very good transfer curves were obtained with S.S. of $100 \mathrm{mV} / \mathrm{dec}$ and $I_{\text {on }} / I_{\text {off }}$ ratio higher than $10^{6}$. Fig. 6 (b) shows the comparison in $\mu_{\text {eff }}$ of InGaAs-OI MOSFETs at $N_{s}$ of $10^{12} \mathrm{~cm}^{-2}$ between InP and Si donor wafers as a function of $T_{b o d y}$. InGaAs-OI MOSFETs fabricated from the Si donor wafer exhibit high mobility comparable to that from the InP donor wafer, indicating that the present wafer fabrication process can realize sufficiently-high quality InGaAs-OI on Si wafers with the same level as those obtained from the InP donor wafer. Fig. 6(c) shows the cumulative distribution of leakage current $\left(I_{\text {leak }}\right)$ of the InGaAs-OI MOSFETs fabricated from both the Si and InP donor wafer at $L_{G}$ of $1 \mu \mathrm{m}$ and $V_{d}$ of $0.05 \mathrm{~V}$. Here, Ileak of MOSFETs is very sensitive to the quality of channel layers. It is found that Ileak of MOSFETs fabricated from the Si donor wafer is very uniform and even lower than that from the InP donor wafer, indicating low defect density and high material quality of fabricated InGaAs transferred from the Si substrate. These results clearly demonstrates that the proposed direct wafer 
bonding from III-V channel layers grown on $\mathrm{Si}$ can provide excellent scalability of III-V-OI wafer up to Si wafers with a diameter larger than $300 \mathrm{~mm}$ while maintaining high device quality.

\section{2-1-2 III-V MOS gate stack technology}

Formation of III-V gate stacks with thin EOT, low interface defects and realization of the sufficient long-term reliability are also quite challenging requirements. Among the long history of III-V MOS interface studies [104, 105], one of the important achievements recently obtained is the formation of better MOS interface properties of III-V channel materials such as GaAs, InGaAs and GaSb with $\mathrm{ALD} \mathrm{Al}_{2} \mathrm{O}_{3}$ and $\mathrm{HfO}_{2}$ films as gate insulators in terms of the reduction in interface state density $\left(D_{i t}\right)$ [106-114]. These improved MOS properties have been often attributed to low temperature and soft deposition by the ALD process and the self-cleaning effects of the metal-organic ALD sources on III-V native oxides or III-V surfaces [106-110], though universal understanding of the physical origin of the interface states at III-V MOS structures has not been fully obtained yet. On the other hand, the effectiveness of the ALD process on the III-V gate stack formation can contribute significantly to realizing scaled III-V MOSFETs, because of potential ultrathin EOT, applicability to multi-gate structures and mass-production friendliness.

ALD $\mathrm{Al}_{2} \mathrm{O}_{3}$ films can be regarded as quite suitable for InGaAs MOS interface stabilization. Thus, $\mathrm{HfO}_{2} / \mathrm{Al}_{2} \mathrm{O}_{3}$ stack gate insulators have been often used for InGaAs in order to simultaneously realize thin EOT and superior MOS interface properties [37, 41, 114-116]. Since thinner $\mathrm{Al}_{2} \mathrm{O}_{3}$ thickness is better in this gate stack in terms of EOT, we have studies how thin $\mathrm{Al}_{2} \mathrm{O}_{3}$ can effectively reduce $D_{i t}$ by systematically changing 
the thickness of $\mathrm{Al}_{2} \mathrm{O}_{3}$ in the $\mathrm{ALD} \mathrm{Al}_{2} \mathrm{O}_{3} / \mathrm{HfO}_{2}$ gate stacks [113]. As a result, it has been found that the insertion of only 0.2 -nm-thick ultrathin $\mathrm{Al}_{2} \mathrm{O}_{3}$ interfacial layers (ILs) for $\mathrm{HfO}_{2} / \mathrm{InGaAs}$ interfaces can effectively reduce $D_{i t}$ down to the level for sufficiently-thick $\mathrm{Al}_{2} \mathrm{O}_{3} / \mathrm{InGaAs}$ interfaces $[113,114]$. This gate stack allows us to realize $\mathrm{HfO}_{2}(2 \mathrm{~nm}) / \mathrm{Al}_{2} \mathrm{O}_{3}(0.2 \mathrm{~nm}) / \mathrm{InGaAs}$ MOS capacitors with capacitance effective thickness (CET) of $1.08 \mathrm{~nm}$ and gate leakage current of $2.4 \times 10^{-2} \mathrm{~A} / \mathrm{cm}^{2}$.

On the other hand, the remaining issues on the III-V gate stacks can be (1) the minimum $D_{i t}$ values at $\mathrm{InGaAs}$ MOS gate stacks with $\mathrm{Al}_{2} \mathrm{O}_{3}$ ILs are typically still around in lower half of $10^{12} \mathrm{~cm}^{-2} \mathrm{eV}^{-1}$ order, which are higher than in $\mathrm{Si}$ and Ge MOS gate stacks (2) MOS interface properties on Sb-based materials such as GaSb, GaAsSb and InGaSb are still poor (3) MOS gate stack reliability such as PBTI reliability seems not to be able to satisfy the realistic criterion [49-52]. While the examination of the MOS reliability and possible physical origins of reliability degradation is on-going, appropriate choices of gate stacks and treatments are still needed.

For further reduction in $D_{i t}$ at InGaAs MOS interfaces, recent studies have revealed that ALD $\mathrm{La}_{2} \mathrm{O}_{3} / \mathrm{InGaAs}_{\text {nOS }}$ interfaces can provide lower $D_{i t}$ than $\mathrm{Al}_{2} \mathrm{O}_{3} / \mathrm{InGaAs}$ ones $[117,118]$, which can provide a new MOS interface engineering scheme. Also, it has been reported that trivalent oxides such as $\mathrm{Al}_{2} \mathrm{O}_{3}, \mathrm{Y}_{2} \mathrm{O}_{3}$, and $\mathrm{La}_{2} \mathrm{O}_{3}$ can provide better interfaces with III-V semiconductors than $\mathrm{HfO}_{2}$, because of the well-arranged chemical bonding and the abrupt interfaces [119]. Thus, we have systematically examined the InGaAs MOS interface properties by using $\mathrm{ALD} \mathrm{La}_{2} \mathrm{O}_{3}$, InGaAs [118]. Fig. 7 shows the comparison in $C$ - $V$ curves of $\mathrm{Al}_{2} \mathrm{O}_{3}(3.5 \mathrm{~nm}) / \mathrm{InGaAs} \mathrm{Al}_{2} \mathrm{O}_{3}(3.5 \mathrm{~nm}) / \mathrm{La}_{2} \mathrm{O}_{3}(0.4 \mathrm{~nm}(10$ cycles) $) / \mathrm{InGaAs}$ and $\mathrm{Al}_{2} \mathrm{O}_{3}(3.5 \mathrm{~nm}) / \mathrm{La}_{2} \mathrm{O}_{3}(2.9 \mathrm{~nm}$ (40 cycles))/InGaAs MOS gate stacks with Au gate electrodes, where post metallization annealing (PMA) in $\mathrm{N}_{2}$ at 300 
${ }^{\circ} \mathrm{C}$ for 1 min was performed. Here, $\mathrm{La}(\mathrm{iPrCp})_{3}$ and $\mathrm{H}_{2} \mathrm{O}$ were used as the precursors for $\mathrm{La}_{2} \mathrm{O}_{3}$. The ALD mode deposition rate at $150{ }^{\circ} \mathrm{C}$ was $0.1 \mathrm{~nm} /$ cycle with incubation of around 7 cycles.

Fig. 8 shows $D_{\text {it }}$ of $\mathrm{Al}_{2} \mathrm{O}_{3}(3.5 \mathrm{~nm}) / \mathrm{La}_{2} \mathrm{O}_{3} / \mathrm{InGaAs}$ at the surface energy of $0.1 \mathrm{eV}$ from the midgap as a function of the $\mathrm{La}_{2} \mathrm{O}_{3}$ ALD cycle number, corresponding to the change in the $\mathrm{La}_{2} \mathrm{O}_{3}$ thickness from $0 \mathrm{~nm}$ to $2.9 \mathrm{~nm}$. Here, data of $\mathrm{La}_{2} \mathrm{O}_{3}(2.9 \mathrm{~nm}(40$ cycles))/InGaAs MOS gate stacks without $\mathrm{Al}_{2} \mathrm{O}_{3}$ has also been added in the Fig. 8. The $D_{i t}$ values were determined by the conductance method. It is found that quite low $D_{i t}$ of $\sim 3 \times 10^{11} \mathrm{~cm}^{-2} \cdot \mathrm{eV}^{-1}$, which is lower than that in the TiN/W/La2 $\mathrm{O}_{3} / \mathrm{InGaAs}$ gate stacks reported in [117], is obtained for $\mathrm{La}_{2} \mathrm{O}_{3}$ (2.9 nm (40 cycles))/ InGaAs MOS. However, the hysteresis of the $C-V$ curves becomes larger with an increase in the $\mathrm{La}_{2} \mathrm{O}_{3}$ thickness, shown in Fig. 7(b), suggesting the existence of a high density of slow traps in $\mathrm{La}_{2} \mathrm{O}_{3}$. On the other hand, reducing the $\mathrm{La}_{2} \mathrm{O}_{3}$ ILs can decrease in the hysteresis. Also, $D_{i t}$ of $\mathrm{Al}_{2} \mathrm{O}_{3}$ $(3.5 \mathrm{~nm}) / \mathrm{La}_{2} \mathrm{O}_{3}(0.4 \mathrm{~nm}) / \mathrm{InGaAs}$ is lower than that of $\mathrm{Al}_{2} \mathrm{O}_{3} / \mathrm{InGaAs}$, though $\mathrm{Al}_{2} \mathrm{O}_{3}$ ALD increases $D_{i t}$ even for 2.9-nm-thick $\mathrm{La}_{2} \mathrm{O}_{3} \mathrm{IL}$, presumably due to a reaction of Tri-methyl-aluminium (TMA) with the $\mathrm{La}_{2} \mathrm{O}_{3}$ ILs. These results indicate that ALD $\mathrm{La}_{2} \mathrm{O}_{3}$ ILs, combined with $\mathrm{Al}_{2} \mathrm{O}_{3}$, can compromise the reduction in both $D_{i t}$ and slow trap density and, thus, that $\mathrm{La}_{2} \mathrm{O}_{3}$ can work as a promising high-k IL for InGaAs gate stacks.

From the viewpoint of the realization of III-V p-MOSFETs or CMOS, on the other hand, MOS structures of Antimonide-based channels such as GaSb [30, 96, 120-127], InGaSb [53-55, 88, 100, 125, 128, 129] and InSb [130-132] have stirred a strong interest. While some of the Sb-based devices have shown the high hole mobility, the MOS interface properties are still much poorer than those of InGaAs, which must be a 
critical issue for high performance devices. In particularly, Sb-based materials with lower energy difference between the valence band edge and the vacuum level are also promising for the application of TFETs employing type-II hetero-structures, which will be described in the next section. Thus, the improvement in the MOS interfaces of the Sb-based materials is a key issue for realizing high performance of TFETs.

It is known for $\mathrm{GaSb}$ gate stacks that $\mathrm{ALD} \mathrm{Al}_{2} \mathrm{O}_{3} / \mathrm{GaSb}$ MOS interfaces have much higher $D_{\text {it }}$ than $\mathrm{Al}_{2} \mathrm{O}_{3} / \mathrm{InGaAs}$ ones, suggesting that the effect of the TMA cleaning effect might not be effective for GaSb surfaces. It has been reported [133] that the GaSb MOS Interface properties are sensitive to the ALD temperature, which must be lower than $200{ }^{\circ} \mathrm{C}$ for obtaining better MOS interfaces. In addition, one of the promising ILs for passivating GaSb surfaces is ultrathin InAs layers [134-136]. Here, the choice of the InAs thickness is important, because too thick InAs can simply work as an InAs channel. In addition, the ultrathin InAs can change from the broken band lineup of InAs/GaSb to the type-II staggered one because of the quantum confinement effect of electrons in the conduction band of InAs, leading to suppression of a leakage path through the broken-gap InAs/GaSb interface [56]. Fig. 9 shows the $C-V$ curves of the $\mathrm{Al}_{2} \mathrm{O}_{3} / \mathrm{InAs} / \mathrm{p}-\mathrm{GaSb} \mathrm{MOS}$ capacitors with the InAs thickness of $0,0.5,1.5$ and $3 \mathrm{~nm}$, measured at room temperature. Here, the unintentionally-doped InAs layers were grown on p-type (100) GaSb wafers with carrier concentration $\left(\mathrm{N}_{\mathrm{A}}\right)$ of $\sim 1 \times 10^{17} \mathrm{~cm}^{-3}$ by metal organic chemical vapor deposition (MOCVD). After that, 5-nm-thick $\mathrm{ALD} \mathrm{Al}_{2} \mathrm{O}_{3}$ layers were deposited on the InAs/GaSb wafers at $150{ }^{\circ} \mathrm{C}$, after the pre-cleaning using an $\left(\mathrm{NH}_{4}\right)_{2} \mathrm{~S}_{\mathrm{x}}$ solution. The capacitance modulation with changing gate voltage is limited for the MOS capacitors with 0 - and 0.5-nm InAs passivation, suggesting large $D_{i t}$. On the other hand, the $\mathrm{C}-\mathrm{V}$ curves are found to improve with an increase in the InAs thickness 
up to $1.5 \mathrm{~nm}$, demonstrating the effectiveness of the InAs passivation on the GaSb MOS interface properties.

Fig. 10 shows the relationship between $D_{i t}$ and the surface energy from the midgap energy as a parameter of the InAs thickness of $0,0.5,1.0,1.5$, and $2.0 \mathrm{~nm}$. Here, $\mathrm{D}_{\text {it }}$ was evaluated from the $1 \mathrm{MHz} C-V$ curves at $100 \mathrm{~K}$ by using the Terman method. The $\mathrm{Al}_{2} \mathrm{O}_{3} / \mathrm{InAs}(1.5 \mathrm{~nm}) / \mathrm{p}-\mathrm{GaSb}$ MOS capacitors exhibits the lowest minimum $D_{i t}$ of $\sim 6.6 \times 10^{12} \mathrm{~cm}^{-2} \mathrm{eV}^{-1}$ at $-0.24 \mathrm{eV}$, which is reduced by $\sim 50 \%$ from that of $\sim 1.4 \times 10^{13}$ $\mathrm{cm}^{-2} \mathrm{eV}^{-1}$ in the $\mathrm{Al}_{2} \mathrm{O}_{3} / \mathrm{p}-\mathrm{GaSb}$ MOSCAPs. These results indicate that InAs ILs with an appropriate thickness can significantly improve the GaSb MOS interface properties, probably due to suppression of $\mathrm{GaSb}$ surface oxidation and/or $\mathrm{Sb}$ sublimation from the surfaces by the InAs ILs. However, further careful optimization of the gate stack formation process and the demonstration of much lower $D_{i t}$ and higher effective mobility are still strongly needed for convincingly proving that GaSb-based MOS devices are really promising for future logic applications.

\section{2-1-3 III-V channel engineering technology}

In order to apply III-V channels to the scaled CMOS, suppression of short channel effects (SCE) is mandatory, in addition to high carrier injection velocity. Form this viewpoint, ultrathin body structures as well as multi-gate structures like FinFETs and NW FETs must also be embodied by III-V channels on Si without any degradation of the material and interface qualities. Actually, there have recently been many reports on III-V-channel FinFETs [37, 44], Tri-gate MOSFETs [25, 37-39, 41] and NW MOSFETs $[26,46-48,71]$. We have focused on ultrathin-body III-V-OI structures on Si wafers [8, $24,30,31,38,39]$, fabricated by wafer bonding, with thickness below $10 \mathrm{~nm}$. One of 
the strong concerns in such thin channels is the mobility reduction with decreasing $T_{b o d y}$, attributable to the body thickness fluctuation scattering $[137,138]$. In order to mitigate this mobility degradation due to thickness fluctuation of a single InGaAs-OI layer, two types of channel structure engineering have been employed [30, 38, 87, 94, 139]. One consists in increasing the In content of main InGaAs channels up to pure InAs and the other is the introduction of MOS interface buffers, where the higher-In-content main channels are sandwiched by lower-In-content InGaAs buffers having front and back MOS direct interfaces. By employing this quantum well (QW) channel structure, the wave function of electrons can be confined more into the center of the channels with higher In content, resulting in higher mobility due to lower effective mass in the InGaAs channel with higher In content, reduction in Coulomb scattering by MOS interface charges and mitigation of the influence of the QW total thickness fluctuation $[94,139]$. It has been found that n-MOSFETs with an $\operatorname{In}_{0.3} \mathrm{Ga}_{0.7} \mathrm{As}(3 \mathrm{~nm}) / \mathrm{InAs}(3 \mathrm{~nm}) / \mathrm{In}_{0.3} \mathrm{Ga}_{0.7} \mathrm{As}$ (3nm)-OI channel, fabricated on Si substrates, provides high electron mobility of 3180 $\mathrm{cm}^{2} / \mathrm{Vs}$ at $300 \mathrm{~K}$. We have also demonstrated operation of MOSFETs with the channel length $\left(L_{c h}\right)$ of $20 \mathrm{~nm}$ using this channel structure on $\mathrm{Si}$, exhibiting high $I_{o n}$ of 2.4 $\mathrm{mA} / \mu \mathrm{m}$ at $V d d$ of $0.5 \mathrm{~V}[31,140]$.

In order to realize high performance InAs MOSFETs with further improved SCE immunity, Tri-gate $\operatorname{In}_{0.3} \mathrm{Ga} 0.7 \mathrm{As} \quad(3 \mathrm{~nm}) / \mathrm{InAs} \quad(3 \mathrm{~nm}) / \quad \mathrm{In}_{0.3} \mathrm{Ga} 0.7 \mathrm{As} \quad(3 \mathrm{~nm})-\mathrm{OI} \quad \mathrm{QW}$ MOSFETs have been fabricated by narrowing the gate width of the InGaAs-OI channels $[38,39]$. Fig. 11 shows the device structure and the schematic process flow. A Fin-like tri-gate channel structure with top gate width $\left(W_{G}\right)$ of down to $36 \mathrm{~nm}$ and (111) facet side channel surfaces were formed by chemical wet etching of InGaAs layer. A top view Scanning Electron Microscope (SEM) photograph of a tri-gate MOSFET with a narrow 
channel width is also shown in Fig. 11. Here, $\mathrm{Ta} / \mathrm{Al}_{2} \mathrm{O}_{3}$ gate stack and Ni-InGaAs metal S/D were employed.

Fig. 12(a) shows the $I_{d}-V_{g}$ characteristics of the In0.3 Ga0.7As/InAs/In0.3Ga0.7As-OI tri-gate MOSFETs with $L_{c h}$ of $20 \mathrm{~nm}$ as a parameter of $W_{G}$ of 40, 50, 140, and $360 \mathrm{~nm}$. Severe SCEs were observed in the MOSFETs with $W_{G}$ wider than $100 \mathrm{~nm}$, whereas SCEs were effectively suppressed in MOSFETs with narrower $W_{G}$. The effect of the tri-gate structure on SCE is more clearly seen in drain-induced barrier lowering (DIBL) of MOSFETs. Fig. 12(b) shows the characteristics of DIBL with different $W_{G}$ as a function of $L_{c h}$. While the severe DIBL is observed for the InGaAs-OI tri-gate MOSFETs with wide $W_{G}$ in $L_{c h}$ less than $100 \mathrm{~nm}$, DIBL becomes much reduced with $W_{G}$ narrower than $50 \mathrm{~nm}$. These results strongly indicate that the side gate effect with narrow $W_{G}$ in tri-gate MOSFETs can significantly suppress SCEs in $L_{c h}$ less than $50 \mathrm{~nm}$.

Fig. 12(c) shows $I_{o n}$ at $V_{d d}$ of $0.5 \mathrm{~V}$ and $I_{\text {off }}$ of $100 \mathrm{nA} / \mu \mathrm{m}$ characteristics with different $W_{G}$ as a parameter of $L_{c h}$. Here, the gate voltage was shifted from the measured $I_{D}-V_{G}$ characteristics so as to provide $I_{\text {off }}$ of $100 \mathrm{nA} / \mu \mathrm{m}$. $I_{\text {on }}$ of $\sim 380 \mu \mathrm{A} / \mu \mathrm{m}$ has been obtained under $V_{d}$ and $V_{g}$ of $0.5 \mathrm{~V}$. On the other hand, the saturation behavior of $I_{o n}$ in short $L_{c h}$ is attributed to relatively large $\mathrm{S} / \mathrm{D}$ resistance of $410 \Omega \mu \mathrm{m}$ in the present devices, caused by the long distance between the gate and the pad electrodes, and unintentional thinning of the $\mathrm{Ni}-\mathrm{InGaAs} \mathrm{S} / \mathrm{D}$ layers due to over etching of unreacted $\mathrm{Ni}$ during the device fabrication. Thus, the optimizations of the device geometry and the process conditions are expected to provide further enhancement of device performance.

Fig. 13 shows a benchmark of $I_{o n}$ at $V_{d}$ of $0.5 \mathrm{~V}$ with gate overdrive of $0.5 \mathrm{~V}$, taken from the present In ${ }_{0.3} \mathrm{Ga} 0.7 \mathrm{As} / \mathrm{InAs} / \mathrm{In}_{0.3 \mathrm{Ga}} \mathrm{GAs}$ As-OI tri-gate MOSFETs $[38,39]$ and reported advanced InGaAs MOSFETs [25-29, 32, 35-37, 40-48]. Here, $\mathrm{V}_{\mathrm{g}}$ has been 
shifted so that $I_{\text {off }}$ at gate overdrive of $0 \mathrm{~V}$ amounts to $100 \mathrm{nA} / \mu \mathrm{m}$ in order to guarantee the suppression of SCEs. The present devices are found to exhibit high level $I_{o n}$, obtained among the advanced InGaAs MOSFETs with shorter $L_{c h}$.

\section{2-2 Ge MOS Technologies}

\section{2-2-1 Ge MOSFET technologies}

Ge MOSFETs have been intensively studied in recent years and more mature device technologies have been demonstrated, compared to the III-V ones. In particular, Ge p-MOSFETs seem close to real applications, because good performance of short-channel Ge and high-Ge content SiGe FinFETs have already been demonstrated [141-149]. The relatively high $I_{\text {off }}$ can be one of remaining problems as low power applications. Also, higher performance of NW MOSFETs [149-152] would need to be demonstrated for future scaled technology nodes.

In contrast, Ge n-MOSFETs and resulting CMOS are still suffering from lower Ion and poorer MOS gate stack reliability, though intensive developments on Ge n-MOSFETs and CMOS have been conducted [153-185]. Here, a key technology to realize high performance Ge n-MOSFETs with high $I_{o n}$ is superior MOS interface control and gate stack technologies satisfying the requirements of thin EOT, high channel mobility, low S.S. and high gate stack reliability. Among them, MOS gate stack reliability can also be regarded as a critical problem for Ge p-MOSFETs without any Si passivation. As a result, MOS interface control engineering is still of paramount importance for Ge MOSFETs.

\section{2-2-2 Ge MOS gate stack technology}


Among a variety of gate insulators and/or interface control layers on Ge, attention has recently been paid to $\mathrm{GeO}_{2} / \mathrm{Ge}$-based interfaces $[150-156,158-176,179-183,185-213]$, because of the superior interface properties and resulting high carrier mobility in Ge MOSFETs. Particularly, high electron mobility can be obtained only for Ge n-MOSFETs with $\mathrm{GeO}_{2} / \mathrm{Ge}$ interfaces $[153-156,158-176,179-183]$. In order to realize low $D_{i t}$ and thin EOT at the same time for Ge-oxide-based ILs, we have proposed an IL formation process employing Electron cyclotron resonance (ECR) oxygen plasma to form $\mathrm{GeO}_{x}$ ILs after thin $\mathrm{ALD} \mathrm{HfO}_{2} / \mathrm{Al}_{2} \mathrm{O}_{3}$ stacks [162-164, 166, 204-207]. The basic process flow is shown in Fig. 14. Here, $\mathrm{Al}_{2} \mathrm{O}_{3}$ serves as a sufficient oxygen barrier that suppresses the growth of unnecessarily-thick $\mathrm{GeO}_{\mathrm{x}} \mathrm{IL}$, thanks to its intrinsic oxygen permeability, as well as a passivation layer that prevents Ge MOS interfaces from ECR plasma damages and process damages during the successive fabrication processes.

Figure 15 shows EOT and $D_{i t}$ of the $\mathrm{Au} / \mathrm{HfO}_{2}(2.2 \mathrm{~nm}) / \mathrm{Al}_{2} \mathrm{O}_{3}(0.2 \mathrm{~nm}) / \mathrm{GeO}_{\mathrm{x}} / \mathrm{Ge}$ MOS capacitors at $-0.2 \mathrm{eV}$ from the midgap as a function of the plasma post oxidation time. It is found that high $D_{i t}$ of $\sim 10^{12} \mathrm{~cm}^{-2} \mathrm{eV}^{-1}$ in the $\mathrm{HfO}_{2} / \mathrm{Al}_{2} \mathrm{O}_{3} / \mathrm{Ge}$ gate stack without plasma post oxidation rapidly decreases even with a very short term plasma exposure of $5 \mathrm{~s}$ and continuously decreases with an increase in the plasma oxidation time. In contrast, EOT increases only from 0.72 to $0.82 \mathrm{~nm}$ with the plasma post oxidation of 25 s. As a result, ECR plasma oxidation can sufficiently reduce $D_{i t}$ at small expense of the increase of EOT less than $0.1 \mathrm{~nm}$.

It has been observed, on the other hand, that ECR plasma oxidation of $\mathrm{HfO}_{2} / \mathrm{Ge}$ direct stacks provides inferior MOS interface properties with high $\mathrm{D}_{\mathrm{it}}$, attributed to the inter-mixing of $\mathrm{HfO}_{2}$ and $\mathrm{GeO}_{x}$, and the higher $\mathrm{Hf}$ content at MOS interfaces. Thus, the insertion of ultrathin $\mathrm{Al}_{2} \mathrm{O}_{3}$ films is needed as an inter-diffusion control layer against 
$\mathrm{HfO}_{2}$. The results of Fig. 15 indicate that 0.2-nm-ultrathin $\mathrm{Al}_{2} \mathrm{O}_{3}$ can suppress the inter-diffusion and form $\mathrm{GeO}_{x}$ ILs between $\mathrm{Al}_{2} \mathrm{O}_{3}$ and $\mathrm{Ge}$, maintaining the effectiveness of ECR post plasma oxidation even for $\mathrm{HfO}_{2}$-based gate stacks.

One of the remaining critical issues for the Ge MOS gate stacks is the existence of a large amount of slow traps and the resulting poor BTI (bias-temperature instability) characteristics $[49,50,162,214]$, as described above. Fig. 16 shows examples of the influence of slow traps on MOSFET characteristics. The amount of hysteresis in forward and backward scan sweep of $I_{d}-V_{g}$ characteristics of Ge n- and p-MOSFETs with $\mathrm{Al}_{2} \mathrm{O}_{3} / \mathrm{GeO}_{\mathrm{x}} / \mathrm{Ge}$ gate stacks and the time dependence of $I_{d}$ are shown in Fig. 16(a) and (b), respectively. The larger hysteresis and larger current drift are observed for n-MOSFETs, meaning that a larger amount of slow traps exist near the conduction band edge of Ge than the valence band edge.

While the physical origin of these slow traps is attributable to any structural defects in gate insulators including Ge oxides, $\mathrm{Al}_{2} \mathrm{O}_{3}[49,50]$ and/or $\mathrm{HfO}_{2}$, it has not been identified yet. However, the reduction in the amount of slow traps responsible for BTI and the significant improvement in the reliability lifetime are mandatory for applying Ge MOSFETs to real applications. It has been recently reported that Y-doped $\mathrm{GeO}_{\mathrm{x}}$ interface layers fabricated by sputtering can provide the MOS interface properties with small hysteresis and low $D_{i t}[168,169,183,215]$. These results suggest that appropriate doping of $\mathrm{Y}$ atoms into $\mathrm{Ge}$ oxides and $\mathrm{Al}_{2} \mathrm{O}_{3}$ near Ge interfaces can reduce the slow trap density and improve the BTI reliability. Thus, we have examined the impact of ALD $\mathrm{Y}_{2} \mathrm{O}_{3}$ and $\mathrm{AlYO}_{3}$ gate stacks on the slow trap density [216].

Fig. 17 shows $\mathrm{C}-\mathrm{V}$ curves of $1.5-\mathrm{nm}$-thick $\mathrm{Al}_{2} \mathrm{O}_{3} / \mathrm{GeO}_{\mathrm{x}} / \mathrm{p}-\mathrm{Ge}$ with plasma post oxidation, 1.5-nm-thick $\mathrm{AlYO}_{3} / \mathrm{p}$-Ge $\mathrm{MOS}$ capacitors without plasma post oxidation 
and 1.5-nm-thick $\mathrm{AlYO}_{3} / \mathrm{GeO}_{x} / \mathrm{p}$-Ge MOS capacitors with plasma post oxidation. Here, $\mathrm{AlYO}_{3}$ was deposited as an ALD mode by alternatively supplying $\mathrm{Al}\left(\mathrm{CH}_{2}\right)_{3}$ and $(\mathrm{CpMe})_{3} \mathrm{Y}$ cycle by cycle. Since pure $\mathrm{Y}_{2} \mathrm{O}_{3} / \mathrm{Ge}$ gate stacks with and without plasma post oxidation were observed in this study to exhibit large hysteresis and thus have no improvement in the slow trap density, the properties of $\mathrm{AlYO}_{3} / \mathrm{Ge}$ gate stacks were mainly studied. It is observed in Fig. 17 that $\mathrm{AlYO}_{3} / \mathrm{GeO}_{\mathrm{x}} / \mathrm{Ge}$ has smaller hysteresis than $\mathrm{Al}_{2} \mathrm{O}_{3} / \mathrm{GeO}_{\mathrm{x}} / \mathrm{Ge}$ with plasma post oxidation and 1.5 -nm-thick $\mathrm{AlYO}_{3} / \mathrm{Ge} \mathrm{MOS}$ capacitors without plasma post oxidation.

$D_{i t}$ and slow trap areal density, $\Delta N_{f i x}$, were quantitatively compared among these three MOS interfaces by the conductance method and the hysteresis measurement $[49,50]$, respectively. Fig. 18(a) shows the energy distribution of $D_{i t}$ at the $\mathrm{AlYO}_{3} / \mathrm{Ge} \mathrm{MOS}$ interface before and after plasma post oxidation. It is confirmed that plasma posit oxidation is also effective in reducing $D_{i t}$ at the $\mathrm{AlYO}_{3} / \mathrm{Ge}$ MOS interface down to almost the same level of as that in the conventional $\mathrm{Al}_{2} \mathrm{O}_{3} / \mathrm{GeO}_{\times} / \mathrm{Ge}$ interfaces with plasma post oxidation. Fig. 18 (b) also shows $\Delta N_{f i x}$ of $1.5-\mathrm{nm}$-thick $\mathrm{AlYO} 3 / \mathrm{GeO}_{\mathrm{x}} / \mathrm{p}-\mathrm{Ge}$ and n-Ge MOS capacitors and 1.5-nm-thick $\mathrm{Al}_{2} \mathrm{O}_{3} / \mathrm{GeOx} / \mathrm{n}-\mathrm{Ge}$ and $\mathrm{p}-\mathrm{Ge} \mathrm{MOS}$ capacitors as a function of the effective electric field across gate insulators, $E_{o x}$ given by $\left(\left|V_{\text {gmin }}-V_{F B}\right|\right) /$ CET). Here, $\Delta N_{f i x}$ was evaluated from the amount of hysteresis in $C-V$ curves with changing the minimum gate voltage, $V_{g m i n}$, with keeping the maximum voltage constant, according to $[49,50]$. It is found that $\Delta N_{f i x}$ of the $\mathrm{AlYO}_{3} / \mathrm{GeO}_{\mathrm{x}} / \mathrm{p}-\mathrm{Ge}$ interface becomes lower than that of $\mathrm{Al}_{2} \mathrm{O}_{3} / \mathrm{GeO}_{\times} / \mathrm{p}-\mathrm{Ge}$ interface, while the reduction in $\Delta N_{\text {fix }}$ for $\mathrm{n}-\mathrm{Ge}$ is small for the $\mathrm{AlYO}_{3} / \mathrm{GeO}_{\mathrm{x}} / \mathrm{Ge}$ interface. These results mean that $\mathrm{AlYO}_{3}$-based gate stack can reduce the amount of slow traps near the valence band of 
Ge against the conventional $\mathrm{Al}_{2} \mathrm{O}_{3} / \mathrm{GeO}_{\mathbf{x}} / \mathrm{Ge}$ interfaces. It is also confirmed that the slow trap density near the conduction band edge is much higher than that that near the valence band edge. Thus, further improvements on the Ge gate stacks are still strongly needed to reduce the slow trap density, particularly, near the conduction band edge.

\section{2-2-3 Ge MOSFET performance improvement}

Thanks to the recent progress in Ge gate stack technologies, high mobility and high on-current of Ge n- and p-MOSFETs have been demonstrated by many groups. Among them, the gate stacks with Ge-oxide-based ILs can be regarded as the unique MOS interfaces leading to high electron mobility in Ge n-MOSFETs, attributed to lower $D_{i t}$ and interface defects near the conduction band edge. Note that Si-passivation ILs [217-222], well known as effective in high hole mobility in p-MOSFETs, would not seem to work well for n-MOSFETs, because of quite small conduction band discontinuity and no electron confinement at $\mathrm{Si} / \mathrm{Ge}$ interfaces, though the improvement of Ge n-MOSFETs with Si-passivated MOS interfaces has been still pursued [184]. We have also demonstrated the operation of $n-$ and p-MOSFETs with the $\mathrm{HfO}_{2} / \mathrm{Al}_{2} \mathrm{O}_{3} / \mathrm{GeO}_{\mathrm{x}} / \mathrm{Ge}$ gate stacks, shown in Fig. 16, under EOT of $0.76 \mathrm{~nm}[164,166]$. Fig. 19 shows $I_{d}-V_{g}$ characteristics of (100) Ge p- and n-MOSFET with the $\mathrm{HfO}_{2}(2.2$ $\mathrm{nm}) / \mathrm{Al}_{2} \mathrm{O}_{3}(0.2 \mathrm{~nm}) / \mathrm{GeO}_{\mathrm{x}}(0.35 \mathrm{~nm}) / \mathrm{Ge}$ gate stack having EOT of $0.78 \mathrm{~nm}$ at $V_{d}$ of 50 and $200 \mathrm{mV}$. Well-behaving $I_{d-} V_{g}$ curves are demonstrated for these MOSFETs, with $I_{\text {on }} / I_{\text {off }}$ ratio of $\sim 10^{4}$ for both $\mathrm{p}$ - and n-MOSFETs. The values of S.S. of the $\mathrm{p}$ - and n-MOSFETs are 90 and $85 \mathrm{mV} / \mathrm{dec}$, respectively, indicating the sufficient passivation of interface traps on the $\mathrm{GeO}_{x} / \mathrm{Ge}$ MOS interfaces

We have confirmed that high electron and hole mobility are obtained in comparison 
with the thick $\mathrm{GeO}_{2} / \mathrm{Ge}$ mobility and the $\mathrm{Si}$ mobility, particularly in high $N_{s}$ region, in spite of this ultrathin EOT. The peak electron and hole mobility of 689 and $546 \mathrm{~cm}^{2} / \mathrm{Vs}$ have been obtained. These results strongly demonstrate that $\mathrm{HfO}_{2} / \mathrm{Al}_{2} \mathrm{O}_{3} / \mathrm{GeO}_{\mathrm{x}} / \mathrm{Ge}$ gate stacks can realize high performance Ge $n$ - and p-MOSFETs with minimal mobility degradation under aggressive EOT scaling.

Recently, we have found two important factors for further improving the effective mobility of Ge MOSFETs with $\mathrm{GeO}_{\mathrm{x}}$ ILs. One is the relationship between the plasma oxidation temperature and MOS interface roughness. TEM analyses and the comparison with the theoretical surface roughness mobility through the experimental MOS interface geometry taken from the real TEM data $[164,170,173,223]$ have revealed that plasma oxidation at room temperature reduces $\mathrm{GeO}_{\mathrm{x}} / \mathrm{Ge}$ MOS interface roughness, leading to the increase in the effective mobility in high $N_{s}$ region, where surface roughness scattering is dominant. By lowering plasma post oxidation temperature from $300{ }^{\circ} \mathrm{C}$ to room temperature, the electron and hole effective mobility at $N_{s}$ of $10^{13} \mathrm{~cm}^{-2}$ have been improved by $20 \%$ and $25 \%$, respectively, as a consequence of the reduction in the MOS interface roughness.

The other finding is the effectiveness of atomic deuterium (D) annealing on the reduction in $D_{i t}$ inside the conduction and valence bands $[164,173]$ and the resulting increase in the effective mobility $[167,185]$. Fig. 20(a) shows the effective and Hall electron mobility in $\mathrm{Al}_{2} \mathrm{O}_{3} / \mathrm{GeO}_{\mathrm{x}} / \mathrm{Ge}$-MOSFETs after post deposition annealing (PDA) at $400{ }^{\circ} \mathrm{C}$, in $\mathrm{N}_{2}$ ambient or with atomic $\mathrm{D}$, in comparison with the effective mobility reported previously $[159,160,168]$. It is found that the electron effective mobility in the high $N_{s}$ region is enhanced after the atomic D PDA. On the other hand, the Hall mobility, which is much higher than the effective mobility, does not change with and without 
atomic D PDA, indicating that the carrier transport properties and scattering probability do not change with atomic D PDA. Note that the effective mobility determined by the split $C-V$ method is affected by trapping of carriers into traps under the inversion condition, while Hall measurements allow us to directly evaluate free electron concentration.

Actually, we can quantitatively evaluate the trapped electron concentration as a function of surface potential by comparing $N_{s}$ evaluated by the split $C-V$ and Hall measurements under an assumption of the Hall factor of unity. Fig. 20(b) shows the energy distributions of $D_{i t}$ inside the conduction band, evaluated by Hall measurements for $\mathrm{Al}_{2} \mathrm{O}_{3} / \mathrm{GeO}_{\mathrm{x}} / \mathrm{Ge}$ n-MOSFETs with PDA in $\mathrm{N}_{2}$ and atomic $\mathrm{D}$ ambient. It is found that a large amount $D_{i t}$ exist inside the conduction band, which leads to the significant reduction in the effective mobility in high $N_{s}$ region. The existence of similar amounts of $D_{i t}$ inside the band has been confirmed in the Ge valence band $[164,173]$ and InGaAs conduction band [224-226], which has been regarded as one of the important mobility degradation factors in high $N_{s}$ region. It is found that atomic D PDA can reduce the amount of $D_{i t}$, which is the origin of the increase in the effective mobility with atomic D PDA. These results strongly suggest that further deeper understanding of the mechanisms limiting the inversion-layer mobility in Ge MOSFETs or any MOS interface is still necessary, and possible mitigation of these limiting factors is expected to further enhance the performance of Ge MOSFETs.

\section{Tunneling FET TECHNOLOGIES}

3-1 Importance and critical issues of Tunneling FETs (TFETs)

As described in the introduction, devices enabling steeper slopes than conventional 
MOSFETs are also strongly required to realize ultralow power circuit operation with much reduced $V d d$. Among a variety of the proposals on such steep slope devices, TFETs based on the band-to-band tunneling mechanism can be regarded as one of the most promising ones. This is because the principle of the device operation can promise TFETs to reduce S.S. down to less than $\sim 60 \mathrm{mV} / \mathrm{dec}$. at room temperature, the minimum value of MOSFETs, through the combination of the tunneling distance modulation by gate voltage with the filtering effect of the tunneling electron energy due to the necessity of overlapping the energy distributions of density-of-states (DOS) between the conduction and valence bands of semiconductors [11-13]. In addition to the many simulation results verifying this concept, $[11-13,227]$ the operations with S.S. less than $60 \mathrm{mV} / \mathrm{dec}$. have been experimentally demonstrated at low $\mathrm{V}_{\mathrm{dd}}(<$ typically $0.5 \mathrm{~V})$ without hysteresis. Also, while many TFET structures have been proposed, the typical ones are composed simply of reversed p-n junctions of homo- or hetero-interface semiconductors with MOS gate stacks, which can be compatible with the present CMOS platform.

However, the present TFETs have still hold many problems and challenges listed as follows; (1) Si TFETs have lower $I_{o n}$ because of the comparatively high bandgap and reduced tunneling probability due to indirect tunneling (2) In p-n junction type TFETs, the tunneling distance is not sufficiently small in some cases because of the graded source impurity profiles, leading to lower $I_{o n}(3)$ Generation-recombination centers in semiconductors, interface defects at MOS interfaces and tail states within the bandgap of semiconductors, existing in real TFETs, significantly degrade S.S and Ioff through Shockley-Read-Hall (SRH) carrier generation mechanism and trap-assisted tunneling (TAT). In particular, this issue is serious for any other new materials including III-V/Ge 
and 2-dimensional materials than Si. (4) Insensitive and ineffective control of channel potentials at tunneling points by gate voltage due to un-optimized device structures and/or higher MOS interface defects also significantly degrades S.S. The increase in controllability of channel potential by gate potential can be basically realized by similar device engineering as used to suppress SCE in advanced CMOS devices, such as such ultrathin body structures, multi-gate structures and thin EOT of gate stacks.

As a consequence, the drive current level of reported TFETs exhibiting S.S. less than $60 \mathrm{mV} / \mathrm{dec}$ [15-22] is still quite low and resulting averaged S.S. over drive current range needed for realistic applications is still too high. Also, TFETs with comparatively high drive current have a tendency to yield higher $I_{\text {off }}$ and S.S. values. Note that low $I_{\text {off }}$ and high $I_{o n} / I_{\text {off }}$ ratio are quite critical to ultra-low power applications at which TFETs are aiming. Thus, any-TFET-specific engineering for achieving the optimum materials, device structures and fabrication process must be strongly pursued for simultaneously realizing both low S.S. of sub-60 $\mathrm{mV} / \mathrm{dec}$. over a wide range of the current level, low $I_{\text {off }}$ and high $I_{o n} / I_{\text {off }}$ ratio.

Fig. 21 schematically summarized our device/material engineering for solving these critical issues and challenges of TFETs [14]. One of the key issues is the channel material engineering for enhancing tunneling current with maintaining low $I_{\text {off. }}$ Since the sufficient tunneling current would not be able to be obtained for Si with the large $E_{g}$ and the indirect bandgap allowing phonon-assisted tunneling, other semiconductors with narrower bandgaps like Ge, InGaAs and InAs are promising for increasing Ion, because of the higher tunneling probability due to narrower $E_{g}$ and the direct bandgap for III-V semiconductors. On the other hand, one of possible drawbacks with source homo-junction TFETs with narrower $E_{g}$ is the increase in $I_{o f f}$ and the degradation in 
$I_{o n} / I_{\text {off }}$ due to the higher generation-recombination rate.

In order to solve this trade-off problem in the bandgap, a type-II hetero-structure source junction is effective in reducing tunneling distance and increasing $I_{o n}$ without reduction of the bandgap in the drain junction, which would lead to the increase in Ioff. Fig. 22(a) and (b) schematically show the band lineup of the type-II hetero-structure and a band diagram of the source $\mathrm{p}-\mathrm{n}$ junction region composed of the type-II hetero-structure. It is confirmed here that the potential barrier against electrons tunneling from the source semiconductor to the channel semiconductor (effective bandgap), Egeff, can be reduced from both bandgaps of the source and channel semiconductors, $E_{g 1}$ and $E_{g 2}$, thanks to the valence and conduction band discontinuity in the type-II hetero-structure, $\Delta \mathrm{E}_{\mathrm{c}}$ and $\Delta \mathrm{E}_{\mathrm{v}}$, indicating that higher tunneling probability is expected with maintain low source and drain junction leakage currents, directly associated with $E_{g 1}$ and $E_{g 2}$.

The second important engineering issue is to demonstrate a superior MOS gate stack formation yielding thin EOT and low MOS interface defect density at the same time. While this engineering is basically similar to that in advanced CMOS technologies, the realization of such gate stacks is still challenging for semiconductors other than Si. In addition, a novel requirement of the gate stacks specific to TFETs with type-II hetero-structures is to realize thin EOT and low MOS interface defect density for two different materials of the source and the channel such as $\mathrm{Ge} / \mathrm{Si}$ [19] and GaAsSb/InGaAs [228-232] by employing the same gate stack formation process, which is not trivial under the different MOS interface physics and chemistry of the two semiconductors. Also, the requirement of low MOS interface defect density could be more stringent in TFETs than in MOSFETs, because MOS interface defects can degrade 
S.S. and $I_{\text {off }}$ through two mechanisms of the reduction of gate potential control over MOS surface potential as well as the increase in TAT and SRH currents via the interface defects.

The third engineering is related to source p-n junction formation, which has peculiar requirements in p-n junction type TFETs. Here, the high steepness of the source impurity profile with low defect density around p-n junctions are strongly required in terms of the thin tunneling distance and low SRH current, which significantly contribute to high $I_{o n}$ and low $I_{o f f}$, respectively. Finally, the drain junction engineering is also important for suppressing ambipolar current and reducing Ioff. When gate potential is biased to form the accumulation region near MOS interfaces, the drain/channel junctions can induce tunneling current as the ambipolar current, which limits the $I_{\text {off }}$ level. Thus, it is necessary to minimize this ambipolar current for suppressing $I_{\text {off }}$ and increasing $I_{\text {on }} / I_{\text {off }}$ ratio through any optimization of the drain structure and/or graded profiles of drain impurities.

In this paper, we emphasize the channel material engineering and the source junction engineering. For the channel material engineering, we have examined TFETs using an $\mathrm{In}_{0.53} \mathrm{Ga} 0.47 \mathrm{As}$ channel as a low and direct band gap material $[14,233,234]$, and TFETs using a combination of a Ge source and a strained-Si channel [14, 235, 236] as a type-II hetero-junction TFET in order to enhance $I_{o n}$. We have realized these devices as the planar structure, which is still effective in easy fabrication under standard CMOS processes and integration with conventional Si CMOS. We have developed novel source junction engineering for InGaAs TFETs. Diffusion of solid phase $\mathrm{Zn}$ diffusion into InGaAs is introduced for realizing source junctions with steep Zn profiles and low defect density. 


\section{3-2 InGaAs TFETs}

InGaAs can be regarded as one of promising materials of TFETs, because of the high tunneling probability due to the narrow and direct bandgap, and recent progress in InGaAs MOSFET technologies. Here, formation of the source junctions with defect-free and steep impurity profiles is mandatory for InGaAs TFETs. In general, it would not be too easy to form such defect-less p-n junctions with steep impurity profiles by ion implantation, because of the difficulty in complete re-crystallization of ion-implanted III-V semiconductors by thermal annealing without significant impurity diffusion and defect generation. From the viewpoint of these requirements, we have introduced solid-phase $\mathrm{Zn}$ diffusion in InGaAs because of the extremely-steep profiles coming from the inherent diffusion property of $\mathrm{Zn}$ in InGaAs and the defect-less process [233, $234,237,238]$.

It is known that $\mathrm{Zn}$ diffuses in InGaAs as interstitials. Here, the following balance equation holds [238].

$$
[\mathrm{Zn}]^{-}+2 h \leftrightarrow\left[\mathrm{Zn}_{\text {int }}\right]^{+}+[\text {III vacancy }]
$$

where $[\mathrm{Zn}], h,\left[\mathrm{Zn}_{\text {int }}\right]$ and [III vacancy] are substitutional $\mathrm{Zn}$ concentration, hole concentration, interstitial $\mathrm{Zn}$ concentration and column-III-element-vacancy concentration in InGaAs, respectively. As a result, the diffusion constant of $\mathrm{Zn}$ is in proportion to $[\mathrm{Zn}]^{2}$. In order to examine the effect of the $[\mathrm{Zn}]$ dependence of the diffusion constant on the profile, the $\mathrm{Zn}$ diffusion profiles are simulated for three types of hypothetical diffusion coefficients, which are assumed to be constant, proportional to $[\mathrm{Zn}]$ and proportional to $[\mathrm{Zn}]^{2}$ ones for simplicity. 
Fig. 23 shows the calculated $\mathrm{Zn}$ profiles under the three diffusion constant models. It is found that the diffusion coefficient proportional to $[\mathrm{Zn}]^{2}$ yields the steepest $\mathrm{Zn}$ profile. This abrupt change of the $\mathrm{Zn}$ concentration can be easily understood by the sharp decrease of the diffusion coefficient proportional to $[\mathrm{Zn}]^{2}$ with decreasing $[\mathrm{Zn}]$, leading to the box-like $\mathrm{Zn}$ profile under this diffusion constant. These simulation results strongly suggest that InGaAs $\mathrm{p}^{+}-\mathrm{n}$ junctions formed by $\mathrm{Zn}$ diffusion have an inherently steep $\mathrm{Zn}$ profile. In addition, solid phase diffusion by using Zn-doped spin-on-glass (SOG) films is expected to provide p-n junctions with a low defect density, because of the damage-less nature of this doping process in comparison with ion implantation. As a result, the $\mathrm{Zn}$ solid phase diffusion by SOG is promising for the formation of source junctions in InGaAs TFETs.

Zn diffusion from the Zn-doped SOG was driven by rapid thermal annealing (RTA) at $500{ }^{\circ} \mathrm{C}$ for 1 minute in a $\mathrm{N}_{2}$ ambient $[233,234]$. Be and $\mathrm{Zn}$ ion implantation was also carried for comparison [239]. Fig. 24(a) shows the Secondary Ion Mass Spectrometry (SIMS) profiles of diffused $\mathrm{Zn}$ and implanted Be atoms. It is found that the steepness of the $\mathrm{Zn}$ profile is $3.5 \mathrm{~nm} / \mathrm{dec}$, which is much steeper than that of the Be profile formed by Be implantation with the energy of $2 \mathrm{keV}$ and the dose of $1 \times 10^{15} \mathrm{~cm}^{-2}, 36 \mathrm{~nm} / \mathrm{dec}$. This result is consistent with the profile calculated using the model that the diffusion constant of $\mathrm{Zn}$ in InGaAs is proportional to the square of the $\mathrm{Zn}$ concentration, also shown in Fig. 24(a), confirming that the steep impurity profile can be automatically obtained by solid phase $\mathrm{Zn}$ diffusion. Actually, the real steepness can be less than 3.5 $\mathrm{nm} / \mathrm{dec}$, which could be determined by atom mixing during the SIMS analysis, as expected in the simulation result.

Fig. 24(b) shows the $I-V$ characteristics of Zn-diffused, Zn-implanted and 
Be-implanted $\operatorname{In}_{0.53} \mathrm{Ga}_{0.47} \mathrm{As} \mathrm{p}^{+}-\mathrm{n}$ junctions. Here, Be ion implantation was carried with the energy of $10 \mathrm{keV}$ and the dose of $1 \times 10^{15} \mathrm{~cm}^{-2}$, and $\mathrm{Zn}$ ion implantation was carried with the energy of $25 \mathrm{keV}$ and the dose of $2 \times 10^{14} \mathrm{~cm}^{-2}$. The activation annealing for Be and $\mathrm{Zn}$ ion implantation was carried by RTA at $600{ }^{\circ} \mathrm{C}$ and $650{ }^{\circ} \mathrm{C}$, respectively, for 1 minute. The Zn-diffused and Be-implanted junctions show the lower ideal factor of 1.2, while the Zn-implanted diode shows the higher ideal factor of 1.7. Also, the minimum current under the reverse bias condition is lowest for the Zn-diffused junctions. These results mean that the $\mathrm{Zn}$ diffusion introduces fewer defects than ion implantation for $\mathrm{p}-\mathrm{n}$ junction formation.

Planar-type $\operatorname{In}_{0.53} \mathrm{Ga}_{0.47} \mathrm{As}$ n-channel TFETs with source regions formed by the present $\mathrm{Zn}$ diffusion process were fabricated on semi-insulating InP substrates [233, 234]. The device structure is shown in the inset of Fig. 25(a). A Ta/ALD $\mathrm{Al}_{2} \mathrm{O}_{3}(3 \mathrm{~nm})$ gate stack and a Ni-InGaAs metal-drain were employed. Fig. 25(a) and (b) show the $I_{D}-V_{G}$ characteristics at $V_{D}$ of $150 \mathrm{mV}$ and the $I_{D^{-}} V_{D}$ characteristics, respectively, of the In $0.53 \mathrm{Ga} 0.47 \mathrm{As}$ TFETs with source regions formed by $\mathrm{Zn}$ diffusion at $500{ }^{\circ} \mathrm{C}$ for $1 \mathrm{~min}$. It is found in Fig. 25(a) that the $\operatorname{In}_{0.53} \mathrm{Ga}_{0.47} \mathrm{As}$ TFETs exhibit the small minimum S.S. of $64 \mathrm{mV} / \mathrm{dec}$. and the high $I_{\text {on }} / I_{\text {off }}$ ratio of $\sim 2 \times 10^{6}$ at the same time as the planar-type III-V TFETs with EOT of $1.3 \mathrm{~nm}$. Also, the good drain current saturation is clearly observed in Fig. 25(b). The comparatively low S.S. values and high $I_{o n}$ are also attributable to narrow tunneling distance in the source $\mathrm{p}-\mathrm{n}$ junctions with the steep $\mathrm{Zn}$ profile and resulting high tunneling probability. Also, the comparatively low $I_{\text {off }}$ and high $I_{\text {on }} / I_{\text {off }}$ are attributable to low source junction leakage current coming from the defect-less p-n junctions formed by $\mathrm{Zn}$ diffusion process. Actually, similar promising electrical characteristics of InGaAs TFETs with Zn-diffusion sources have recently been 
confirmed by another research group [240].

Actually, the low leakage current in the present p-n junctions due to SRH and/or TAT is evident in the temperature dependence of the $I_{d}-V_{g}$ characteristics. Fig. 26 shows the temperature dependence of $I_{d}-\left(V_{g}-V_{t h}\right)$ characteristics at $V_{D}$ of $150 \mathrm{mV}$ of an InGaAs TFET with the source region formed by $\mathrm{Zn}$ diffusion at $500{ }^{\circ} \mathrm{C}$ for $1 \mathrm{~min}$ from $6 \mathrm{~K}$ to $292 \mathrm{~K}$. Here, $V_{t h}$ was defined as $V_{g}$ at $I_{d}$ of $10^{-7} \mathrm{~A} / \mu \mathrm{m}$. Measurement temperatures were varied from $6 \mathrm{~K}, 50 \mathrm{~K}, 171 \mathrm{~K}, 227 \mathrm{~K}, 254 \mathrm{~K}$ to $292 \mathrm{~K}$. Almost no temperature dependence was observed in the subthreshold region, which means that S.S. is almost free from trap-related SRH and TAT currents. This point is quite important, because many previous works on III-V TFETs have reported that the S.S. values have strong temperature dependence and, thus, have been severely degraded by the existence of defects and traps included in source p-n junctions and MOS interfaces. As a consequence, the result of the temperature dependence also supports the fact that the superior $\mathrm{p}^{+}-\mathrm{n}$ source junctions suitable for TFETs can be formed by $\mathrm{Zn}$ solid phase diffusion in InGaAs.

In addition, the results of Fig. 26 indicate that band-to-band tunneling dominates the current of the present TFETs. We have observed the negative differential resistance under the negative drain bias, which is another evidence of the dominance of tunneling mechanism on the carrier transport. Actually, such a weaker temperature dependence of the drain current can be one of possible advantages of TFETs, particularly for applications requiring device operation under a wider temperature range, in comparison with conventional MOSFETs.

\section{3-3 Ge/strained-SOI TFETs}


As emphasized in the section 3-1, the source-channel structures composed of type-II staggered hetero-interfaces, shown in Fig. 22, are quite effective in enhancing the performance of TFETs. Thus, several TFETs using type-II hetero-interfaces such as $\mathrm{In}_{\mathrm{x}} \mathrm{Ga}_{1-\mathrm{x}} \mathrm{As} / \mathrm{GaAsy}_{\mathrm{S}} \mathrm{Sb}_{1-\mathrm{y}}$ [228-232] and $\mathrm{InAs} / \mathrm{Si}[20,241,242]$ have been intensively studied. We have recently proposed a novel type-II hetero-interface TFETs using Ge/tensily-strained Si interfaces [235, 236]. Fig. 27(a) shows the schematic band diagram of the hetero-interface of Ge and tensile strain Si. The higher valence band edge $(\mathrm{Ev})$ of the Ge-source and the lower conduction band edge $\left(\mathrm{E}_{\mathrm{C}}\right)$ of tensile strain Si result in reduction in $E_{g e f f}$, leading to the increase the tunneling probability and $I_{o n}$ and the reduction in S.S. with maintaining the relatively large $\mathrm{E}_{\mathrm{g}}$ of strained-Si in the drain regions, which can suppress the ambipolar leakage current. It has already been reported that pure Ge sources grown on Si have provided Si TFETs with minimum S.S. lower than $60 \mathrm{mV} / \mathrm{dec}$. Also, tensily-strained Si has been regarded as one of the promising materials for TFET channels, because of the smaller bandgap. Actually, tensily-strained Si TFETs with SiGe sources [243, 244] or NW structures [18, 245] have already been reported. Thus, we have combined the Ge source and strained-SOI channels in order to realize the type-II hetero-structure TFETs.

The Ge/strained Si TFET structure, fabricated in this study, is shown in Fig. 27(b) $[235,236]$. In order to examine the impact of tensile strain on the TFET performance, unstrained SOI and two types of strained SOI substrates with 0.8 and $1.1 \%$ biaxial tensile strain were employed. In-situ boron-doped Ge layers were grown at $200{ }^{\circ} \mathrm{C}$ on the source regions of the substrates by MBE. Here, low temperature in-situ doping can lead to steep boron profiles with high boron concentration of as high as $1.3 \times 10^{20} \mathrm{~cm}^{-3}$. A 3-nm-thick $\mathrm{Al}_{2} \mathrm{O}_{3} / \mathrm{GeO}_{\mathrm{x}} / \mathrm{Ge}$ gate stack with EOT of $2.5 \mathrm{~nm}$ was formed by ALD 
combined with ECR plasma post oxidation in order to realize the high quality MOS interfaces between $\mathrm{Al}_{2} \mathrm{O}_{3}$ and $\mathrm{Ge}[8,9]$, as described in the section 2-2-2. Ta was deposited as the gate metal, followed by $\mathrm{Ni}$ and $\mathrm{Al}$ deposition for the source contact and the contact pad, respectively. It was found that one of the key processes is PMA at 400 ${ }^{\circ} \mathrm{C}$ in $\mathrm{N}_{2}$ for 30 minutes.

Fig. 28 shows the $I_{d-} V_{d}$ characteristics of Ge-source TFETs fabricated on un-strained SOI, 0.8 and $1.1 \%$ strained SOI substrates. It has been found that an increase in strain leads to the increase in $I_{o n}$. It has also be observed that gate current and resulting $I_{o f f}$ decrease with an increase in the amount of tensile strain, because of the increase in the barrier height in $E_{C}$ between insulators and strained Si [246], resulting in higher $I_{o n} / I_{o f f}$ with increasing the amount of tensile strain. Here, we have observed that PMA temperature strongly affects the electrical properties of TFETs. PMA at $400{ }^{\circ} \mathrm{C}$ can maximize the $I_{\text {on }} / I_{\text {off }}$ ratio, which amounts to $4.4,2.2$ and $3.7 \times 10^{7}$ for the unstrained, 0.8 and $1.1 \%$ strained SOI TFETs, respectively, and can significantly reduce the S.S. values, which amount to 55,49 and $29 \mathrm{mV} / \mathrm{dec}$. as the minimum value at room temperature for unstrained, 0.8 and $1.1 \%$ strained SOI TFETs, respectively. This improvement in the TFET performance with increasing PMA temperature is attributed to reduction in $D_{i t}$ at the Si MOS interfaces, while $D_{i t}$ at the Ge MOS interfaces does not change with PMA [247]. These results mean that the combination of plasma post oxidation through $\mathrm{Al}_{2} \mathrm{O}_{3}$ and optimized PMA can simultaneously realize the appropriate MOS interface properties for both the Ge source and the strained-Si channel.

Fig. 29 shows a benchmark of the present n-channel TFET performance [233, 234, $236]$ in terms of $I_{\text {on }} / I_{\text {off }}$ ratio versus the minimum S.S. value with other reported ones $[18-20,243,248-254]$ with a variety of channel materials. It is found that the high $I_{o n} / I_{\text {off }}$ 
ratio under the low minimum S.S. can be obtained for the present InGaAs and Ge/strained SOI n-channel TFETs. Further improved performance of these TFETs can be expected by employing thinner EOT gate stacks, optimized drain junctions and multi-gate/NW channel architectures. As a consequence, the InGaAs and Ge source/strained-Si TFETs can be regarded as ones of the superior TFET structures for realizing low $I_{o f f}$ and high $I_{o n}$ at the same time.

\section{CONCLUSIONS}

We have addressed critical issues and challenges for enhancing the performance of Ge/III-V MOSFETs and TFETs, which are strongly expected as devices for realizing ultralow power integrated systems. In order to solve these problems, viable technologies have been presented in this paper. For realizing high performance $\mathrm{Ge} \mathrm{CMOS}, \mathrm{GeO}_{\mathrm{x}} \mathrm{IL}$ formation by plasma post oxidation and atomic D annealing were developed. $\mathrm{HfO}_{2} / \mathrm{Al}_{2} \mathrm{O}_{3} / \mathrm{GeO}_{x} / \mathrm{Ge}$ gate stacks formed by this method have realized high performance Ge n- and p-MOSFETs with EOT of $0.76 \mathrm{~nm}$ having $D_{i t}$ of $\sim 3 \times 10^{11}$ $\mathrm{cm}^{-2} \mathrm{eV}^{-1}$, whose peak electron and hole mobility amount to 689 and $546 \mathrm{~cm}^{2} / \mathrm{Vs}$, respectively. The InGaAs/InAs/InGaAs-OI QW channels combined with Tri-gate structures have been introduced for the technologies enhancing the performance of III-V n-MOSFETs. The InGaAs/InAs/InGaAs-OI QW Tri-gate n-MOSFETs with the Fin width of $\sim 40 \mathrm{~nm}$ have offered $I_{o n}$ of $\sim 380 \mu \mathrm{A} / \mu \mathrm{m}$ at $V_{d}$ and $V_{g}$ of $0.5 \mathrm{~V}$ under $I_{\text {off }}$ of 100 $\mathrm{nA} / \mu \mathrm{m}$. A fabrication technique of the InGaAs ultrathin body channels on large size $\mathrm{Si}$ wafers has also been demonstrated. Also, the effectiveness of Ge/III-V materials on TFETs through the enhancement of tunneling probability has been demonstrated. Superior junction formation and MOS interface control technologies are key factors to 
realize TFETs using Ge/III-V. The $\mathrm{p}^{+}$-n junction formation in InGaAs using $\mathrm{Zn}$ diffusion has realized excellent planar In 0.53 Ga0.47As $n$-TFET characteristics, because of the defect-less source junctions with steep Zn profiles. Ge/tensily-strained Si n-TFETs with the type-II hetero-interfaces have also been found to exhibit high $I_{o n} / I_{\text {off }}$ ratio and low minimum S.S., because of reduction in effective $E_{g}$, steep B profiles by in-situ B doping in Ge and improved MOS interface properties with both Ge and strained Si.

\section{Acknowledgments}

This work was partly supported by JST-CREST, R\&D Program for Innovative Energy Efficiency Technology from NEDO, a Grant-in-Aid for Scientific Research (26249038) from MEXT. The authors would like to thank Drs. J. Li and Y.-C. Kao in IntelliEPI, Inc., Drs. H. Yamada, O. Ichikawa, T. Osada and M. Hata in Sumitomo Chemical Corporation, and Drs. H. Yokoyama and M. Mitsuhara in NTT for their collaborations. 


\section{REFERENCES}

[1] T. Sakurai, Perspectives of power-aware electronics (Plenary), In: Proc. ISSCC Dig. Tech. Papers.; 2003. p. 26-29.

[2] M. Lundstrom and Z. Ren, Essential physics of carrier transport in nanoscale MOSFETs, IEEE Trans. Electron Devices 2002;49:133-141.

[3] M. Lundstrom and J. Guo, Nanoscale Transistors. New York: Springer-Verlag, 2006.

[4] K. Natori, Ballistic metal-oxide-semiconductor field effect transistor, J. Appl. Phys. 1994;76:4879-4890.

[5] K. Natori, Scaling limit of the MOS transistor-A ballistic MOSFET, IEICE Trans. Electron. 2001;E84-C:1029-1036.

[6] S. Takagi, Re-examination of subband structure engineering in ultrashort channel MOSFETs under ballistic transport regime, In: VLSI Symp. Tech. Dig. 2003. p. $115-116$.

[7] S. Takagi, T. Irisawa, T. Tezuka, T. Numata, S. Nakaharai, N. Hirashita, Y. Moriyama, K. Usuda, E. Toyoda, S. Dissanayake, M. Shichijo, R. Nakane, S. Sugahara, M. Takenaka, and N. Sugiyama, Carrier-Transport-Enhanced Channel CMOS for Improved Power Consumption and Performance, IEEE Trans. Electron Devices 2008;55:21-39.

[8] S. Takagi, R. Zhang, S.-H Kim, N. Taoka, M. Yokoyama, J.-K. Suh, R. Suzuki, and M. Takenaka, MOS interface and channel engineering for high-mobility Ge/III-V CMOS, In: IEDM Tech. Dig. 2012. p. 505-508.

[9] S. Takagi, S.-H. Kim, M. Yokoyama, R. Zhang, N. Taoka, Y. Urabe, T. Yasuda, H. Yamada, O. Ichikawa, N. Fukuhara, M. Hata and M. Takenaka, High Mobility CMOS 
Technologies using III-V/Ge Channels on Si platform, Solid State Electronics 2013; $88: 2-8$.

[10] S. Takagi, R. Zhang, J. Suh, S.-H. Kim, M. Yokoyama, K. Nishi and M. Takenaka, III-V/Ge Channel MOS Device Technologies in Nano CMOS era, Jpn. J. Appl. Phys. 2015;54:06FA01.

[11] A. C. Seabaugh and Q. Zhang, Low-Voltage Tunnel Transistors for Beyond CMOS Logic, IEEE Proc. 2010;98:2095.

[12] A. M. Ionescu and H. Riel, Tunnel field-effect transistors as energy-efficient electronic switches, Nature, 2011;479:329.

[13] H. Lu and A. C. Seabaugh, Tunnel Field-Effect Transistors: State-of-the-Art, IEEE J. Electron Device Society 2014;2:44.

[14] S. Takagi, M.-S. Kim, M. Noguchi, S.-M. Ji, K. Nishi and M. Takenaka, III-V and Ge/strained SOI Tunneling FET Technologies for Low Power LSIs, In: IEEE Symp. on VLSI Technol. 2015; T22-T23.

[15] K. Jeon et al., Si tunnel transistors with a novel silicided source and $46 \mathrm{mV} / \mathrm{dec}$ swing, In: Proc. VLSI Symp. Tech. Dig., 2010, p. 121-122.

[16] R. Gandhi, Z. Chen, N. Singh, K. Banerjee, and S. Lee, Vertical Si nanowire n-type tunneling FETs with low subthreshold swing at room temperature, IEEE Electron Device Lett. 2011;32:437-439.

[17] R. Gandhi, Z. Chen, N. Singh, K. Banerjee, and S. Lee, CMOS-compatible vertical-silicon-nanowire gate-all-around $\mathrm{p}$ type tunneling FETs with $\leq 50$-mV/decade subthreshold swing, IEEE Electron Device Lett. 2011;32:1504-1506.

[18] L. Knoll, Q. T. Zhao, A. Nichau, S.Trellenkamp, S. Richter, A. Schäfer, D. Esseni, L. Selmi, K. K. Bourdelle, and S. Mantl, Inverters with strained Si nanowire 
complementary tunnel field-effect transistors, IEEE Electron Device Lett. $2013 ; 34: 813-815$.

[19] S. H. Kim, H. Kam, C. Hu, and T.-J. K. Liu, Germanium-source tunnel field effect transistors with record high ION/IOFF, In: Proc. VLSI Symp. Tech. Dig. 2009; p. $178-179$.

[20] K. Tomioka, M. Yoshimura, and T. Fukui, Steep-slope tunnel field effect transistors using III-V nanowire/Si heterojunction, In: Proc. VLSI Symp. Tech. Dig. 2012; p. $47-48$.

[21] B. Ganjipour, J. Wallentin, M. T. Borgström, L. Samuelson, and C. Thelander, Tunnel field-effect transistors based on InP-GaAs heterostructure nanowires, ACS Nano 2012;6:3109-3113.

[22] D. Sarkar, X. Xie, W. Liu, W. Cao, J. Kang, Y. Gong, S. Kraemer, P. M. Ajayan and K. Banerjee, A subthermionic tunnel field-effect transistor with an atomically thin channel, Nature 2015;526:91-95.

[23] J. A. del Alamo, Nanometre-scale electronics with III-V compound semiconductors, Nature 2011;479:317-323.

[24] M. Yokoyama, R. Iida, S.-H. Kim, N. Taoka, Y. Urabe, H. Takagi, T. Yasuda, H. Yamada, N. Fukuhara, M. Hata, M. Sugiyama, Y. Nakano, M. Takenaka and S. Takagi, Sub-10-nm extremely-thin body InGaAs-on-insulator MOSFETs on Si wafers with ultrathin $\mathrm{Al}_{2} \mathrm{O}_{3}$ buried oxide layers, IEEE Electron Device Lett. 2011;32:1218-1220

[25] M. Radosavljevic, G. Dewey, D. Basu, J. Boardman, B. Chu-Kung, J. M. Fastenau, S. Kabehie, J. Kavalieros, V. Le, W. K. Liu, D. Lubyshev, M. Metz, K. Millard, N. Mukherjee, L. Pan, R. Pillarisetty, W. Rachmady, U. Shah, H. W. Then, and R. Chau, Electrostatics Improvement in 3-D Tri-gate Over Ultra-Thin Body Planar InGaAs 
Quantum Well Field Effect Transistors with High-K Gate Dielectric and Scaled Gate-to-Drain/Gate-to-Source Separation, In: IEDM Tech. Dig. 2011; p. 765-768.

[26] J. J. Gu, X. W. Wang, H. Wu, J. Shao, A. T. Neal, M. J. Manfra, R. G. Gordon, and P. D. Ye, 20-80nm Channel Length InGaAs Gate-all-around Nanowire MOSFETs with EOT $=1.2 \mathrm{~nm}$ and Lowest $\mathrm{SS}=63 \mathrm{mV} / \mathrm{dec}$, In: IEDM Tech. Dig. 2012; p. 633-636.

[27] D.-H. Kim, P. Hundal, A. Papavasiliou, P. Chen, C. King, J. Paniagua, M. Urteaga, B. Brar, Y. G. Kim, J.-M. Kuo, J. Li, P. Pinsukanjana, and Y. C. Kao, E-mode Planar Lg $=35 \mathrm{~nm}$ In0.7Ga0.3As MOSFETs with $\mathrm{InP} / \mathrm{Al}_{2} \mathrm{O}_{3} / \mathrm{HfO}_{2}(\mathrm{EOT}=0.8 \mathrm{~nm})$ Composite Insulator, In: IEDM Tech. Dig. 2012; p. 761-764.

[28] T.-W. Kim, D.-H Kim, D.-H. Koh, R. J. W. Hill, R. T. P. Lee, M.H Wong, T. Cunningham, J. A. del Alamo, S. K. Banerjee, S. Oktyabrsky, A. Greene, Y. Ohsawa, Y. Trickett, G Nakamura, Q. Li, K.M. Lau, C. Hobbs, P. D. Kirsch and R. Jammy, ETB-QW InAs MOSFET with scaled body for Improved Electrostatics, In: IEDM Tech. Dig. 2011; p. 765-768.

[29] S. Lee, C.-Y. Huang, A. D. Carter, D. C. Elias, J. J. M. Law, V. Chobpattana, S. Krämer, B. J. Thibeault, W. Mitchell, S. Stemmer, A. C. Gossard, and M. J. W. Rodwell, Record Extrinsic Transconductance $\left(2.45 \mathrm{mS} / \mu \mathrm{m}\right.$ at $\left.\mathrm{V}_{\mathrm{DS}}=0.5 \mathrm{~V}\right) \mathrm{InAs} / \mathrm{In}_{0.53} \mathrm{Ga}_{0.47} \mathrm{As}$ Channel MOSFETs Using MOCVD Source-Drain Regrowth, In: Symp. VLSI Tech. Dig. 2013; T246-T247.

[30] S.-H. Kim, M. Yokoyama, N. Taoka, R. Nakane, T. Yasuda, O. Ichikawa, N. Fukuhara, M. Hata, M. Takenaka and S. Takagi, Sub-60 nm Extremely-thin Body InxGa1-xAs-On-Insulator MOSFETs on $\mathrm{Si}$ with Ni-InGaAs Metal $\mathrm{S} / \mathrm{D}$ and MOS Interface Buffer Engineering and its scalability, IEEE Trans. Electron Devices 2013;60; $2512-2517$ 
[31] S.-H. Kim, M. Yokoyama, R. Nakane, O. Ichikawa, T. Osada, M. Hata, M. Takenaka and S. Takagi, High Performance InAs-On-Insulator nMOSFETs with Ni-InGaAs S/D Realized by Contact Resistance Reduction Technology, IEEE Trans. Electron Devices 2013;60:3342-3350.

[32] J. A. del Alamo, D. Antoniadis, A. Guo, D.-H. Kim, T.-W. Kim, J. Lin, W. Lu, A. Vardi, and X. Zhao, InGaAs MOSFETs for CMOS: Recent Advances in Process Technology, IEDM Tech. Dig. 2013; p. 24-27.

[33] R. T. P. Lee, R. J. W. Hill, W.-Y. Loh, R.-H. Baek, S. Deora, K. Matthews, C. Huffman, K. Majumdar, T. Michalak, C. Borst, P. Y. Hung, C.-H. Chen, J.-H. Yum, T.-W. Kim, C.Y. Kang, W.-E. Wang, D.-H. Kim, C. Hobbs, and P. D. Kirsch, VLSI Processed InGaAs on Si MOSFETs with Thermally Stable, Self-Aligned Ni-InGaAs Contacts Achieving: Enhanced Drive Current and Pathway Towards a Unified Contact Module, IEDM Tech. Dig. 2013; p. 44-47.

[34] X. Zhou, Q. Li, C. W. Tang, and K. M. Lau, 30nm Enhancement-mode In0.53Ga0.47As MOSFETs on Si Substrates Grown by MOCVD Exhibiting High Transconductance and Low On-resistance, In: IEDM Tech. Dig. 2012, p. 773-777.

[35] S. W. Chang, X. Li, R. Oxland, S. W. Wang, C. H. Wang, R. Contreras-Guerrero, K. K. Bhuwalka, G. Doornbos, T. Vasen, M. C. Holland, G. Vellianitis, M. J. H. van Dal, B. Duriez, M. Edirisooriya, J. S Rojas-Ramirez, P. Ramvall, S. Thoms, U. Peralagu, C. H. Hsieh, Y. S. Chang, K. M. Yin, E. Lind, L.-E. Wernersson, R. Droopad, I. Thayne, M. Passlack, and C. H. Diaz, InAs N-MOSFETs with record performance of $\mathrm{I}_{\mathrm{on}}=600$ $\mu \mathrm{A} / \mu \mathrm{m}$ at $\mathrm{I}_{\text {off }}=100 \mathrm{nA} / \mu \mathrm{m}\left(\mathrm{V}_{\mathrm{d}}=0.5 \mathrm{~V}\right)$, In: IEDM Tech. Dig. 2013; p. 417-420.

[36] J. Lin, X. Zhao, T. Yu, D. A. Antoniadis, and J. A. del Alamo, A New Self-aligned Quantum-Well MOSFET Architecture Fabricated by a Scalable Tight-Pitch Process, In: 
IEDM Tech. Dig. 2013; p. 421-424.

[37] T.-W. Kim, D.-H. Kim, D.H. Koh1, H.M. Kwon, R.H. Baek, D. Veksler, C. Huffman, K. Matthews, S. Oktyabrsky, A. Greene, Y. Ohsawa, A. Ko, H. Nakajima, M. Takahashi, T. Nishizuka, H. Ohtake, S. K. Banerjee, S.H. Shin, D.-H. Ko, C. Kang, D. Gilmer, R.J.W. Hill, W. Maszara, C. Hobbs and P. D. Kirsch, Sub-100 nm InGaAs Quantum-Well (QW) Tri-Gate MOSFETs with $\mathrm{Al}_{2} \mathrm{O}_{3} / \mathrm{HfO}_{2} \quad(\mathrm{EOT}<1 \mathrm{~nm})$ for Low-Power Logic Applications, In: IEDM Tech. Dig. 2013; p. 425-428.

[38] S.-H. Kim, M. Yokoyama, R. Nakane, M. Ichikawa, T. Osada, M. Hata, M. Takenaka, High Performance Sub-20-nm-Channel-Length Extremely-Thin Body InAs-on-Insulator Tri-Gate MOSFETs with High Short Channel Effect Immunity and $V_{\text {th }}$ Tunability, In: IEDM Tech. Dig. 2013; p. 429-432.

[39] S.-H. Kim, M. Yokoyama, R. Nakane, O. Ichikawa, T. Osada, M. Hata, M. Takenaka and S. Takagi, High Performance Tri-gate Extremely-thin-Body InAs-on-Insulator MOSFETs with high short channel effect immunity and Vth tenability, IEEE Trans. Electron Devices 2014;61:1354-1360.

[40] C.-S. Shin, W.-K. Park, SH. Shin, Y. D. Cho, D. H. Ko, T.-W. Kim, D. H. Koh, H. M. Kwon, R. J. W. Hill, P. Kirsch, W. Maszara and D.-H. Kim, Sub-100 nm Regrown S/D Gate-Last In $0.7 \mathrm{Ga} 0.3$ As QW MOSFETs with $\mu_{\mathrm{n}, \text { eff }}>5,500 \mathrm{~cm}^{2} / \mathrm{V}$-s, In: Symp. VLSI Tech. Dig. 2014, p. T30-T31.

[41] N. Waldron, C. Merckling, W. Guo, P. Ong, L. Teugels, S. Ansar, D. Tsvetanova, F. Sebaai, D. H. van Dorp, A. Milenin, D. Lin, L. Nyns, J. Mitard, A. Pourghaderi, B. Douhard, O. Richard, H. Bender, G. Boccardi, M. Caymax, M. Heyns, W. Vandervorst, K. Barla, N. Collaert, and A.V-Y. Thean, An InGaAs/InP Quantum Well FinFet Using the Replacement Fin Process Integrated in an RMG Flow on 300mm Si Substrates, In: 
Symp. VLSI Tech. Dig. 2014; p. T32-T33.

[42] S. Lee, V. Chobpattana, C.-Y. Huang, B. J. Thibeault, W. Mitchell, S. Stemmer, A. C. Gossard, and M. J. W. Rodwell, Record Ion $\left(0.50 \mathrm{~mA} / \mu \mathrm{m}\right.$ at $\mathrm{V}_{\mathrm{DD}}=0.5 \mathrm{~V}$ and $\mathrm{I}_{\text {off }}=$ $100 \mathrm{nA} / \mu \mathrm{m}) 25 \mathrm{~nm}$-Gate-Length $\mathrm{ZrO}_{2} / \mathrm{InAs} / \mathrm{InAlAs}$ MOSFETs, In: Symp. VLSI Tech. Dig. 2014, p. T54-T55.

[43] C. Y. Huang, S. Lee, V. Chobpattana, S. Stemmer, A. C. Gossard, B. Thibeault, W. Mitchell and M. Rodwell, Low Power III-V InGaAs MOSFETs Featuring InP Recessed Source/Drain Spacers with Ion $=120 \mu \mathrm{A} / \mu \mathrm{m}$ at $\mathrm{I}_{\text {off }}=1 \mathrm{nA} / \mu \mathrm{m}$ and $\mathrm{V}_{\mathrm{DS}}=0.5 \mathrm{~V}$, In: IEDM Tech. Dig. 2014; p. 586-589.

[44] V. Djara, V. Deshpande, E. Uccelli, N. Daix, D. Caimi, C. Rossel, M. Sousa, H. Siegwart, C. Marchiori, J.M. Hartmann, K.-T. Shiu, C.-W. Weng, M. Krishnan, M. Lofaro, R. Steiner, D. Sadana, D. Lubyshev, A. Liu, L. Czornomaz and J. Fompeyrine, An InGaAs on Si Platform for CMOS with $200 \mathrm{~mm}$ InGaAs-OI Substrate, Gate-first, Replacement Gate Planar and FinFETs Down to $120 \mathrm{~nm}$ Contact Pitch, In: Symp. VLSI Tech. Dig. 2015, p. T176-T177.

[45] M. L. Huang, S. W. Chang, M. K. Chen, C. H. Fan, H. T. Lin, C. H. Lin, R. L. Chu, K. Y. Lee, M. A. Khaderbad, Z. C. Chen, C. H. Lin, C. H. Chen, L. T. Lin, H. J. Lin, H. C. Chang, C. L. Yang, Y. K. Leung, Y.-C. Yeo, S. M. Jang, H. Y. Hwang and C. H. Diaz, In0.53Ga0.47As MOSFETs with high channel mobility and gate stack quality fabricated on 300 mm Si substrate, In: Symp. VLSI Tech. Dig. 2015, p. T204-T205.

[46] N. Waldron, S. Sioncke, J. Franco, L. Nyns, A. Vais1, X. Zhou, H.C. Lin, G.Boccardi, J.W. Maes, Q. Xie, M. Givens, F. Tang, X. Jiang, E. Chiu, A. Opdebeeck, C. Merckling, F. Sebaai, D. van Dorp, L. Teugels, A. S. Hernandez, K. De Meyer, K. Barla, N. Collaert. Y-V. Thean, Gate-All-Around InGaAs Nanowire FETS with Peak 
Transconductance of $2200 \mu \mathrm{S} / \mu \mathrm{m}$ at $50 \mathrm{~nm} \mathrm{Lg}$ using a Replacement Fin RMG Flow, In: IEDM Tech. Dig. 2015; p. 799-802.

[47] M. Berg, K.-M. Persson, O.-P. Kilpi, J. Svensson, E. Lind, and L.-E. Wernersson, Self-Aligned, Gate-Last Process for Vertical InAs Nanowire MOSFETs on Si, In: IEDM Tech. Dig. 2015; p. 803-806.

[48] C. B. Zota, L.-E. Wernersson and E. Lind, Single Suspended InGaAs Nanowire MOSFETs, In: IEDM Tech. Dig. 2015; p. 811-814.

[49] S. Deora, G. Bersuker, W.-Y. Loh, D. Veksler, K. Matthews, T. W. Kim, R. T. P. Lee, R. J. W. Hill, D.-H. Kim, W.-E. Wang, C. Hobbs, and P. D. Kirsch, "Positive Bias Instability and Recovery in InGaAs Channel nMOSFETs", IEEE Trans. Device and Materials Reliability 2013;13:507-514.

[50] J. Franco, B. Kaczer, Ph. Roussel, J. Mitard, S, Sioncke, L. Witters, H. Mertens, T. Grasser, and G. Groeseneken, Understanding the Suppressed Charge Trapping in Relaxed- and Strained-Ge/SiO $2 / \mathrm{HfO}_{2}$ pMOSFETs and Implications for the Screening of Alternative High-Mobility Substrate/Dielectric CMOS Gate Stacks, In: IEDM Tech. Dig. 2013; p. 397-400.

[51] J. Franco, A. Alian, B. Kaczer, D. Lin, T. Ivanov, A. Pourghaderi, K. Martens, Y. Mols, D. Zhou, N. Waldron, S. Sioncke, T. Kauerauf, N. Collaert, A. Thean, M. Heyns, and G. Groeseneken, Suitability of high-k gate oxides for III-V devices: a PBTI study in In $0.53 \mathrm{Ga}_{0.47} \mathrm{As}$ devices with $\mathrm{Al}_{2} \mathrm{O}_{3}$, In: IEEE Proc. International Reliability Physics Symposium (IRPS), 2014, p. 6A2.1-2.6.

[52] G. Groeseneken, J. Franco, M. Cho, B. Kaczer, M. Toledano-Luque, Ph. Roussel, T. Kauerauf, A. Alian, J. Mitard, H. Arimura, D. Lin, N. Waldron, S. Sioncke, L. Witters, H. Mertens, L. Ragnarsson, M. Heyns, N. Collaert, A. Thean and A. Steegen, BTI 
Reliability of Advanced Gate Stacks for Beyond-Silicon Devices: Challenges and Opportunities (Invited), In: IEDM Tech. Dig. 2014; p. 828-831.

[53] J. Nah, H. Fang, C. Wang, K. Takei, M.-H. Lee, E. Plis, S. Krishna, and A. Javey, III-V Complementary Metal-Oxide-Semiconductor Electronics on Silicon Substrates, Nano Lett. 2012;12:3592.

[54] Z. Yuan, A. Nainani, A. Kumar, X. Guan, B. R. Bennett, J. B. Boos, M. G. Ancona, and K. C. Saraswat, InGaSb: Single Channel Solution for Realizing III-V CMOS, In: Symp. VLSI Tech. Dig. 2012; p. 185-186.

[55] Z. Yuan, A. Kumar, C.-Y. Chen, A. Nainani, P. Griffin, A. Wang, W. Wang, and M.-H. Wong, Optimal Device Architecture and Hetero-Integration Scheme for III-V CMOS, In: Symp. VLSI Tech. Dig. 2013; p. T54-T55.

[56] M. Yokoyama, H. Yokoyama, M. Takenaka, and S. Takagi, III-V Single Structure CMOS by Using Ultrathin Body InAs/GaSb-OI Channels on Si, In: Symp. VLSI Tech. Dig. 2014; p. 34-35.

[57] B. Ganjipour, M. Ek, B. M. Borg, K. A. Dick, M.-E. Pistol, L.-E. Wernersson, and C. Thelander, Carrier control and transport modulation in GaSb/InAsSb core/shell nanowires, Appl. Phys. Lett. 2012;101:103501.

[58] A. W. Dey, J. Svensson, B. M. Borg, M. Ek, and L.-E. Wernersson, Single InAs/GaSb Nanowire Low-Power CMOS Inverter, Nano Lett. 2012;12:5593.

[59] M. K. Hudait, G. Dewey, S. Datta, J. M. Fastenau, J. Kavalieros, W. K. Liu, D. Lubyshev, R. Pillarisetty, W. Rachmady, M. Radosavljevic, T. Rakshit and R. Chau, Heterogeneous Integration of Enhancement Mode In0.7Ga0.3As Quantum Well Transistor on Silicon Substrate using Thin $(\leq 2 \mu \mathrm{m})$ Composite Buffer Architecture for High-Speed and Low-voltage ( 0.5V) Logic Applications, In: IEDM Tech. Dig. 2007; p. 625-628. 
[60] S. Datta, G. Dewey, J. M. Fastenau, M. K. Hudait, D. Loubychev, W. K. Liu, M. Radosavljevic, W. Rachmady and R. Chau, Ultrahigh-Speed 0.5 V Supply Voltage In 0.7 Ga0.3As Quantum-Well Transistors on Silicon Substrate, IEEE Electron Device Lett. 2007;28:685.

[61] M. Radosavljevic, B. Chu-Kung, S. Corcoran, G. Dewey, M. K. Hudait, J. M. Fastenau, J. Kavalieros, W. K. Liu, D. Lubyshev, M. Metz, K. Millard, N. Mukherjee, W. Rachmady, U. Shah, and R. Chau, Advanced High-K Gate Dielectric for High-Performance Short-Channel In0.7Ga0.3As Quantum Well Field Effect Transistors on Silicon Substrate for Low Power Logic Applications, In: IEDM Tech. Dig. 2009; p. 319-322.

[62] R. J. W. Hill, C. Park, J. Barnett, J. Price, J. Huang, N. Goel, W.Y. Loh, J. Oh, C. E. Smith, P. Kirsch, P. Majhi, and R. Jammy, Self-aligned III-VMOSFETs heterointegrated on a $200 \mathrm{~mm}$ Si substrate using an industry standard process flow, In: IEDM Tech. Dig. 2010; p. 130-133.

[63] N. Mukherjee, J. Boardman, B. Chu-Kung, G. Dewey, A. Eisenbach, J. Fastenau, J. Kavalieros, W. K. Liu, D. Lubyshev, M. Metz, K. Millard, M. Radosavljevic, T. Stewart, H. W. Then, P. Tolchinsky, and R. Chau, MOVPE III-V Material Growth on Silicon Substrates and its Comparison to MBE for Future High Performance and Low Power Logic Applications (Invited Paper), In: IEDM Tech. Dig. 2011; p. 821-824.

[64] Y. Q. Wu, M. Xu, P. D. Ye, Z. Cheng, J. Li, J.-S. Park, J. Hydrick, J. Bai, M. Carroll, J. G. Fiorenza, and A. Lochtefeld, Atomic-layer-deposited $\mathrm{Al}_{2} \mathrm{O}_{3} / \mathrm{GaAs}$ metal-oxidesemiconductor field-effect transistor on $\mathrm{Si}$ substrate using aspect ratio trapping technique, Appl. Phys. Lett. 2008;93:242106.

[65] T. Hoshii, M. Deura, M. Sugiyama, R. Nakane, S. Sugahara, M. Takenaka, Y. 
Nakano, and S. Takagi, Epitaxial lateral overgrowth of InGaAs on $\mathrm{SiO}_{2}$ from (111) $\mathrm{Si}$ micro channel areas, Physica Status Solidi 2008;C 5:2733.

[66] M. Deura, T. Hoshii, M. Takenaka, S. Takagi, Y. Nakano, and M. Sugiyama, Effect of Ga content on crystal shape in micro-channel selective-area MOVPE of InGaAs on Si, J. Cryst. Growth 2008;310:4768.

[67] G. Wang, M. R. Leys, R. Loo, O. Richard, H. Bender, N. Waldron, G. Brammertz, J. Dekoster, W. Wang, M. Seefeldt, M. Caymax, and M. M. Heyns, Selective area growth of high quality InP on Si (001) substrates, Appl. Phys. Lett. 2010;97:121913.

[68] G. Wang, M. Leys, R. Loo, O. Richard, H. Bender, G. Brammertz, N. Waldron, W.-E Wang, J. Dekoster, M. Caymax, M. Seefeldt, and M. Heyns, Selective Area Growth of InP and Defect Elimination on Si (001) Substrates, J. Electrochem. Soc. 2011;158:H645.

[69] R. Loo, G. Wang, T. Orzali, N. Waldron, C. Merckling, M. R. Leys, O. Richard, H. Bender, P. Eyben, W. Vandervorst, and M. Caymax, Selective area growth of InP on On-Axis Si (001) substrates with low antiphase boundary formation, J. Electrochem. Soc. 2012;159: H260.

[70] C. Merckling, N. Waldron, S. Jiang, W. Guo, N. Collaert, M. Caymax, E. Vancoille, K. Barla, A. Thean, M. Heyns, and W. Vandervorst, Heteroepitaxy of InP on $\mathrm{Si}(001)$ by selective-area metal organic vapor-phase epitaxy in sub-50 nm width trenches: The role of the nucleation layer and the recess engineering, J. Appl. Phys. 2014;115:023710.

[71] N. Waldron, C. Merckling, L. Teugels, P. Ong, S. A. U. Ibrahim, F. Sebaai, A. Pourghaderi, K. Barla, N. Collaert, and A. V.-Y. Thean, InGaAs gate-all-around nanowire devices on 300mm Si substrates, IEEE Electron Device Lett. 2014;35:1097. [72] L. Czornomaz, E. Uccelli, M. Sousa, V. Deshpande, V. Djara, D. Caimi, M. D. 
Rossell, R. Erni and J. Fompeyrine, Confined Epitaxial Lateral Overgrowth (CELO): A Novel Concept for Scalable Integration of CMOS-compatible InGaAs-on-insulator MOSFETs on Large-Area Si Substrates, In: Symp. VLSI Tech. Dig. 2015; p. T172-T173.

[73] M. Yokoyama, T. Yasuda, H. Takagi, H. Yamada, N. Fukuda, M. Hata, M. Sugiyama, Y. Nakano, M. Takenaka, and S. Takagi, High mobility metal S/D III-V-On-Insuator MOSFETs on a Si substrate using direct wafer bonding, In: Symp. VLSI Tech. Dig. 2009; p. 242-243.

[74] M. Yokoyama, T. Yasuda, H. Takagi, H. Yamada, N. Fukuhara, M. Hata, M. Sugiyama, Y. Nakano, M. Takenaka, and S. Takagi, Thin Body III-V-Semiconductor-on-Insulator Metal-Oxide-Semiconductor Field-Effect Transistors on Si Fabricated Using Direct Wafer Bonding, Appl. Phys. Express 2009;2:124501.

[75] M. Yokoyama, T. Yasuda, H. Takagi, N. Miyata, Y. Urabe, H. Ishii, H. Yamada, N. Fukuhara, M. Hata, M. Sugiyama, Y. Nakano, M. Takenaka, and S. Takagi, III-V-semiconductor-on-insulator n-channel metal-insulator-semiconductor field-effect transistors with buried $\mathrm{Al}_{2} \mathrm{O}_{3}$ layers and sulfur passivation: Reduction in carrier scattering at the bottom interface, Appl. Phys. Lett. 2010;96:142106.

[76] M. Yokoyama, Y. Urabe, T. Yasuda, H. Takagi, H. Ishii, N. Miyata, H. Yamada, N. Fukuhara, M. Hata, M. Sugiyama, Y. Nakano, M. Takenaka, and S. Takagi, High Mobility III-V-On-Insulator MOSFETs on $\mathrm{Si}$ with $\mathrm{ALD}-\mathrm{Al}_{2} \mathrm{O}_{3}$ BOX layers, In: Symp. VLSI Tech. Dig. 2010; p. 235-236.

[77] H. Ko, K. Takei, R. Kapadia, S. Chuang, H. Fang, P. W. Leu, K. Ganapathi, E. Plis, H. S. Kim, S.-Y. Chen, M. Madsen, A. C. Ford, Y.-L. Chueh, S. Krishna, S. Salahuddin, and A. Javey, Ultrathin compound semiconductor on insulator layers for 
high-performance nanoscale transistors, Nature 2010;468:286.

[78] H. Takita, N. Hashimoto, C.-T. Nguyen, M. Kudo, M. Akabori, and T. Suzuki, Electron transport properties of InAs ultrathin films obtained by epitaxial lift-off and van der Waals bonding on flexible substrates, Appl. Phys. Lett. 2010;97:012102.

[79] Y. Urabe, M. Yokoyama, H. Takagi, T. Yasuda, N. Miyata, H. Yamada, N. Fukuhara, M. Hata, M. Takenaka, and S. Takagi, III-V-semiconductor-on-insulator n-channel metal-insulator-semiconductor field-effect transistors with buried $\mathrm{Al}_{2} \mathrm{O}_{3}$ layers and sulfur passivation: Reduction in carrier scattering at the bottom interface, Appl. Phys. Lett. 2010;97:253502.

[80] M. Yokoyama, R. Iida, S.-H. Kim, N. Taoka, Y. Urabe, T. Yasuda, H. Takagi, H. Yamada, N. Fukuhara, M. Hata, M. Sugiyama, Y. Nakano, M. Takenaka, and S. Takagi, Extremely-thin-body InGaAs-On-Insulator MOSFETs on Si fabricated by direct wafer bonding, In: IEDM Tech. Dig. 2010; p. 46-49.

[81] M. Yokoyama, T. Yasuda, H. Takagi, H. Yamada, N. Fukuhara, M. Hata, M. Sugiyama, Y. Nakano, M. Takenaka and S. Takagi, Ultrathin Body InGaAs-on-Insulator Metal-Oxide-Semiconductor Field-Effect Transistors with InP Passivation Layers on Si Substrates Fabricated by Direct Wafer Bonding, Appl. Phys. Express 2011;4:054202.

[82] S.-H. Kim, M. Yokoyama, N. Taoka, R. Iida, S. Lee, R. Nakane, Y. Urabe, N. Miyata, T. Yasuda, H. Yamada, N. Fukuhara, M. Hata, M. Takenaka, and S. Takagi, High Performance Extremely-Thin Body III-V-On-Insulator MOSFETs on a $\mathrm{Si}$ Substrate with Ni-InGaAs Metal S/D and MOS Interface Buffer Engineering, In: Symp. VLSI Tech. Dig. 2011; p. 58-59.

[83] M. Yokoyama, S. H. Kim, R. Zhang, N. Taoka, Y. Urabe, T. Maeda, H. Takagi, T. Yasuda, H. Yamada, O. Ichikawa, N. Fukuhara, M. Hata, M. Sugiyama, Y. Nakano, M. 
Takenaka, and S. Takagi, CMOS integration of InGaAs nMOSFETs and Ge pMOSFETs with self-align Ni-based metal S/D using direct wafer bonding, In: Symp. VLSI Tech. Dig. 2011 ; p. 60-61.

[84] S.-H. Kim, M. Yokoyama, N. Taoka, R. Iida, S.-H. Lee, R. Nakane, Y. Urabe, N. Miyata, T. Yasuda, H. Yamada, N. Fukuhara, M. Hata, M. Takenaka, and S. Takagi, High Performance Extremely Thin Body InGaAs-on-Insulator Metal-Oxide-Semiconductor Field-Effect Transistors on Si Substrates with Ni-InGaAs Metal Source/Drain, Appl. Phys. Express 2011;4:114201.

[85] K. Takei, S. Chuang, H. Fang, R. Kapadia, C.-H. Liu, J. Nah, H. S. Kim, E. Plis, S. Krishna, Y.-L. Chueh, and A. Javey, Benchmarking the performance of ultrathin body InAs-on-insulator transistors as a function of body thickness, Appl. Phys. Lett. 2011;99:103507.

[86] K. Takei, H. Fang, S. B. Kumar, R. Kapadia, Q. Gao, M. Madsen, H. S. Kim, C.-H. Liu, Y.-L. Chueh, E. Plis, S. Krishna, H. A. Bechtel, J. Guo, and A. Javey, Quantum Confinement Effects in Nanoscale-Thickness InAs Membranes, Nano Lett. 2011;11:5008.

[87] S.-H. Kim, M. Yokoyama, N. Taoka, R. Nakane, T. Yasuda, M. Ichikawa, N. Fukuhara, M. Hata, M. Takenaka, and S. Takagi, Enhancement Technologies and Physical Understanding of Electron Mobility in III-V n-MOSFETs with Strain and MOS Interface Buffer Engineering, In: IEDM Tech. Dig. 2011; p. 311-314.

[88] K. Takei, M. Madsen, H. Fang, R. Kapadia, S. Chuang, H.-S. Kim, C.-H. Liu, E. Plis, J. Nah, S. Krishna, Y.-L. Chueh, J. Guo, and A. Javey, Nanoscale InGaSb heterostructure membranes on Si substrates for high hole mobility transistors, Nano Lett. $2012 ; 12: 2060$. 
[89] S.-H. Kim, M. Yokoyama, N. Taoka, R. Iida, S.-H. Lee, R. Nakane, Y. Urabe, N. Miyata, T. Yasuda, H. Yamada, N. Fukuhara, M. Hata, M. Takenaka, and S. Takagi, Electron Mobility Enhancement of Extremely Thin Body In0.7Ga0.3As-on-Insulator Metal-Oxide-Semiconductor Field-Effect Transistors on $\mathrm{Si}$ Substrates by Metal-Oxide-Semiconductor Interface Buffer Layers, Appl. Phys. Express 2012;5:014201.

[90] S.-H. Kim, M. Yokoyama, N. Taoka, R. Nakane, T. Yasuda, O. Ichikawa, N. Fukuhara, M. Hata, M. Takenaka, and S. Takagi, Sub-60 nm Deeply-Scaled Channel Length Extremely-thin Body $\mathrm{In}_{\mathrm{x}} \mathrm{Ga} \mathrm{1}_{-\mathrm{x}} \mathrm{As}-\mathrm{On}$-Insulator MOSFETs on Si with Ni-InGaAs Metal S/D and MOS Interface Buffer Engineering, In: Symp. VLSI Tech. Dig. 2012; p. 177-178.

[91] M. Yokoyama, S.-H. Kim, R. Zhang, N. Taoka, Y. Urabe, T. Maeda, H. Takagi, T. Yasuda, H. Yamada, O. Ichikawa, N. Fukuhara, M. Hata, M. Sugiyama, Y. Nakano, M. Takenaka, and S. Takagi, III-V/Ge High Mobility Channel Integration of InGaAs n-Channel and Ge p-Channel Metal-Oxide-Semiconductor Field-Effect Transistors with Self-Aligned Ni-Based Metal Source/Drain Using Direct Wafer Bonding, Appl. Phys. Express. 2012;5:076501.

[92] L. Czornomaz, N. Daix, D. Caimi, M. Sousa, R. Erni, M. D. Rossell, M. El-Kazzi, C. Rossel, C. Marchiori, E. Uccelli, M. Richter, H. Siegwart, and J. Fompeyrine, An Integration Path for Gate-first UTB III-V-on-insulator MOSFETs with Silicon, using Direct Wafer Bonding and Donor Wafer Recycling, IEDM Tech. Dig., 2012, p. 517.

[93] T. Irisawa, M. Oda, Y. Kamimuta, Y. Moriyama, K. Ikeda, E. Mieda, W. Jevasuwan, T. Maeda, O. Ichikawa, T. Osada, M. Hata, and T. Tezuka, Demonstration of InGaAs/Ge Dual Channel CMOS Inverters with High Electron and Hole Mobility Using Staked 3D 
Integration, In: Symp. VLSI Tech. Dig., 2013; p. T56-T57.

[94] S.-H. Kim, M. Yokoyama, N. Taoka, R. Nakane, T. Yasuda, O. Ichikawa, N. Fukuhara, M. Hata, M. Takenaka, and S. Takagi, Experimental Study on Electron Mobility in $\operatorname{In}_{x} \mathrm{Ga}_{1-\mathrm{x}} \mathrm{As}$-on-Insulator Metal-Oxide-Semiconductor Field-Effect Transistors With In Content Modulation and MOS Interface Buffer Engineering, IEEE Trans. on Nanotechnology 2013;12:621.

[95] M. Yokoyama, R. Iida, Y. Ikku, S.-H. Lee, S.-H. Kim, N. Taoka, Y. Urabe, T. Yasuda, H. Takagi, H. Yamada, N. Fukuhara, M. Hata, M. Sugiyama, Y. Nakano, M. Takenaka, and S. Takagi, Formation of III-V-on-insulator structures on Si by direct wafer bonding, Semicond. Sci. Technol. 2013;28:094009.

[96] S.-H. Kim, M. Yokoyama, R. Nakane, O. Ichikawa, T. Osada, M. Hata, M. Takenaka, and S. Takagi, Biaxially strained extremely-thin body $\mathrm{In}_{0.53} \mathrm{Ga} 0.47 \mathrm{As}-$ on-insulator metal-oxide-semiconductor field-effect transistors on $\mathrm{Si}$ substrate and physical understanding on their electron mobility, J. Appl. Phys. $2013 ; 114: 164512$.

[97] L. Czornomaz, N. Daix, K. Cheng, D. Caimi, C. Rossel, K. Lister, M Sousa, and J. Fompeyrine, Co-Integration of InGaAs n- and SiGe p-MOSFETs into Digital CMOS Circuits Using Hybrid Dual-Channel ETXOI Substrates, In: IEDM Tech. Dig. 2013; p. $52-55$.

[98] M. Yokoyama, H. Yokoyama, M. Takenaka, and S. Takagi, Ultrathin body GaSb-on-insulator p-channel metal-oxide-semiconductor field-effect transistors on $\mathrm{Si}$ fabricated by direct wafer bonding, Appl. Phys. Lett. 2015;106:073503.

[99] S.-H. Kim, Y. Ikku, M. Yokoyama, R. Nakane, J. Li, Y. C. Kao, M. Takenaka and S. Takagi, High Performance InGaAs-On-Insulator MOSFETs on Si by Novel Direct 
Wafer Bonding Technology applicable to Large Wafer Size Si, In: Symp. VLSI Tech. Dig. 2014; p. 38-39.

[100] K. Nishi, M. Yokoyama, H. Yokoyama, T. Hoshi, H. Sugiyama, M. Takenaka, and

S. Takagi, High hole mobility front-gate InAs/InGaSb-OI single structure CMOS on Si, In: Symp. VLSI Tech. Dig. 2015; p. T174-T175

[101] S.-H. Kim, Y. Ikku, M. Yokoyama, R. Nakane, J. Li, Y. C. Kao, M. Takenaka and S. Takagi, Direct wafer bonding technology for large-scale InGaAs-on-insulator transistors, Appl. Phys. Lett. 2014;105:043504.

[102] N. Daix, E. Uccelli, L. Czornomaz, D. Caimi, C. Rossel, M. Sousa, H. Siegwart, C. Marchiori, J. M. Hartmann, K.-T. Shiu, C.-W. Cheng, M. Krishnan, M. Lofaro, M. Kobayashi, D. Sadana and J. Fompeyrine, APL Mater. 2014;2:086104.

[103] V. Deshpande, V. Djara, E. O’Connor, P. Hashemi, K. Balakrishnan, M. Sousa, D. Caimi, A. Olziersky, L. Czornomaz and J. Fompeyrine, Advanced 3D Monolithic Hybrid CMOS with Sub-50 nm Gate Inverters Featuring Replacement Metal Gate (RMG)-InGaAs nFETs on SiGe-OI Fin pFETs; In: IEDM Tech. Dig. 2015; p. 209-212. [104] C. Wilmsen, Physics and Chemistry of III-V Compound Semiconductor Interfaces, Plenum Press, New York, 1985.

[105] S. Oktyabrsky and P. Ye, Fundamentals of III-V Semiconductor MOSFETs, Springer-Verlag, New York, 2010.

[106] M. M. Frank, G. D. Wilk, D. Starodub, T. Gustafsson, E. Garfunkel, Y. J. Chabal, J. Grazul, and D. A. Muller, $\mathrm{HfO}_{2}$ and $\mathrm{Al}_{2} \mathrm{O}_{3}$ gate dielectrics on $\mathrm{GaAs}$ grown by atomic layer deposition, Appl. Phys. Lett. 2005;86:152904.

[107] M. L. Huang, Y. C. Chang, C. H. Chang, Y. J. Lee, P. Chang, J. Kwo, T. B. Wu, and M. Hong, Surface passivation of III-V compound semiconductors using 
atomic-layer-deposition-grown $\mathrm{Al}_{2} \mathrm{O}_{3}$, Appl. Phys. Lett. 2005;87:252104.

[108] C.-H. Chang, Y.-K. Chiou, Y.-C. Chang, K.-Y. Lee, T.-D. Lin, T.-B. Wu, M. Hong, and J. Kwo, Interfacial self-cleaning in atomic layer deposition of $\mathrm{HfO}_{2}$ gate dielectric on In0.15Ga0.85As, Appl. Phys. Lett. 2006;89:242911.

[109] M. Milojevic, F. S. Aguirre-Tostado, C. L. Hinkle, H. C. Kim, E. M. Vogel, J. Kim, and R. M. Wallace, Half-Cycle Atomic Layer Deposition Reaction Studies of $\mathrm{Al}_{2} \mathrm{O}_{3}$ on $\mathrm{In}_{0.2} \mathrm{Ga}_{0.8} \mathrm{As}$ (100) Surfaces, Appl. Phys. Lett. 2008;93:202902.

[110] Y. Xuan, Y. Q. Wu, and P. D. Ye, High-performance inversion-type enhancement-mode InGaAs MOSFET with maximum drain current exceeding $1 \mathrm{~A} / \mathrm{mm}$, IEEE Electron Device Lett. 2008;29:294-296.

[111] M. Egard, L. Ohlsson, B. M. Borg, F. Lenrick, R. Wallenberg, L.-E. Wernersson, and E. Lind, High Transconductance Self-Aligned Gate-Last Surface Channel In0.53Ga0.47As MOSFET, In: IEDM Tech. Dig. 2011; p. 303-306.

[112] Y. Yonai, T. Kanazawa, S. Ikeda, and Y. Miyamoto, High Drain Current $(>2 \mathrm{~A} / \mathrm{mm})$ InGaAs Channel MOSFET at $\mathrm{V}_{\mathrm{D}}=0.5 \mathrm{~V}$ with Shrinkage of Channel Length by InP Anisotropic Etching, In: IEDM Tech. Dig. 2011; p. 307-310.

[113] R. Suzuki, N. Taoka, S. Lee, S. H. Kim, T. Hoshii, M. Yokoyama, T. Yasuda, W. Jevasuwan, T. Maeda, O. Ichikawa, N. Fukuhara, M. Hata, M. Takenaka, and S. Takagi, 1-nm-capacitance-equivalent-thickness $\mathrm{HfO}_{2} / \mathrm{Al}_{2} \mathrm{O}_{3} / \mathrm{InGaAs}$ metal-oxide-semiconductor structure with low interface trap density and low gate leakage current density, Appl. Phys. Lett. 2012;100:132906.

[114] R. Suzuki, N. Taoka, M. Yokoyama, S.-H. Kim, T. Hoshii, T. Maeda, T. Yasuda, O. Ichikawa, N. Fukuhara, M. Hata, M. Takenaka, and S. Takagi, Impact of atomic layer deposition temperature on $\mathrm{HfO}_{2} / \mathrm{InGaAs}$ metal-oxide-semiconductor interface properties, 
J. Appl. Phys. 2012;112: 084103.

[115] A. O'Mahony, S. Monaghan, G. Provenzano, I. M. Povey, M. G. Nolan, E. O'Connor, K. Cherkaoui, S. B. Newcomb, F. Crupi, P. K. Hurley, and M. E. Pemble, Structural and electrical analysis of the atomic layer deposition of $\mathrm{HfO}_{2} / \mathrm{n}-\mathrm{In}_{0.53} \mathrm{Ga} 0.47 \mathrm{As}$ capacitors with and without an $\mathrm{Al}_{2} \mathrm{O}_{3}$ interface control layer, Appl. Phys. Lett. 2010;97:052904.

[116] S. Monaghan, A. O'Mahony, K. Cherlaoui, E. O'Connor, I. M. Povey, M. G. Nolan, D. O'Connell, M. E. Pemble, and P. K. Hurley, Electrical analysis of three-stage passivated $\mathrm{In}_{0.53} \mathrm{Ga}_{0.47}$ As capacitors with varying $\mathrm{HfO}_{2}$ thicknesses and incorporating an Al2O3Al2O3 interface control layer, J. Vac. Sci. Technol. B 2011;29:01A807.

[117] D. H. Zadeh, H. Oomine, K. Kakushima, Y. Kataoka, A. Nishiyama, N. Sugii, H. Wakabayashi, K. Tsutsui, K. Natori, and H. Iwai, Low Dit high-k/In ${ }_{0.53} \mathrm{Ga} 0.47 \mathrm{As}$ Gate Stack, with CET Down to $0.73 \mathrm{~nm}$ and Thermally Stable Silicide Contact by Suppression of Interfacial Reaction, In: IEDM Tech. Dig. 2011; p. 36-39.

[118] C.-Y. Chang, O. Ichikawa, T. Osada, M. Hata, H. Yamada, M. Takenaka, and S. Takagi, Impact of $\mathrm{La}_{2} \mathrm{O}_{3}$ interfacial layers on InGaAs metal-oxide-semiconductor interface properties in $\mathrm{Al}_{2} \mathrm{O}_{3} / \mathrm{La}_{2} \mathrm{O}_{3} / \mathrm{InGaAs}$ gate stacks deposited by atomic-layer-deposition, J. Appl. Phys. 2015;118:085309.

[119] J. Robertson and L. Lin, "Bonding principles of passivation mechanism at III-V-oxide interfaces”, Appl. Phys. Lett. 2011;99: 222906.

[120] M. Yokoyama, K. Nishi, S.-H. Kim, H. Yokoyama, M. Takenaka, and S. Takagi, Self-aligned Ni-GaSb source/drain junctions for $\mathrm{GaSb}$ p-channel metal-oxide semiconductor field-effect transistors, Appl. Phys. Lett. 2014;104:093509.

[121] A. Nainani, S. Raghunathan, D. Witte, M. Kobayashi, T. Irisawa, T. 
Krishnamohan, and K. Saraswat, Engineering of Strained III-V Heterostructures for High Hole Mobility, In: IEDM Tech. Dig. 2009; p. 857-860.

[122] A. Nainani, T. Irisawa, Z. Yuan, Y. Sun, T. Krishnamohan, M. Reason, B. R. Bennett, J. B. Boos, M. G. Ancona, Y. Nishi, and K. C. Saraswat, Development of high-k dielectric for Antimonides and a sub $350^{\circ} \mathrm{C}$ III-V pMOSFET outperforming Germanium, In: IEDM Tech. Dig. 2010; p. 138-141.

[123] A. Nainani, T. Irisawa, Z. Yuan, B. R. Bennett, J. B. Boos, Y. Nishi, and K. C. Saraswat, Optimization of the $\mathrm{Al}_{2} \mathrm{O}_{3} / \mathrm{GaSb}$ Interface and a High-Mobility $\mathrm{GaSb}$ pMOSFET, IEEE Trans. Electron Devices 2011;8:3407.

[124] M. Xu, R. Wang, and P. D. Ye, GaSb Inversion-Mode PMOSFETs With Atomic-Layer-Deposited as Gate Dielectric, IEEE Electron Device Lett. 2011;32:883. [125] Z. Yuan, A. Kumar, C.-Y. Chen, A. Nainani, B. R. Bennett, J. B. Boos, and K. C. Saraswat, Antimonide-based heterostructure p-channel MOSFETs with Ni-alloy source/drain, IEEE Electron Device Lett. 2013;34:1367.

[126] R. L. Chu, T. H. Chiang, W. J. Hsueh, K. H. Chen, K. Y. Lin, G. J. Brown, J. I. Chyi, J. Kwo, and M. Hong, Passivation of GaSb using molecular beam epitaxy $\mathrm{Y}_{2} \mathrm{O}_{3}$ to achieve low interfacial trap density and high-performance self-aligned inversion-channel p-metal-oxide-semiconductor field-effect-transistors, Appl. Phys. Lett. 2014;105:182106.

[127] K. Nishi, M. Yokoyama, H. Yokoyama, T. Hoshi, H. Sugiyama, M. Takenaka and S. Takagi, Operation of the GaSb p-channel metal-oxide-semiconductor field-effect transistors fabricated on (111)A surfaces, Appl. Phys. Lett. 2014;105:233503.

[128] A. Nainani, Z. Yuan, T. Krishnamohan, B. R. Bennett, J. B. Boos, M. Reason, M. G. Ancona, Y. Nishi, and K. C. Saraswat, $\operatorname{In}_{\mathrm{x}} \mathrm{Ga}_{1-\mathrm{x}} \mathrm{Sb}$ channel p-metal-oxide- 
semiconductor field effect transistors: Effect of strain and heterostructure design, J. Appl. Phys. 2011;110:014503.

[129] Z. Yuan, A. Nainani, B. R. Bennett, J. B. Boos, M. G. Ancona, and K. C. Saraswat, Amelioration of interface state response using band engineering in III-V quantum well metal-oxide-semiconductor field-effect transistors, Appl. Phys. Lett. 2012;100:143503. [130] S. Datta, T. Ashley, J. Brask, L. Buckle, M. Doczy, M. Emeny, D. Hayes, K. Hilton, R. Jefferies, T. Martin, T. J. Phillips, D. Wallis, P. Wilding, and R. Chau, 85nm Gate Length Enhancement and Depletion mode InSb Quantum Well Transistors for Ultra High Speed and Very Low Power Digital Logic Applications, In: IEDM Tech. Dig. 2005; p. 763-766.

[131] M. Radosavljevic, T. Ashley, A. Andreev, S. D. Coomber, G. Dewey, M. T. Emeny, M. Fearn, D. G. Hayes, K. P. Hilton, M. K. Hudait, R. Jefferies, T. Martin, R. Pillarisetty, W. Rachmady, T. Rakshit, S. J. Smith, M. J. Uren, D. J. Wallis, P. J. Wilding, and R. Chau, High-Performance 40nm Gate Length InSb P-Channel Compressively Strained Quantum Well Field Effect Transistors for Low-Power $\left(\mathrm{V}_{\mathrm{CC}}=0.5 \mathrm{~V}\right)$ Logic Applications, In: IEDM Tech. Dig. 2008; p. 727-730.

[132] T. Ashley, M. T. Emeny, D. G. Hayes, K. P. Hilton, R. Jefferies, J. O. Maclean, S. J. Smith, A. W-H. Tang, D. J. Wallis, and P. J. Webber, High-Performance InSb Based Quantum Well Field Effect Transistors for Low-Power Dissipation Applications, In: IEDM Tech. Dig. 2009; p. 849-852.

[133] M. Yokoyama, H. Yokoyama, M. Takenaka, and S. Takagi, Impact of process temperature on $\mathrm{GaSb}$ metal-oxide-semiconductor interface properties fabricated by ex-situ process, Appl. Phys. Lett. 2014; 104:262901.

[134] A. Greene, S. Madisetti, P. Nagaiah, M. Yakimov, V. Tokranov, R. Moore, and S. 
Oktyabrsky, Improvement of the $\mathrm{GaSb} / \mathrm{Al}_{2} \mathrm{O}_{3}$ interface using a thin InAs surface layer, Solid State Electron. 2012;78:56.

[135] M. Yokoyama, H. Yokoyama, M. Takenaka and S. Takagi, Impact of interfacial InAs layers on $\mathrm{Al}_{2} \mathrm{O}_{3} / \mathrm{GaSb}$ metal-oxide-semiconductor interface properties, Appl. Phys. Lett. 2015; 106:122902.

[136] K. Nishi, M. Yokoyama, H. Yokoyama, T. Hoshi, H. Sugiyama, M. Takenaka and S. Takagi, Effects of buffered HF cleaning on metal-oxide-semiconductor interface properties of A12O3/InAs/GaSb structures, Appl. Phys. Express, 2015;8:061203.

[137] K. Uchida and S. Takagi, Carrier scattering induced by thickness fluctuation of silicon-on-insulator film in ultrathin-body metal-oxide-semiconductor field-effect transistors, Appl. Phys. Lett. 2003;82:2916.

[138] K. Uchida, J. Koga, and S. Takagi, Experimental study on electron mobility in ultrathin-body silicon-on-insulator metal-oxide-semiconductor field-effect transistors, J. Appl. Phys. 2007;102:074510.

[139] S.-H. Kim, M. Yokoyama, O. Ichikawa, T. Osada, M. Hata, M. Takenaka, and S. Takagi, Experimental study on vertical scaling of InAs-on-insulator metal-oxide-semiconductor field-effect transistors, Appl. Phys. Lett. 2014;104:263507. [140] S. H. Kim, M. Yokoyama, R. Nakane, O. Ichikawa, T. Osada, M. Hata, M. Takenaka, and S. Takagi, High Performance Extremely-Thin Body InAs-On-Insulator MOSFETs on $\mathrm{Si}$ with Ni-InGaAs Metal $\mathrm{S} / \mathrm{D}$ by Contact Resistance Reduction Technology, In: Symp. VLSI Tech. Dig. 2013; T52-T53.

[141] B. Duriez, G. Vellianitis, M. J. H. van Dal, G. Doornbos, R. Oxland, K. K. Bhuwalka, M. Holland, Y. S. Chang, C. H. Hsieh, K. M. Yin, Y. C. See, M. Passlack, C. H. Diaz, Scaled p-channel Ge FinFET with optimized gate stack and record 
performance integrated on 300mm Si wafers, In: IEDM Tech. Dig. 2013; p. 522-525

[142] L. Witters, J. Mitard, R. Loo, G. Eneman, H. Mertens, D.P. Brunco1, S.H. Lee, N. Waldron, A. Hikavyy, P. Favia, A.P. Milenin, Y. Shimura, C. Vrancken, H. Bender, N. Horiguchi, K. Barla, A. Thean, N. Collaert, Strained Germanium Quantum Well pMOS FinFETs Fabricated on in situ Phosphorus-Doped SiGe Strain Relaxed Buffer Layers Using a Replacement Fin Process, In: IEDM Tech. Dig. 2013; p. 534-537

[143] P. Hashemi, K. Balakrishnan, A. Majumdar, A. Khakifirooz, W. Kim, A. Baraskar, L. A. Yang, K. Chan, S. U. Engelmann, J. A. Ott, D. A. Antoniadis, E. Leobandung, D.-G. Park, Strained Si1-xGe $\mathrm{Ge}_{\mathrm{x}}$-on-Insulator PMOS FinFETs with Excellent Sub-Threshold Leakage, Extremely-High Short-Channel Performance and Source Injection Velocity for 10nm Node and Beyond, In: Symp. VLSI Tech. Dig. 2014; p. $17-18$.

[144] J. Mitard, L. Witters, R. Loo, S.H. Lee1, J.W. Sun, J. Franco, L.-Å. Ragnarsson, A. Brand, X. Lu, N. Yoshida, G. Eneman, D. P. Brunco, M. Vorderwestner, P. Storck, A. P. Milenin, A. Hikavyy, N. Waldron, P. Favia, D. Vanhaeren, A. Vanderheyden, R. Olivier, H. Mertens, H. Arimura, S. Sonja, C. Vrancken, H. Bender, P. Eyben, K. Barla, S-G Lee, N. Horiguchi, N. Collaert, A. V-Y. Thean, 15nm-WFIN High-Performance Low-Defectivity Strained-Germanium pFinFETs With Low Temperature STI-Last Process, In: Symp. VLSI Tech. Dig. 2014; p. 110-111.

[145] P. Hashemi, K. Balakrishnan, S. U. Engelmann, J. A. Ott, A. Khakifirooz, A. Baraskar, M. Hopstaken, J. S. Newbury, K. K. Chan, E. Leobandung, R. T. Mo and D.-G. Park, First Demonstration of High-Ge-Content Strained-Si1-x $\mathrm{Ge}_{\mathrm{x}}(\mathrm{x}=0.5)$ on Insulator PMOS FinFETs with High Hole Mobility and Aggressively Scaled Fin Dimensions and Gate Lengths for High-Performance Applications, In: IEDM Tech. Dig. 2014; p. 
$402-405$.

[146] A. Agrawal, M. Barth, G. B. Rayner Jr., Arun V. T., C. Eichfeld, G. Lavallee, S-Y. Yu, X. Sang, S. Brookes, Y. Zheng, Y-J. Lee, Y-R. Lin, C-H. Wu, C-H. Ko, J. LeBeau, R. Engel-Herbert, S. E. Mohney, Y-C. Yeo and S. Datta, Enhancement Mode Strained (1.3\%) Germanium Quantum Well FinFET (WFin=20nm) with High Mobility $\left(\mu_{\mathrm{Hole}}=700 \mathrm{~cm}^{2} / \mathrm{Vs}\right)$, Low EOT $(\sim 0.7 \mathrm{~nm})$ on Bulk Silicon Substrate, In: IEDM Tech. Dig. 2014; p. 414-417.

[147] P. Hashemi, T. Ando, K. Balakrishnan, J. Bruley, S. Engelmann, J. A. Ott, V. Narayanan, D.-G. Park, R.T. Mo, E. Leobandung, High-Mobility High-Ge-Content $\mathrm{Si}_{1-\mathrm{x}} \mathrm{Ge}_{\mathrm{x}}$-OI PMOS FinFETs with Fins Formed Using 3D Germanium Condensation with Ge Fraction up to $\mathrm{x} \sim 0.7$, Scaled EOT $\sim 8.5 \AA$ and $\sim 10 \mathrm{~nm}$ Fin Width, In: Symp. VLSI Tech. Dig. 2015; p. T16-T17.

[148] L. Witters, J. Mitard, R. Loo, S. Demuynck, S.A. Chew, T. Schram, Z. Tao, A. Hikavyy, J.W. Sun, A. P. Milenin, H. Mertens, C. Vrancken, P. Favia, M. Schaekers, H. Bender, N. Horiguchi, R. Langer, K. Barla, D. Mocuta, N. Collaert, A. V-Y. Thean, Strained germanium quantum well p-FinFETs fabricated on $45 \mathrm{~nm}$ Fin pitch using replacement channel, replacement metal gate and germanide-free local interconnect, In: Symp. VLSI Tech. Dig. 2015; p. T56-T57.

[149] H. Mertens, R. Ritzenthaler, H. Arimura, J. Franco, F. Sebaai, A. Hikavyy, B. J. Pawlak, V. Machkaoutsan, K. Devriendt, D. Tsvetanova, A. P. Milenin, L. Witters, A. Dangol, E. Vancoille, H. Bender, M. Badaroglu, F. Holsteyns, K. Barla, D. Mocuta, N. Horiguchi, A. V-Y Thean, Si-cap-free SiGe p-Channel FinFETs and Gate-All-Around Transistors in a Replacement Metal Gate Process: Interface Trap Density Reduction and Performance Improvement by High-Pressure Deuterium Anneal, In: Symp. VLSI Tech. 
Dig. 2015; p. T142-T143.

[150] K. Ikeda, Y. Kamimuta, Y. Moriyama, M. Ono, K. Usuda, M. Oda, T. Irisawa, K. Furuse, and T. Tezuka, Enhancement of Hole Mobility and Cut-off Characteristics of Strained Ge Nanowire pMOSFETs by using Plasma Oxidized $\mathrm{GeO}_{\mathrm{x}}$ Inter-Layer for Gate Stack, In: Symp. VLSI Tech. Dig. 2013; p. T30-T31.

[151] Y.-J. Lee, F.-J. Hou, S.-S. Chuang, F.-K. Hsueh, K.-H. Kao, P.-J. Sung, W.-Y. Yuan, J.-Y. Yao, Y.-C. Lu, K.-L. Lin, C.-T. Wu, H.-C. Chen, B.-Y. Chen, G.-W. Huang, H. J. H. Chen, J.-Y. Li, Y. Li, S. Samukawa, T.-S. Chao, T.-Y. Tseng, W.-F. Wu, T.-H. Hou and W.-K. Yeh, Diamond-shaped Ge and Ge0.9Si0.1 Gate-All-Around Nanowire FETs with Four $\{111\}$ Facets by Dry Etch Technology, In: IEDM Tech. Dig. 2015; p. 382-385.

[152] H. Wu, W. Wu, M. Si and P. D. Ye, First Demonstration of Ge Nanowire CMOS Circuits: Lowest SS of $64 \mathrm{mV} / \mathrm{dec}$, Highest $\mathrm{g}_{\max }$ of $1057 \mu \mathrm{S} / \mu \mathrm{m}$ in Ge nFETs and Highest Maximum Voltage Gain of $54 \mathrm{~V} / \mathrm{V}$ in Ge CMOS inverters, In: IEDM Tech. Dig. 2015; p. 16-19.

[153] D. Kuzum, A. J. Pethe, T. Krishnamohan, Y. Oshima, Y. Sun, J. P. McVittie, P. A. Pianetta, P. C. McIntyre and K. C. Saraswat, Interface-Engineered Ge (100) and (111), N- and P-FETs with High Mobility, In: IEDM Tech. Dig. 2007; p. 723-726.

[154] D. Kuzum, A. J. Pethe, T. Krishnamohan, and K. C. Saraswat, Ge (100) and (111) $\mathrm{N}$ - and P-FETs with high mobility and low-T mobility characterization, IEEE Trans. Electron Device. 2009;56:648-655.

[155] C. H. Lee, T. Nishimura, N. Saido, K. Nagashio, K. Kita and A. Toriumi, Record-high Electron Mobility in. Ge n-MOSFETs Exceeding Si Universality, In: IEDM Tech. Dig. 2009; p. 457-460. 
[156] K. Morii, T. Iwasaki, R. Nakane, M. Takenaka and S. Takagi, "High Performance $\mathrm{GeO}_{2} / \mathrm{Ge}$ nMOSFETs with Source/Drain Junctions Formed by Gas Phase Doping”, In: IEDM Tech. Dig. 2009; p. 681-684.

[157] Y. Kamata, K. Ikeda, Y. Kamimuta, and T. Tezuka, High-k/Ge p- \& n- MISFETs with strontium germanide interlayer for EOT scalable CMIS application, In: Symp. VLSI Tech. Dig. 2010; p. 211-212.

[158] K. Morii, T. Iwasaki, R. Nakane, M. Takenaka and S. Takagi, High Performance $\mathrm{GeO}_{2} / \mathrm{Ge}$ nMOSFETs with Source/Drain Junctions Formed by Gas Phase Doping, IEEE Electron Device Lett. 2010;31:1092-1094.

[159] C. H. Lee, T. Nishimura, T. Tabata, S. K. Wang, K. Nagashio, K. Kita, and A. Toriumi, Ge MOSFETs performance: Impact of Ge interface passivation, In: IEDM Tech. Dig. 2010; p. 416-419.

[160] D. Kuzum, T. Krishnamohan, A. Nainani, Y. Sun, P. A. Pianetta, H. S-.P. Wong, and K. C. Saraswat, High-Mobility Ge N-MOSFETs and Mobility Degradation Mechanisms, IEEE Trans. Electron Devices 2011;58:59-66.

[161] C. H. Lee, T. Nishimura, K. Nagashio, K. Kita, and A. Toriumi, High electron-mobility $\mathrm{Ge} / \mathrm{GeO}_{2}$ n-MOSFETs with tow-step oxidation, IEEE Trans. Electron Devices 2011;58:1295-1301.

[162] R. Zhang, N. Taoka, P. C. Huang, M. Takenaka, and S. Takagi, 1-nmthick EOT high mobility Ge n- and p-MOSFETs with ultrathin $\mathrm{GeO}_{x} / \mathrm{Ge}$ MOS interfaces fabricated by plasma post oxidation, In: IEDM Tech. Dig. 2011; p. 642-645.

[163] R. Zhang, N. Taoka, P. C. Huang, M. Takenaka, and S. Takagi, High mobility Ge pMOSFET with 1-nm EOT $\mathrm{Al}_{2} \mathrm{O}_{3} / \mathrm{GeO}_{x} / \mathrm{Ge}$ gate stack fabricated by plasma post oxidation, IEEE Trans. Electron. Devices 2012;59:335-341. 
[164] R. Zhang, P.-C. Huang, J.-C. Lin, M. Takenaka, and S. Takagi, Physical mechanism determining Ge p- and n-MOSFETs mobility in high Ns region and mobility improvement by atomically flat $\mathrm{GeO}_{x} / \mathrm{Ge}$ interfaces, In: IEDM Tech. Dig. 2012; p. $371-374$.

[165] S.-H. Hsu, H.-C. Chang, C.-L. Chu, Y.-T. Chen, W.-H. Tu, F. J. Hou, C. H. Lo, P.-J. Sung, B.-Y. Chen, G.-W. Huang, G.-L. Luo, C. W. Liu, C. Hu and F.-L. Yang, Triangular-channel Ge NFETs on Si with (111) Sidewall-Enhanced Ion and Nearly Defect-free Channels, In: IEDM Tech. Dig. 2012; p. 525-528.

[166] R. Zhang, P.-C. Huang, J.-C. Lin, N. Taoka, M. Takenaka and S. Takagi, High Mobility Ge p- and n-MOSFETs with $0.7 \mathrm{~nm}$ Ultrathin EOT using $\mathrm{HfO}_{2} / \mathrm{Al}_{2} \mathrm{O}_{3} / \mathrm{GeO}_{\mathrm{x}} / \mathrm{Ge}$ Gate Stacks Fabricated by Plasma Post Oxidation, IEEE Trans. Electron Devices 2013;60:927-934.

[167] R. Zhang, J-C. Lin, X. Yu, M. Takenaka and S. Takagi, Examination of Physical Origins Limiting Effective Mobility of Ge MOSFETs and the Improvement by Atomic Deuterium Annealing, In: Symp. VLSI Tech. Dig. 2013; p. T26-T27.

[168] C. H. Lee, C. Lu, T. Tabata, T. Nishimura, K. Nagashio, and A. Toriumi, Enhancement of high- $\mathrm{N}_{\mathrm{s}}$ electron mobility in sub-nm EOT Ge n-MOSFETs, In: Symp. VLSI Tech. Dig. 2013, p. T28-T29.

[169] C. H. Lee, T. Nishimura, T. Tabata, C. Lu, W. F. Zhang, K. Nagashio, and A. Toriumi, Reconsideration of Electron Mobility in Ge n-MOSFETs from Ge Substrate Side -Atomically flat surface formation, layer-by-layer oxidation, and dissolved oxygen extraction-, In: IEDM Tech. Dig. 2013; p. 40-43.

[170] R. Zhang, J.-C. Lin, X. Yu, M. Takenaka, and S. Takagi, Impact of plasma postoxidation temperature on the electrical properties of $\mathrm{Al}_{2} \mathrm{O}_{3} / \mathrm{GeO}_{\mathrm{x}} / \mathrm{Ge}$ pMOSFETs 
and nMOSFETs, IEEE Trans. Electron Devices 2014;61:416-422.

[171] C. H. Lee, C. Lu, T. Nishimura, K. Nagashio, and A. Toriumi, Thermally Robust CMOS-aware Ge MOSFETs with High Mobility at High-carrier Densities on a Single Orientation Ge Substrate, In: Symp. VLSI Tech. Dig. 2014; p. 116-117.

[172] H. Wu, M. Si, L. Dong, J. Zhang and P. D. Ye, Ge CMOS: Breakthroughs of nFETs $\left(\mathrm{I}_{\max }=714 \mathrm{~mA} / \mathrm{mm}, \mathrm{g}_{\max }=590 \mathrm{mS} / \mathrm{mm}\right)$ by recessed channel and $\mathrm{S} / \mathrm{D}$, In: Symp. VLSI Tech. Dig. 2014; p. 76-77.

[173] R. Zhang, X. Yu, M. Takenaka and S. Takagi, Physical Origins of High Normal Field Mobility Degradation in Ge p- and n-MOSFETs with $\mathrm{GeO}_{\mathrm{x}} / \mathrm{Ge}$ MOS Interfaces Fabricated by Plasma Post Oxidation, IEEE Trans. Electron Devices 2014;61: $2316-2323$.

[174] R. Zhang, X. Yu, M. Takenaka and S. Takagi, Impact of Channel Orientation on Electrical Properties of Ge p- and n-MOSFETs with 1-nm EOT $\mathrm{Al}_{2} \mathrm{O}_{3} / \mathrm{GeO}_{x} / \mathrm{Ge}$ Gate Stacks Fabricated by Plasma Post Oxidation, IEEE Trans. Electron Devices 2014;61: $3668-3675$.

[175] H. Wu, N. Conrad, W. Luo, and P. D. Ye, First Experimental Demonstration of Ge CMOS Circuits, In: IEDM Tech. Dig. 2014; p. 227-230.

[176] H. Wu, W. Luo, M. Si, J. Zhang, H. Zhou and P. D. Ye, Deep Sub-100 nm Ge CMOS Devices on Si with the Recessed S/D and Channel, In: IEDM Tech. Dig. 2014; p. 426-429.

[177] X. Gong, Q. Zhou, M. H. S. Owen, X. Xu, D. Lei, S.-H. Chen, G. Tsai, C.-C. Cheng, Y.-R. Lin, C.-H. Wu, C.-H. Ko, and Y.-C. Yeo, InAlP-Capped (100) Ge nFETs with 1.06 nm EOT: Achieving Record High Peak Mobility and First Integration on 300 mm Si Substrate, In: IEDM Tech. Dig. 2014; p. 231-234. 
[178] M. J. H. van Dal, B. Duriez, G. Vellianitis, G. Doornbos, R. Oxland, M. Holland, A. Afzalian, Y.C. See, M. Passlack, C.H. Diaz1, Ge n-channel FinFET with optimized gate stack and contacts, In: IEDM Tech. Dig. 2014; p. 235-238.

[179] I-H. Wong, Y.-T. Chen, S.-H. Huang, W.-H. Tu, Y.-S. Chen, T.-C. Shieh, T.-Y. Lin, H.-S. Lan and C. W. Liu, In-situ Doped and Tensily Stained Ge Junctionless Gate-all-around nFETs on SOI Featuring $\mathrm{I}_{\mathrm{on}}=828 \mu \mathrm{A} / \mu \mathrm{m}, \mathrm{I}_{\mathrm{on}} / \mathrm{I}_{\text {off }} \sim 1 \times 10^{5}, \mathrm{DIBL}=$ 16-54 mV/V, and 1.4X External Strain Enhancement, In: IEDM Tech. Dig. 2014; p. $239-242$

[180] J. Mitard, L. Witters, H. Arimura, Y. Sasaki, A.P. Milenin, R. Loo, A. Hikavyy, G. Eneman, P. Lagrain, H. Mertens, S. Sioncke, C. Vrancken, H. Bender, K. Barla, N. Horiguchi, A. Mocuta, N. Collaert, A. V-Y. Thean, First Demonstration of 15nm-WFIN Inversion-Mode Relaxed-Germanium n-FinFETs with Si-cap Free RMG and NiSiGe Source/Drain, In: IEDM Tech. Dig. 2014; p. 418-421.

[181] C. H. Lee, T. Nishimura, C. Lu, S. Kabuyanagi, and A. Toriumi, Dramatic Effects of Hydrogen-induced Out-diffusion of Oxygen from Ge Surface on Junction Leakage as well as Electron Mobility in n-channel Ge MOSFETs, In: IEDM Tech. Dig. 2014; p. $780-783$.

[182] H. Wu, W. Luo, H. Zhou, M. Si, J. Zhang and P. D. Ye, First Experimental Demonstration of Ge 3D FinFET CMOS Circuits, In: Symp. VLSI Tech. Dig. 2015; p. T58-T29.

[183] C. Lu, C. H. Lee, T. Nishimura and A. Toriumi, Design and Demonstration of Reliability-aware Ge Gate Stacks with 0.5 nm EOT, In: Symp. VLSI Tech. Dig. 2015; p. T18-T19.

[184] H. Arimura, S. Sioncke, D. Cott, J. Mitard, T. Conard, W. Vanherle, R. Loo, P. 
Favia, H. Bender, J. Meersschaut, L. Witters, H. Mertens, J. Franco, L.-Å. Ragnarsson, G. Pourtois, M. Heyns, A. Mocuta, N. Collaert and A.V.-Y. Thean, Ge nFET with high electron mobility and superior PBTI reliability enabled by monolayer-Si surface passivation and La-induced interface dipole formation, In: IEDM Tech. Dig. 2015; p. 588-591.

[185] R. Zhang, X. Yu, M. Takenaka and S. Takagi, Impact of Post Deposition Annealing Ambient on the Mobility of Ge nMOSFETs with 1-nm EOT $\mathrm{Al}_{2} \mathrm{O}_{3} / \mathrm{GeO}_{x} / \mathrm{Ge}$ Gate Stacks, IEEE Trans. Electron Devices 2016;63:558

[186] Y. Fukuda, T. Ueno, S. Hirono, and S. Hashimoto, Electrical characterization of germanium oxide/germanium interface prepared by electron-cyclotron-resonance plasma irradiation, Jpn. J. Appl. Phys. 2005;44, Part 1:6981.

[187] S. Takagi, T. Maeda, N. Taoka, M. Nishizawa, Y. Morita, K. Ikeda, Y. Yamashita, M. Nishikawa, H. Kumagai, R. Nakane, S. Sugahara, and N. Sugiyama, Gate dielectric formation and MIS interface characterization on Ge, Microelectron. Eng. 2007;84:2314. [188] A. Delabie, F. Bellenger, M. Houssa, T. Conard, S. V. Elshocht, M. Aymax, M. M. Heyns, and M. Meuris, Effective electrical passivation of Ge (100) for high-k gate dielectric layers using germanium oxide, Appl. Phys. Lett. 2007;91:082904.

[189] T. Takahashi, T. Nishimura, L. Chen, S. Sakata, K. Kita, and A. Toriumi, Proof of Ge-interfacing Concepts for Metal/High-k/Ge CMOS - Ge-intimate Material Selection and Interface Conscious Process Flow -, In: IEDM Tech. Dig. 2007; p. 697-700.

[190] K. Kita, S. Suzuki, H. Nomura, T. Takahashi, T. Nishimura, and A. Toriumi, Direct evidence of $\mathrm{GeO}$ volatilization from $\mathrm{GeO}_{2} / \mathrm{Ge}$ and impact of its suppression on $\mathrm{GeO}_{2} / \mathrm{Ge}$ metal-insulator-semiconductor characteristics, Jpn. J. Appl. Phys. $2008 ; 47: 2349$. 
[191] H. Matsubara, T. Sasada, M. Takenaka, and S. Takagi, Evidence of low interface trap density in $\mathrm{GeO}_{2} / \mathrm{Ge}$ metal-oxide-semiconductor structures fabricated by thermal oxidation, Appl. Phys. Lett. 2008;93: 032104.

[192] D. Kuzum, T. Krishnamohan, A. J. Pethe, A. K. Okyay, Y. Oshima, Y. Sun, J. P. McVittie, P. A. Pianetta, P. C. McIntyre, and K. C. Saraswat, Ge-interface engineering with ozone oxidation for low interface-state density, IEEE Electron Device Lett. 2008;29:328.

[193] R. Xie, T. H. Phung, W. He, Z. Sun, M. Yu, Z. Cheng, and C. Zhu, High Mobility High-k/Ge pMOSFETs with $1 \mathrm{~nm}$ EOT -New Concept on Interface Engineering and Interface Characterization, In: IEDM Tech. Dig. 2008; p. 393-396.

[194] Y. Nakakita, R. Nakane, T. Sasada, H. Matsubara, M. Takenaka, and S. Takagi, Interface-controlled Self-Align Source/Drain Ge pMOSFETs Using Thermally-Oxidized $\mathrm{GeO}_{2}$ Interfacial Layers, In: IEDM Tech. Dig. 2008; p. 877-880.

[195] T. Hosoi, K. Kutsuki, G. Okamoto, M. Saito, T. Shimura, and H. Watanabe, Origin of flatband voltage shift and unusual minority carrier generation in thermally grown $\mathrm{GeO}_{2} / \mathrm{Ge}$ metal-oxide-semiconductor devices, Appl. Phys. Lett. 2009;94:202112.

[196] C. H. Lee, T. Tabata, T. Nishimura, K. Nagashio, K. Kita, and A. Toriumi, $\mathrm{Ge} / \mathrm{GeO}_{2}$ Interface Control with High-Pressure Oxidation for Improving Electrical Characteristics, Appl. Phys. Exp. 2009;2:071404.

[197] T. Sasada, Y. Nakakita, M. Takenaka, and S. Takagi, Surface orientation dependence of interface properties of $\mathrm{GeO}_{2} / \mathrm{Ge}$ metal-oxide-semiconductor structures fabricated by thermal oxidation, J. Appl. Phys. 2009;106: 073716.

[198] D. Kuzum, T. Krishnamohan, A. Nainani, Y. Sun, P. A. Pianetta, H. S-.P. Wong, and K. C. Saraswat, Experimental Demonstration of High Mobility Ge NMOS, In: 
IEDM Tech. Dig. 2009; p. 453-456.

[199] T. Nishimura, C. H. Lee, S. K. Wang, T. Tabata, K. Kita, K. Nagashio, and A. Toriumi, Electron Mobility in High-k Ge-MISFETs Goes Up to Higher, In: Symp. VLSI Tech. Dig. 2010; p. 209-210.

[200] Y. Nakakita, R. Nakakne, T. Sasada, M. Takenaka, and S. Takagi, Interface-Controlled $\quad$ Self-Align $\quad$ Source/Drain $\quad \mathrm{Ge} \quad \mathrm{p}$-Channel Metal-Oxide-Semiconductor Field-Effect Transistors Fabricated Using Thermally Oxidized $\mathrm{GeO}_{2}$ Interfacial Layers, Jpn. J. Appl. Phys. 2011;50:010109.

[201] D. Kuzum, J.-H. Park, T. Krishnamohan, H. S-.P. Wong, and K. C. Saraswat, The effect of donor/acceptor nature of interface traps on Ge MOSFET characteristics, IEEE Trans. Electron Devices 2011;58:1015.

[202] K. Hirayama, R. Ueno, Y. Iwamura, K. Yoshino, D. Wang, H. Yang, and H. Nakashima, Fabrication of Ge Metal-Oxide-Semiconductor Capacitors with High-Quality Interface by Ultrathin $\mathrm{SiO}_{2} / \mathrm{GeO}_{2}$ Bilayer Passivation and Postmetallization Annealing Effect of Al, Jpn. J. Appl. Phys. 2011;50:04DA10.

[203] A. Toriumi, C. H. Lee, S. K. Wang, T. Tabata, M. Yoshida, D. D. Zhao, T. Nishimura, K. Kita, and K. Nagashio, Material Potential and Scalability Challenges of Germanium CMOS, In: IEDM Tech. Dig. 2011; p. 646-649.

[204] R. Zhang, T. Iwasaki, N. Taoka, M. Takenaka, and S. Takagi, $\mathrm{Al}_{2} \mathrm{O}_{3} / \mathrm{GeO}_{x} / \mathrm{Ge}$ gate stacks with low interface trap density fabricated by electron cyclotron resonance plasma postoxidation, Appl. Phys. Lett. 2011;98:112902.

[205] R. Zhang, T. Iwasaki, N. Taoka, M. Takenaka, and S. Takagi, High Mobility Ge pMOSFETs with $\sim 1 \mathrm{~nm}$ Thin EOT Using $\mathrm{Al}_{2} \mathrm{O}_{3} / \mathrm{GeO}_{x} / \mathrm{Ge}$ Gate Stacks Fabricated by Plasma Post Oxidation, In: Symp. VLSI Tech. Dig. 2011; p. 56-57. 
[206] R. Zhang, T. Iwasaki, N. Taoka, M. Takenaka and S. Takagi, Impact of $\mathrm{GeO}_{\mathrm{x}}$ interfacial layer thickness on $\mathrm{Al}_{2} \mathrm{O}_{3} / \mathrm{Ge}$ MOS interface properties, Microelectron. Eng. $2011 ; 88: 1533$.

[207] R. Zhang, P. C. Huang, N. Taoka, M. Takenaka, and S. Takagi, High Mobility Ge pMOSFETs with $0.7 \mathrm{~nm}$ Ultrathin EOT using $\mathrm{HfO}_{2} / \mathrm{Al}_{2} \mathrm{O}_{3} / \mathrm{GeO}_{\mathrm{x}} / \mathrm{Ge}$ Gate Stacks Fabricated by Plasma Post Oxidation, In: Symp. VLSI Tech. Dig. 2012; p. 161-162.

[208] A. Wada, R. Zhang, S. Takagi, and S. Samukawa, High-quality germanium dioxide thin films with low interface state density using a direct neutral beam oxidation process, Appl. Phys. Lett. 2012;100: 213108.

[209] S. Takagi, R. Zhang, and M. Takenaka, Ge gate stacks based on Ge oxide interfacial layers and the impact on MOS device properties, Micoroelectron. Eng. 2013;109:389 .

[210] K. Ikeda, Y. Kamimuta, Y. Moriyama, M. Ono, K. Usuda, M. Oda, T. Irisawa, K. Furuse, and T. Tezuka, Enhancement of Hole Mobility and Cut-Off Characteristics of Strained Ge Nanowire pMOSFETs by Using Plasma Oxidized GeOx Inter-Layer for Gate Stack, In: Symp. VLSI Tech. Dig. 2013; p. T30-T31.

[211] Y. Minoura, A. Kasuya, T. Hosoi, T. Shimura, and H. Watanabe, Design and control of Ge-based metal-oxide-semiconductor interfaces for high-mobility field-effect transistors with ultrathin oxynitride gate dielectrics, Appl. Phys. Lett. 2013;103:033502. [212] R. Zhang, W. Chern, X. Yu, M. Takenaka, J. L. Hoyt, and S. Takagi, High Mobility Strained-Ge pMOSFETs with 0.7-nm Ultrathin EOT using Plasma Post Oxidation $\mathrm{HfO}_{2} / \mathrm{Al}_{2} \mathrm{O}_{3} / \mathrm{GeO}_{\mathrm{x}}$ Gate Stacks and Strain Modulation, In: IEDM Tech. Dig. 2013; p. 633-636.

[213] C. Lu, C.-H. Lee, W. Zhang, T. Nishimura, K. Nagashio, and A. Toriumi, 
Enhancement of thermal stability and water resistance in yttrium-doped $\mathrm{GeO}_{2} / \mathrm{Ge}$ gate stack, Appl. Phys. Lett. 2014;104:092909.

[214] K. Tanaka, R. Zhang, M. Takenaka, and S. Takagi, Quantitative evaluation of slow traps near Ge MOS interfaces by using time response of MOS capacitance, Jpn. J. Appl. Phys. 2015;54: 04DA02.

[215] C. Lu and A. Toriumi, Structural coordination of rigidity with flexibility in gate dielectric films for sub-nm EOT Ge gate stack reliability, In: IEDM Tech. Dig. 2015; p. 370-373.

[216] M. Ke, X. Yu, R. Zhang, J. Kang, C. Chang, M. Takenaka and S. Takagi, Fabrication and MOS interface properties of $\mathrm{ALD} \mathrm{AlYO}_{3} / \mathrm{GeO}_{\mathrm{x}} / \mathrm{Ge}$ gate stacks with plasma post oxidation, Microelectron. Eng. 2015;147:244-248.

[217] N. Wu, Q. Zhang, C. Zhu, D. S. H. Chan, M. F. Li, N. Balasubramanian, A. Chin, D. L. Kwong, Alternative surface passivation on germanium for metal-oxide-semiconductor applications with high-k gate dielectric, Appl. Phys. Lett. $2004 ; 85: 4127$.

[218] B. De Jaeger, R. Bonzom, F. Leys, O. Richard, J. Van Steenbergen, G. Winderickx, E. Van Moorhem, G. Raskin, F. Letertre, T. Billon, M. Meuris, M. Heyns, Optimisation of a thin epitaxial Si layer as Ge passivation layer to demonstrate deep sub-micron nand p-FETs on Ge-On-Insulator substrates, Microelectron. Eng. 2005;80:26.

[219] P. Zimmarman, G. Nicholas, B. De Jaeger, B. Kaczer, A. Stesmans, L.-A. Rangnarsson, D. P. Brunco, F. E. Leys, M. Caymax, G. Winderickx, K. Opsomer, M. Meuris, M. M. Heyns, High performance Ge pMOS devices using a Si-compatible process flow, In: IEDM Tech. Dig. 2006; p. 655-658.

[220] T. Yamamoto, Y. Yamashita, M. Harada, N. Taoka, K. Ikeda, K. Suzuki, O. Kiso, 
N. Sugiyama, S. Takagi, High Performance $60 \mathrm{~nm}$ Gate Length Germanium p-MOSFETs with Ni Germanide Metal Source/Drain, In: IEDM Tech. Dig. 2007; p. 1041-1043.

[221] N. Taoka, M. Harada, Y. Yamashita, T. Yamamoto, N. Sugiyama, S. Takagi, Effects of Si passivation on Ge metal-insulator-semiconductor interface properties and inversion-layer hole mobility, Appl. Phys. Lett 2008;92:113511.

[222] N. Taoka, W. Mizubayashi, Y. Morita, S. Migita, H. Ota and S. Takagi, Physical Origins of Mobility Enhancement of Ge p-channel Metal-Insulator-Semiconductor Field Effect Transistors with Si Passivation Layers, J. Appl. Phys. 2010;108:104511.

[223] R. Zhang, J.-C. Lin, X. Yu, M. Takenaka, and S. Takagi, Impact of plasma post oxidation temperature on interface trap density and roughness at $\mathrm{GeO}_{\mathrm{x}} / \mathrm{Ge}$ interfaces, Micoroelectron. Eng. 2013;109:97.

[224] N. Taoka, M. Yokoyama, S. H. Kim, R. Suzuki, R. Iida, S. Lee, T. Hoshii, W. Jevasuwan, T. Maeda, T. Yasuda, O. Ichikawa, N. Fukuhara, M. Hata, M. Takenaka and S. Takagi, Impact of Fermi Level Pinning inside Conduction Band on Electron Mobility of $\mathrm{In}_{\mathrm{x}} \mathrm{Ga}_{1-\mathrm{x}} \mathrm{As}$ MOSFETs and Mobility Enhancement by Pinning Modulation, In: IEDM Tech. Dig. 2011; p. 610-614.

[225] N. Taoka, M. Yokoyama, S.-H. Kim, R. Suzuki, S.-H. Lee, R. Iida, T. Hoshii, W Jevasuwan, T. Maeda, T. Yasuda, O. Ichikawa, N. Fukuhara, M. Hata, M. Takenaka and S. Takagi, Impact of Fermi Level Pinning inside Conduction Band on Electron Mobility of InGaAs MOSFETs, Appl. Phys. Lett. 2013;103:143509.

[226] N. Taoka, M. Yokoyama, S.-H. Kim, R. Suzuki, S.-H. Lee, R. Iida, T. Hoshii, W. Jevasuwan, T. Maeda, T. Yasuda, O. Ichikawa, N. Fukuhara, M. Hata, M. Takenaka and S. Takagi, Impact of Fermi Level Pinning due to Interface Traps inside Conduction 
Band on Inversion-layer Mobility of InxGa1-xAs Metal-Oxide-Semiconductor Field Effect Transistors, IEEE Transactions on Device and Materials Reliability 2013;13:456-462.

[227] T.-J. King Liu, K. J. Kuhn, CMOS and Beyond: Logic Switches for Terascale Integrated Circuits, Cambridge University Press, Cambridge, 2014

[228] D. K. Mohata, R. Bijesh, S. Mujumdar, C. Eaton, R. Engel-Herbert, T. Mayer, V. Narayanan, J. M. Fastenau, D. Loubychev, A. K. Liu and S. Datta, Demonstration of MOSFET-like on-current performance in arsenide/antimonide tunnel FETs with staggered hetero-junctions for 300mV logic applications, In: IEDM Tech. Dig. 2011; p. 781-784.

[229] G. Zhou, R. Li, T. Vasen, M. Qi, S. Chae, Y. Lu, Q. Zhang, H. Zhu, J.-M. Kuo, T. Kosel, M. Wistey, P. Fay, A. Seabaugh and H. Xing, Novel gate-recessed vertical InAs/GaSb TFETs with record high Ion of $180 \mu \mathrm{A} / \mu \mathrm{m}$ at $\mathrm{V}_{\mathrm{DS}}=0.5 \mathrm{~V}$, In: IEDM Tech. Dig. 2012; p. 777-780.

[230] A. W. De, B. M. Borgt, B. Ganjipourt, M. Ek, K. A. Dick, E. Lind, P. Nilsson, C. Thelander and L.-E. Wernersson, High current density InAsSb/GaSb tunnel field effect transistors, In: Proc. IEEE Device Research Conf.; 2012, p. 205-206.

[231] R. Li, Y. Lu, G. Zhou, Q. Liu, S. D. Chae, T. Vasen, W. S. Hwang, Q. Zhang, P. Fay, T. Kosel, M. Wistey, H. Xing and A. Seabaugh, AlGaSb/InAs tunnel field-effect transistor with on-current of $78 \mu \mathrm{A} / \mu \mathrm{m}$ at $0.5 \mathrm{~V}$, IEEE Electron Device Lett. 2012;33: $363-365$.

[232] R. Bijesh, H. Liu, H. Madan, D. Mohata, W. Li, N. V. Nguyen, D. Gundlach, C. A. Richter, J. Maier, K. Wang, T. Clarke, J. M. Fastenau, D. Loubychev, W. K. Liu, V. Narayanan and S. Datta, Demonstration of $\operatorname{In}_{0.9} \mathrm{Ga}_{0.1} \mathrm{As}_{\mathrm{s}} / \mathrm{GaAs}_{0.18} \mathrm{Sb}_{0.82}$ near broken-gap 
tunnel FET with $\mathrm{ION}=740 \mu \mathrm{A} / \mu \mathrm{m}, \mathrm{G}_{\mathrm{M}}=700 \mu \mathrm{S} / \mu \mathrm{m}$ and gigahertz switching performance at $\mathrm{V}_{\mathrm{DS}}=0.5 \mathrm{~V}$, In: IEDM Tech. Dig. 2013; p. 687-690.

[233] M. Noguchi, S.-H. Kim, M. Yokoyama, O. Ichikawa, T. Osada, M. Hata, M. Takenaka, and S. Takagi, High Ion/Ioff and low subthreshold slope planar-type InGaAs Tunnel FETs with Zn-diffused source junctions, In: IEDM Tech. Dig. 2013; p. 683-683. [234] M. Noguchi, S.-H. Kim, M. Yokoyama, O. Ichikawa, T. Osada, M. Hata, M. Takenaka and S. Takagi, High $\mathrm{I}_{\text {on }} / \mathrm{I}_{\text {off }}$ and low subthreshold slope planar-type InGaAs tunnel field effect transistors with Zn-diffused source junctions, J. Appl. Phys. 2015; 118:045712.

[235] M.-S. Kim, Y.-H Kim, M. Yokoyama, R. Nakane, S.-H. Kim, M. Takenaka and S. Takagi, Tunnel Field-Effect Transistors with Germanium/Strained-Silicon Hetero-junctions for Low Power Application, Thin Solid Films 2014;557:298-301.

[236] M.-S. Kim, Y. Wakabayashi, R. Nakane, M. Yokoyama, M. Takenaka and S. Takagi, High Ion/Ioff Ge-source ultrathin body strained-SOI Tunnel FETs - impact of channel strain, MOS interfaces and back gate on the electrical properties", In: IEDM Tech. Dig. 2014; p. 331-334.

[237] Y. Yamamoto and H. Kanbe, Zn Diffusion in $\operatorname{In}_{x} \mathrm{Ga}_{1-\mathrm{x}} \mathrm{As}$ with ZnAs2 Source, Jpn. J. Appl. Phys. 1980;19:121-128.

[238] B. Tuck, Atomic Diffusion in III-V Semiconductors, p. 75, Adam Hilger, Bristol and Philadelphia, 1988.

[239] R. Iida, S.-H. Kim, M. Yokoyama, N. Taoka, S.-H. Lee, M. Takenaka and S. Takagi, Planar-type In $0.53 \mathrm{Ga} 0.47 \mathrm{As}$ channel band-to-band tunneling Metal-Oxide-Semiconductor Field-Effect Transistors, J. Appl. Phys. 2011;110:124505. [240] A. Alian, J. Franco, A. Vandooren, Y. Mols, A. Verhulst, S. El Kazzi, R. 
Rooyackers, D. Verreck, Q. Smets, A. Mocuta, N. Collaert, D. Lin, and A. Thean, "Record performance InGaAs homo-junction TFET with superior SS reliability over MOSFET”, In: IEDM Tech. Dig. 2015; p. 823-826.

[241] H. Riel, K. E. Moselund, C. Bessire, M. T. Björk, A. Schenk, H. Ghoneim, H. Schmid, InAs-Si Heterojunction Nanowire Tunnel Diodes and Tunnel FETs, In: IEDM Tech. Dig. 2012; p. 391-394.

[242] K. Tomioka, M. Yoshimura, and T. Fukui, Sub $60 \mathrm{mV} /$ decade Switch Using an InAs Nanowire-Si Heterojunction and Turn-on Voltage Shift with a Pulsed Doping Technique, Nano Lett. 2013;13:5822-5826.

[243] Q. T. Zhao, J. M. Hartmann and S. Mantl, An Improved Si Tunnel Field Effect Transistor with a Buried Strained $\mathrm{Si}_{1-\mathrm{x}} \mathrm{Ge}_{\mathrm{x}}$ Source, IEEE Electron Device Lett. 2011;32:1480-1482.

[244] Q. T. Zhao, W. J. Yu, B. Zhang, M. Schmidt, S. Richter, D. Buca, J.-M. Hartmann, R. Luptak, A. Fox, K. K. Bourdelle, and S. Mantl, Tunneling field-effect transistor with a strained Si channel and a Si0.5Ge0.5 source, Solid-State Electronics 2012;74:97-101.

[245] L. Knoll, Q. T. Zhao, A. Nichau, S. Richter, G.V. Luong, S. Trellenkamp, A. Schäfer, L. Selmi, K. K. Bourdelle, and S. Mantl, Demonstration of Improved Transient Response of Inverters with Steep Slope Strained Si NW TFETs by Reduction of TAT with Pulsed I-V and NW Scaling, In: IEDM Tech. Dig. 2013; p. 100-104.

[246] T. Hoshii, S. Sugahara and S. Takagi, Effect of Tensile Strain on Gate Current of Strained-Si n-Channel Metal-Oxide-Semiconductor Field-Effect Transistors, Jpn. J. Appl. Phys. 2007;46;2122-2126.

[247] M.-S. Kim, Y. Wakabayashi, M. Yokoyama, R. Nakane, M. Takenaka and S. Takagi, Electrical Characteristics of $\mathrm{Ge} / \mathrm{Si}$ Hetero-Junction Tunnel Field-Effect 
Transistors and Their Post Metallization Annealing Effect, IEEE Trans. Electron Devices 2015;62:9-14.

[248] W. Y. Choi, B.-G. Park, J. D. Lee, and T.-J. King Liu, Tunneling Field-Effect Transistors (TFETs) With Subthreshold Swing (SS) Less Than $60 \mathrm{mV} / \mathrm{dec}$, IEEE Electron Device Lett. 2007; 28:743-745.

[249] T. Krishnamohan, D. Kim, S. Raghunathan, K. Saraswat, Double-Gate Strained-Ge Heterostructure Tunneling FET (TFET) With Record High Drive Currents and $<60 \mathrm{mV} /$ dec subthreshold Slope, In: IEDM Tech. Dig. 2008; p. 947-950.

[250] G. Han, P. Guo, Y. Yang, C. Zhan, Q. Zhou and Y.-C. Yeo, Silicon-based tunneling field-effect transistor with elevated germanium source formed on (110) silicon substrate, Appl. Phys. Lett. 2011;98:153502.

[251] W.-Y. Loh, K. Jeon, C. Y. Kang, J. Oh, T.-J. K. Liu, H.-H. Tseng, W. Xiong, P. Majhi, R. Jammy and C. Hu, Highly scaled $(\mathrm{Lg} \sim 56 \mathrm{~nm})$ gate-last Si tunnel field effect transistors with Ion $>100 \mathrm{~mA} / \mathrm{mm}$, Solid-State Electron. 2011;65-66:22-27.

[252] G. Dewey, B. Chu-Kung, J. Boardman, J. M. Fastenau, J. Kavalieros, R. Kotlyar, W. K. Liu, D. Lubyshev, M. Metz, N. Mukherjee, P. Oakey, R. Pillarisetty, M. Radosavljevic, H. W. Then, and R. Chau, Fabrication, Characterization, and Physics of III-V Heterojunction Tunneling Field Effect Transistors (H-TFET) for Steep Sub-Threshold Swing, In: IEDM Tech. Dig. 2011; p. 785-788.

[253] Q. Huang, R. Huang, Z. Zhan, Y. Qiu, W. Jiang, C. Wu, Y. Wang, A Novel Si Tunnel FET with $36 \mathrm{mV} / \mathrm{dec}$ subthreshold Slope Based on Junction Depleted-Modulation through Striped Gate Configuration, In: IEDM Tech. Dig. 2012; p. 187-190.

[254] M. H. Lee, J.-C. Lin, and C.-Y. Kao, Hetero-Tunnel Field-Effect-Transistors With 
Epitaxially Grown Germanium on Silicon, IEEE Trans. Electron Devices 2013;60: 2423-2327. 


\section{FIGURE CAPTIONS}

Fig. 1: Two possible strategies to further reduce $V d d$.

Fig. 2: Possible structures for advanced MOSFETs and TFETs using III-V/Ge devices on Si platform through heterogeneous integration

Fig. 3: Critical issues for realizing III-V/Ge MOSFETs and TFETs on the Si platform

Fig. 4: (a) Fabrication process of GaSb-OI substrates (b) Cross-sectional TEM images of 23-nm-thick GaSb-OI on a Si wafer

Fig. 5: (a) Fabrication process of InGaAs-OI wafer by direct wafer bonding technique using InGaAs on a $\mathrm{Si}$ donor wafer. $\mathrm{InGaAs} / \mathrm{In}_{x} \mathrm{Al} 1_{1-\mathrm{x}} \mathrm{As} / \mathrm{GaAs}$ layers are epitaxially grown on $\mathrm{Si}$ substrates. After $\mathrm{Al}_{2} \mathrm{O}_{3}$ deposition as a $\mathrm{BOX}$ layer, $\mathrm{CMP}$ has been carried out for surface smoothing for $\mathrm{Al}_{2} \mathrm{O}_{3} / \mathrm{III}-\mathrm{V} / \mathrm{Si}$ wafer and wafers were bonded each other. Subsequent wet etching thinned the top Si and the III-V buffer layers, resulting in the formation of InGaAs-OI on Si substrates. (b) Cross-sectional TEM image of the bonded $\mathrm{Si} / \mathrm{III}-\mathrm{V} / \mathrm{BOX} / \mathrm{Si}$ wafer before top $\mathrm{Si}$, III-V buffer etching. (c) Cross-sectional TEM image of InGaAs-OI on Si after top Si, III-V buffer etching. A high resolution image is also shown.

Fig. 6: (a) Measured $I_{d}-V_{g}$ characteristics of InGaAs-OI MOSFETs using an InGaAs layer on a Si donor wafer (b) $\mu_{\text {eff-T }} T_{\text {body }}$ characteristics of InGaAs-OI MOSFETs on the wafer from an InP and a $\mathrm{Si}$ donor wafer (c) Cumulative distribution of the leakage current at $L_{G}$ of $1 \mu \mathrm{m}$ and $V_{D}$ of $50 \mathrm{mV}$.

Fig. 7: $C$ - $V$ curves of (a) $\mathrm{Al}_{2} \mathrm{O}_{3}(3.5 \mathrm{~nm}) / \mathrm{InGaAs}$, (b) $\mathrm{Al}_{2} \mathrm{O}_{3}(3.5 \mathrm{~nm}) / \mathrm{La}_{2} \mathrm{O}_{3}(0.4 \mathrm{~nm}(10$ cycles))/InGaAs and (c) $\mathrm{Al}_{2} \mathrm{O}_{3}(3.5 \mathrm{~nm}) / \mathrm{La}_{2} \mathrm{O}_{3}(2.9 \mathrm{~nm}$ (40 cycles))/InGaAs MOS gate stacks with Au gate electrodes 
Fig. 8: $D_{i t}$ of $\mathrm{Al}_{2} \mathrm{O}_{3}(3.5 \mathrm{~nm}) / \mathrm{La}_{2} \mathrm{O}_{3} / \mathrm{InGaAs}$ at the surface energy of $0.1 \mathrm{eV}$ from the midgap as a function of the $\mathrm{La}_{2} \mathrm{O}_{3}$ ALD cycle number, corresponding to the change in the $\mathrm{La}_{2} \mathrm{O}_{3}$ thickness from $0 \mathrm{~nm}$ to $2.9 \mathrm{~nm}$. Here, data of $\mathrm{La}_{2} \mathrm{O}_{3}(2.9 \mathrm{~nm}$ (40 cycles))/ InGaAs MOS gate stacks without $\mathrm{Al}_{2} \mathrm{O}_{3}$ has also been added

Fig. 9: $C$ - $V$ curves of the $\mathrm{Al}_{2} \mathrm{O}_{3} / \mathrm{InAs} / \mathrm{p}-\mathrm{GaSb}$ MOS capacitors with the InAs thickness of (a) 0 , (b) 0.5 , (c) 1.5 and (d) $3 \mathrm{~nm}$, measured at room temperature.

Fig. 10: Energy distribution of $D_{i t}$ of the $\mathrm{Al}_{2} \mathrm{O}_{3} / \mathrm{InAs} / \mathrm{p}-\mathrm{GaSb}$ MOS capacitors with the InAs thickness of $0,0.5,1.0,1.5$, and $2.0 \mathrm{~nm}$. The surface energy is taken from the midgap energy of GaSb. Here, $D_{i t}$ was evaluated from the $1 \mathrm{MHz} C-V$ curves at $100 \mathrm{~K}$ by using the Terman method.

Fig. 11: Device structure and the schematic process flow of InGaAs-OI tri-gate MOSFETs using In ${ }_{0.3} \mathrm{Ga}_{0.7} \mathrm{As}(3 \mathrm{~nm}) / \mathrm{InAs}(3 \mathrm{~nm}) / \mathrm{In}_{0.3} \mathrm{Ga}_{0.7} \mathrm{As}(3 \mathrm{~nm})-\mathrm{OI}$ QW channels. A top view Scanning Electron Microscope (SEM) photograph of a tri-gate MOSFET with a narrow channel width is also shown.

Fig. 12 (a) $I_{d-} V_{g}$ characteristics of the In0.3 Ga0.7As/InAs/In0.3Ga0.7As-OI tri-gate MOSFETs with $L_{c h}$ of $20 \mathrm{~nm}$ as a parameter of $\mathrm{W}$ of 40, 50, 140, and $360 \mathrm{~nm}$ (b) DIBL characteristics with different $W_{G}$ as a function of $L_{c h}$ (c) $I_{o n}$ at $V_{D D}$ of $0.5 \mathrm{~V}$ and $I_{o f f}$ of $100 \mathrm{nA} / \mu \mathrm{m}$ characteristics with different $W_{G}$ as a parameter of $L_{c h}$

Fig. 13: Benchmark of $I_{o n}$ at $V_{d}$ of $0.5 \mathrm{~V}$ with gate overdrive of $0.5 \mathrm{~V}$, taken from the present $\mathrm{In}_{0.3} \mathrm{Ga}_{0.7} \mathrm{As} / \mathrm{InAs} / \mathrm{In}_{0.3} \mathrm{Ga}_{0.7} \mathrm{As}-\mathrm{OI}$ tri-gate MOSFETs and reported advanced InGaAs MOSFETs. Here, $V_{g}$ has been shifted so that $I_{\text {off }}$ at gate overdrive of $0 \mathrm{~V}$ amounts to $100 \mathrm{nA} / \mu \mathrm{m}$.

Fig. 14: Proposed $\mathrm{Al}_{2} \mathrm{O}_{3} / \mathrm{GeO}_{x} / \mathrm{Ge}$ and $\mathrm{HfO}_{2} / \mathrm{Al}_{2} \mathrm{O}_{3} / \mathrm{GeO}_{\mathrm{x}} / \mathrm{Ge}$ gate stack formation process by oxidation using ECR oxygen plasma through a thin $\mathrm{Al}_{2} \mathrm{O}_{3}$ and a thin 
$\mathrm{HfO}_{2} / \mathrm{Al}_{2} \mathrm{O}_{3}$ layer, respectively, by ALD

Fig. 15: EOT and $D_{i t}$ of the $\mathrm{Au} / \mathrm{HfO}_{2}(2.2 \mathrm{~nm}) / \mathrm{Al}_{2} \mathrm{O}_{3}(0.2 \mathrm{~nm}) / \mathrm{GeO}_{\times} / \mathrm{Ge}$ MOS capacitors with different plasma post oxidation times at $0.2 \mathrm{eV}$ from the midagap

Fig. 16: (a) Hysteresis in forward and backward scan sweep of $I_{d}-V_{g}$ characteristics of Ge n- and p-MOSFETs with TiN/ $/ \mathrm{Al}_{2} \mathrm{O}_{3} / \mathrm{GeO}_{\mathrm{x}} / \mathrm{Ge}$ gate stacks (b) time dependence of $I_{d}$ of the Ge n- and p-MOSFETs with $\mathrm{GeO}_{\mathrm{x}}$ thickness of 0.27 and $1.2 \mathrm{~nm}$ at constant $V_{d}$ and $V_{g}$ values

Fig. 17: $C$ - $V$ curves of (a) 1.5-nm-thick $\mathrm{Al}_{2} \mathrm{O}_{3} / \mathrm{GeO}_{\mathrm{x}} / \mathrm{Ge}$ with plasma post oxidation, (b) 1.5-nm-thick $\mathrm{AlYO}_{3} / \mathrm{Ge}$ MOS capacitors with plasma post oxidation and (c) 1.5-nm-thick $\mathrm{AlYO}_{3} / \mathrm{GeO}_{x} / \mathrm{Ge}$ MOS capacitors with plasma post oxidation. The measurement frequency was varied from $1 \mathrm{~K}, 10 \mathrm{~K}, 100 \mathrm{~K}$ to $1 \mathrm{MHz}$.

Fig. 18: (a) Energy distributions of $D_{i t}$ at 1.5-nm-thick $\mathrm{AlYO}_{3} / \mathrm{Ge}$ MOS interfaces with and without plasma post oxidation (b) Relationship between $E_{o x}$ and $\Delta N_{f i x}$ of 1.5-nm-thick $\mathrm{AlYO}_{3} / \mathrm{GeO}_{x} / \mathrm{p}-\mathrm{Ge}$ and n-Ge $\mathrm{MOS}$ capacitors and 1.5-nm-thick $\mathrm{Al}_{2} \mathrm{O}_{3} / \mathrm{GeO}_{x} / \mathrm{n}-\mathrm{Ge}$ and $\mathrm{p}-\mathrm{Ge}$ MOS capacitors.

Fig. 19: $I_{d-} V_{g}$ characteristics of (100) Ge p- and n-MOSFET with the $\mathrm{HfO}_{2} / \mathrm{Al}_{2} \mathrm{O}_{3} / \mathrm{GeO}_{\mathrm{x}} / \mathrm{Ge}$ gate stack having an EOT of $0.78 \mathrm{~nm}$

Fig. 20: (a) Effective and Hall electron mobility of $\mathrm{Al}_{2} \mathrm{O}_{3} / \mathrm{GeO}_{\mathrm{x}} / \mathrm{Ge}$ n-MOSFETs with $\mathrm{N}_{2}$ and atomic deuterium PDA in comparison with the effective mobility reported previously $[159,160,168]$ (b) $D_{i t}$ inside the conduction band, evaluated by Hall measurements for $\mathrm{Al}_{2} \mathrm{O}_{3} / \mathrm{GeO}_{x} / \mathrm{Ge}$ n-MOSFETs with PDA in $\mathrm{N}_{2}$ and atomic deuterium ambient.

Fig. 21: Device/material engineering for solving these critical issues and challenges of p-n junction-type TFETs 
Fig. 22: (a) Schematic band lineup of the type-II hetero-structure and (b) band diagram of the source p-n junction region composed of the type-II hetero-structure.

Fig. 23: Calculated $\mathrm{Zn}$ profiles under the three diffusion constant models, where diffusion coefficients are constant, proportional to $[\mathrm{Zn}]$ and proportional to $[\mathrm{Zn}]^{2}$ ones. Fig. 24: (a) SIMS profiles of diffused $\mathrm{Zn}$ and implanted Be atoms in InGaAs. Here, Zn was diffused at $500{ }^{\circ} \mathrm{C}$ for 1 minute in a $\mathrm{N}_{2}$ ambient. Be was introduced by $\mathrm{Be}$ implantation with the energy of $2 \mathrm{keV}$ and the dose of $1 \times 10^{15} \mathrm{~cm}^{-2}$. Also, the solid curve shows the profile calculated using the model that the diffusion constant of $\mathrm{Zn}$ in InGaAs is proportional to the square of the $\mathrm{Zn}$ concentration. (b) $I-V$ characteristics of Zn-diffused, Zn-implanted and Be-implanted $\operatorname{In}_{0.53} \mathrm{Ga}_{0.47} \mathrm{As}^{+}$-n junctions.

Fig. 25: (a) $I_{d-} V_{g}$ characteristics at $V_{d}$ of $150 \mathrm{mV}$ of an $\operatorname{In}_{0.53} \mathrm{Ga} 0.47 \mathrm{As}$ TFET with source regions formed by $\mathrm{Zn}$ diffusion at $500{ }^{\circ} \mathrm{C}$ for $1 \mathrm{~min}$. Inset is the schematic structure of the $\operatorname{In}_{0.53} \mathrm{Ga}_{0.47} \mathrm{As}$ TFET (b) $I_{d^{-}} V_{d}$ characteristics of the $\operatorname{In}_{0.53} \mathrm{Gaa}_{0.47} \mathrm{As}$ TFET.

Fig. 26: Temperature dependence of $I_{d}-\left(V_{g}-V_{t h}\right)$ characteristics at $V_{d}$ of $150 \mathrm{mV}$ of an InGaAs TFET with the source region formed by $\mathrm{Zn}$ diffusion at $500{ }^{\circ} \mathrm{C}$ for $1 \mathrm{~min}$ from 6 $\mathrm{K}, 50 \mathrm{~K}, 171 \mathrm{~K}, 227 \mathrm{~K}, 254 \mathrm{~K}$ to $292 \mathrm{~K}$. Here, $\mathrm{V}_{\text {th }}$ is defined as $V_{g}$ at $I_{d}$ of $10^{-7} \mathrm{~A} / \mu \mathrm{m}$.

Fig. 27: (a) Schematic band diagram of the hetero-interface of Ge and tensile strain Si (b) fabricated Ge/strained Si TFET structure

Fig. 28: $I_{d}-V_{d}$ characteristics of Ge-source TFETs fabricated on un-strained SOI, 0.8 and $1.1 \%$ strained SOI substrates

Fig. 29: Benchmark of the present n-channel TFET performance in terms of $I_{\text {on }} / I_{\text {off }}$ ratio versus the minimum S.S. value with other reported ones [18-20, 243, 248-254] with a variety of channel materials 


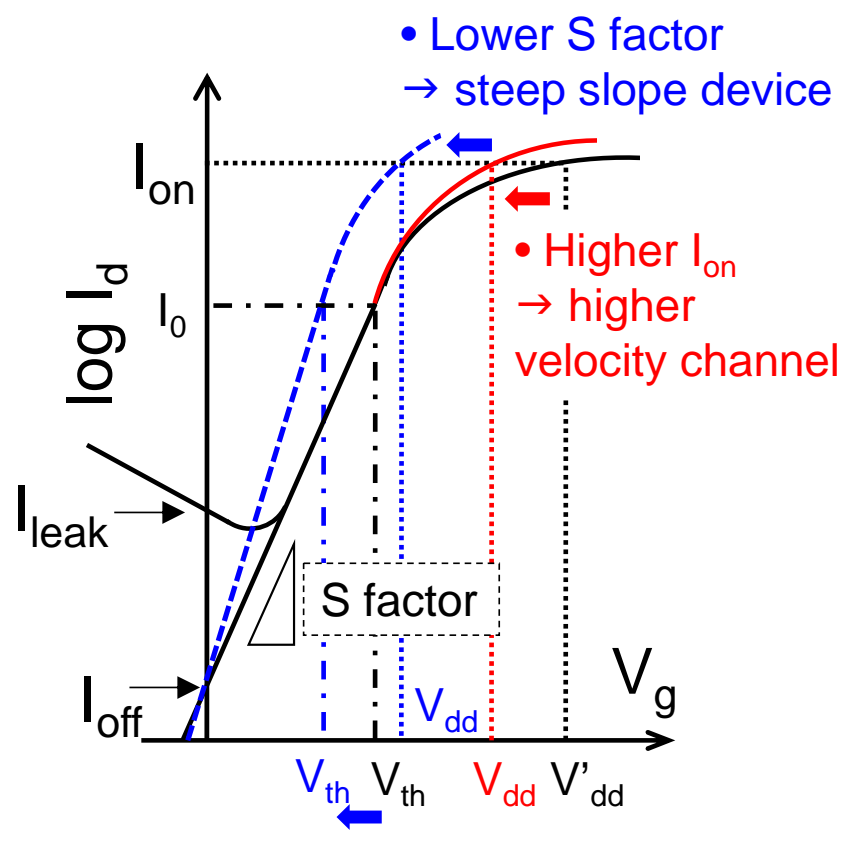

Fig. 1 (S. Takagi)

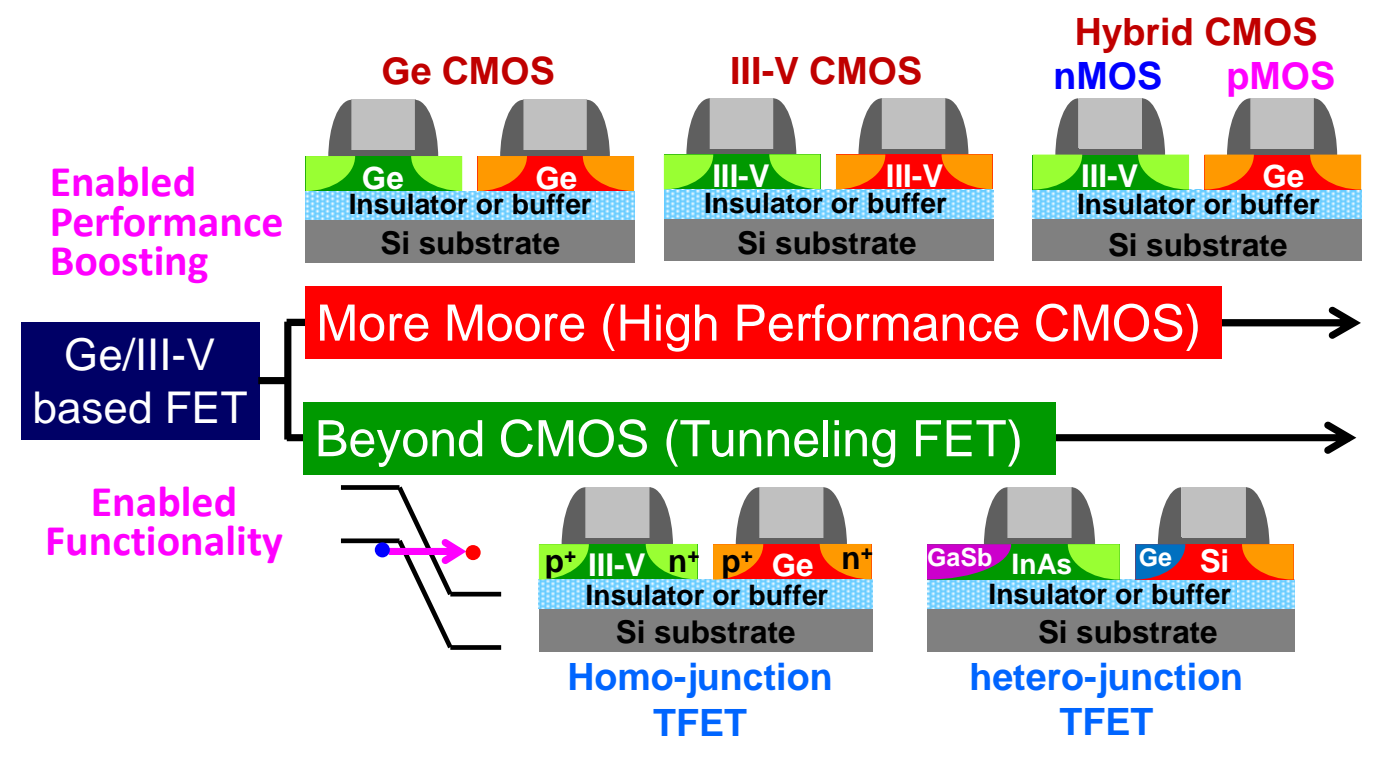

Fig. 2 (S. Takagi) 
- MOS gate stack with low $D_{\text {it }}$ $\checkmark$ thin EOT with low defects $\checkmark$ high channel mobility $\checkmark$ high gate stack reliability including long term $\mathrm{V}_{\text {th }}$ stability

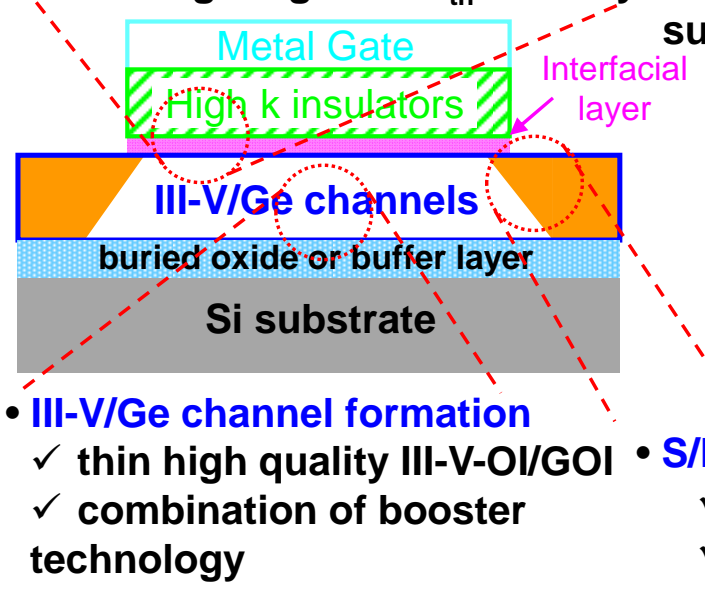

Fig. 3 (S. Takagi)
- Optimum channel design $\checkmark$ UTB, Fin or nano-wire channel formation $\checkmark$ uniformity of channel thickness/radius and small surface roughness - CMOS integration technology $\checkmark$ compatibility with $\mathrm{Si}$ CMOS platform $\checkmark$ appropriate choice of $\mathrm{n}$ - and $\mathrm{p}$-MOSFET $\because$ integration process S/D formation technology $\checkmark$ low resistance S/D $\checkmark$ low junction leakage 


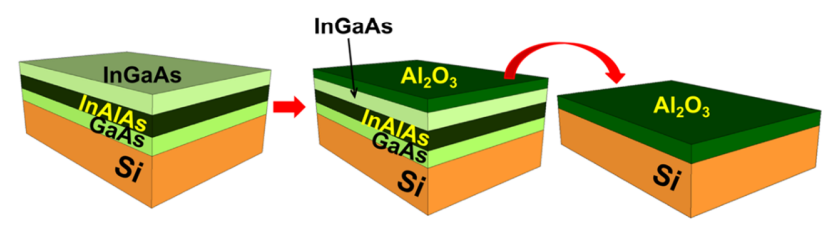

(a)

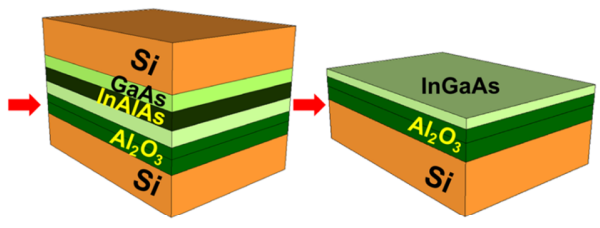

(b)

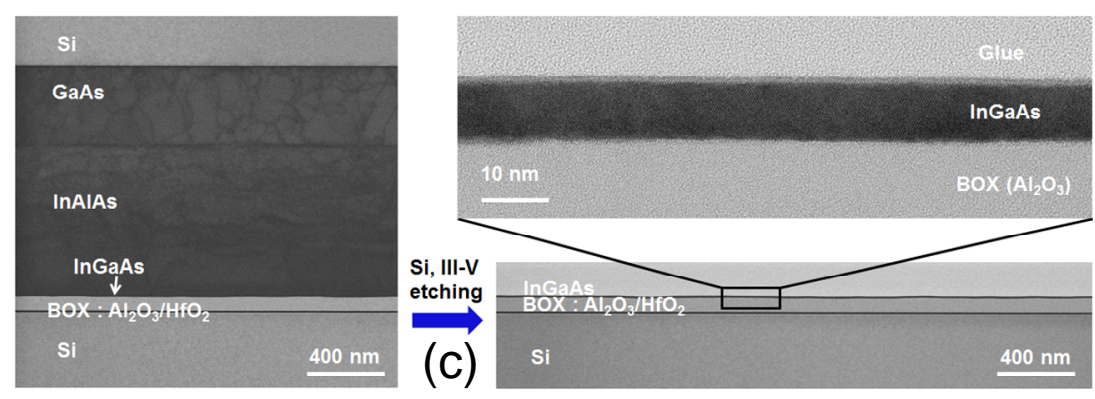

Fig. 5 (S. Takagi)
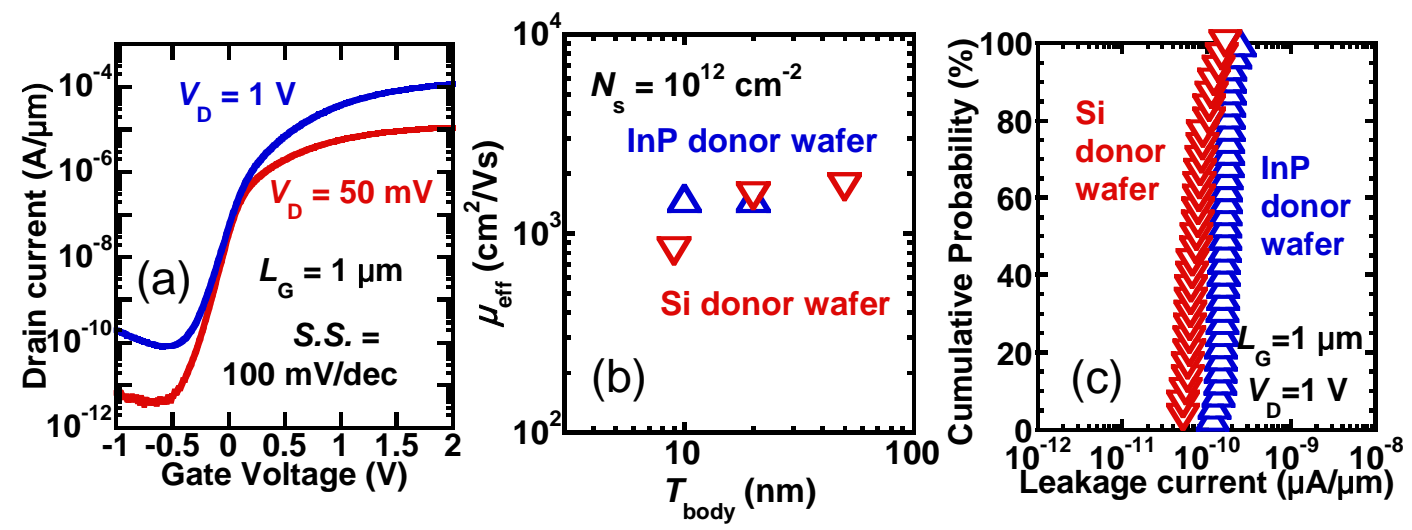

Fig. 6 (S. Takagi) 

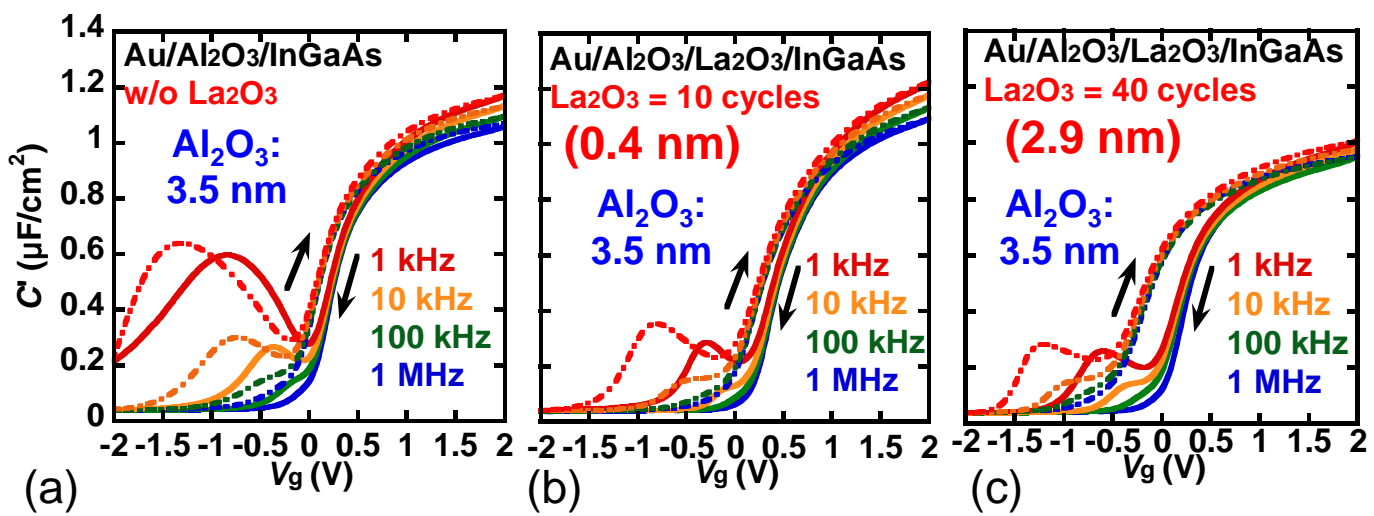

Fig. 7 (S. Takagi)

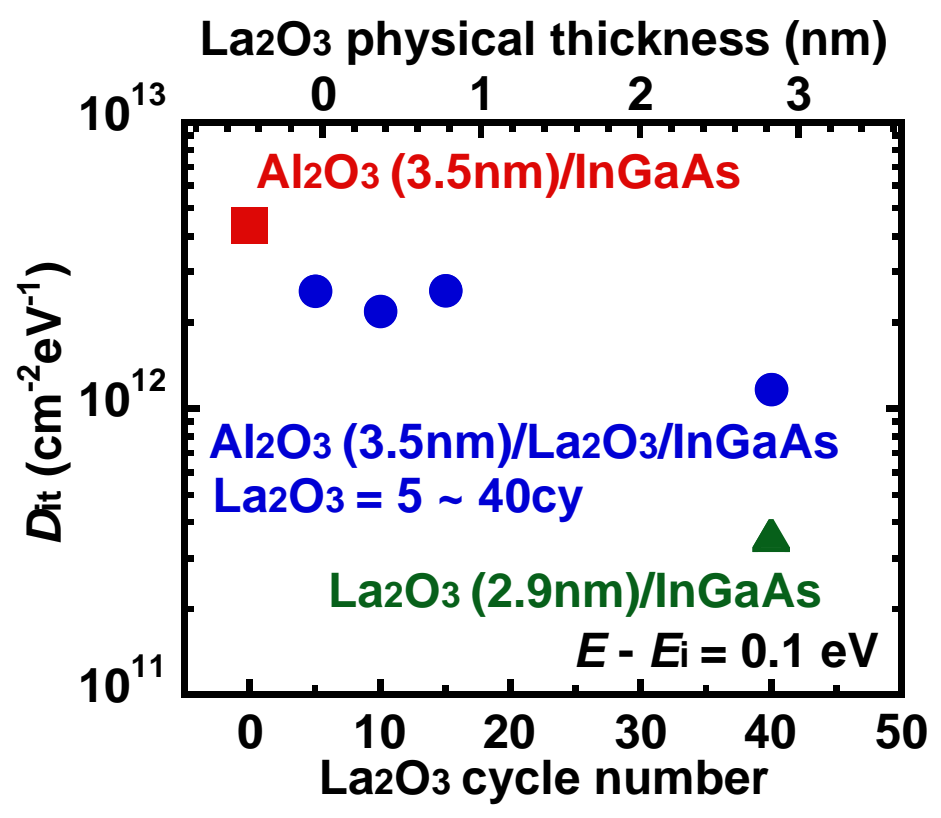

Fig. 8 (S. Takagi) 


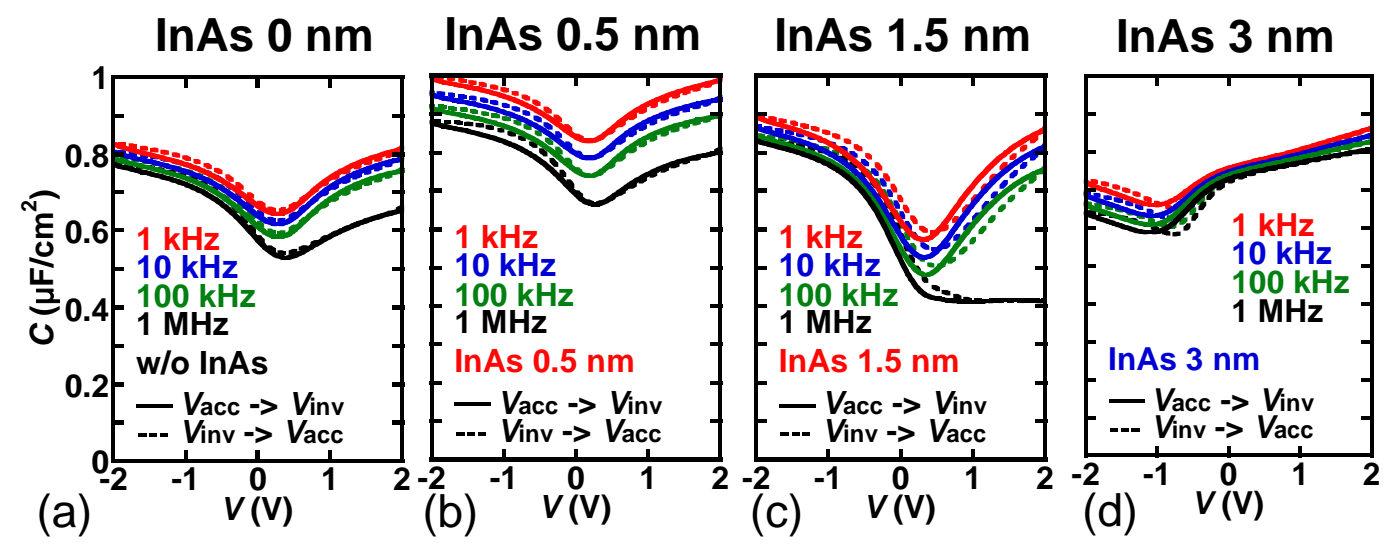

Fig. 9 (S. Takagi)

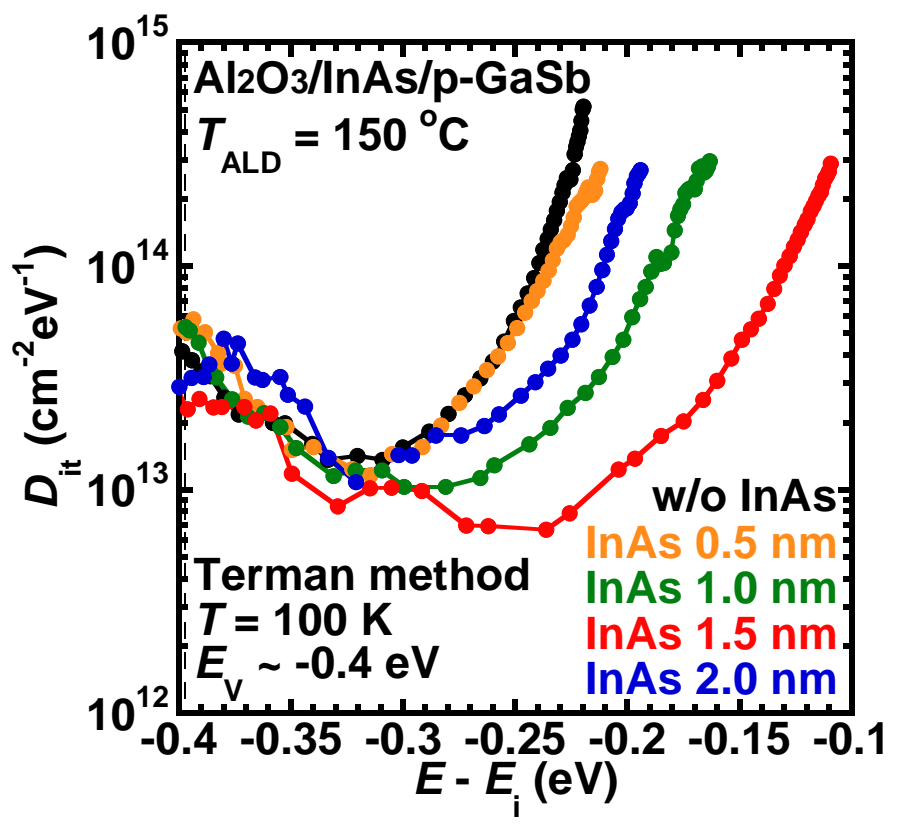

Fig. 10 (S. Takagi) 


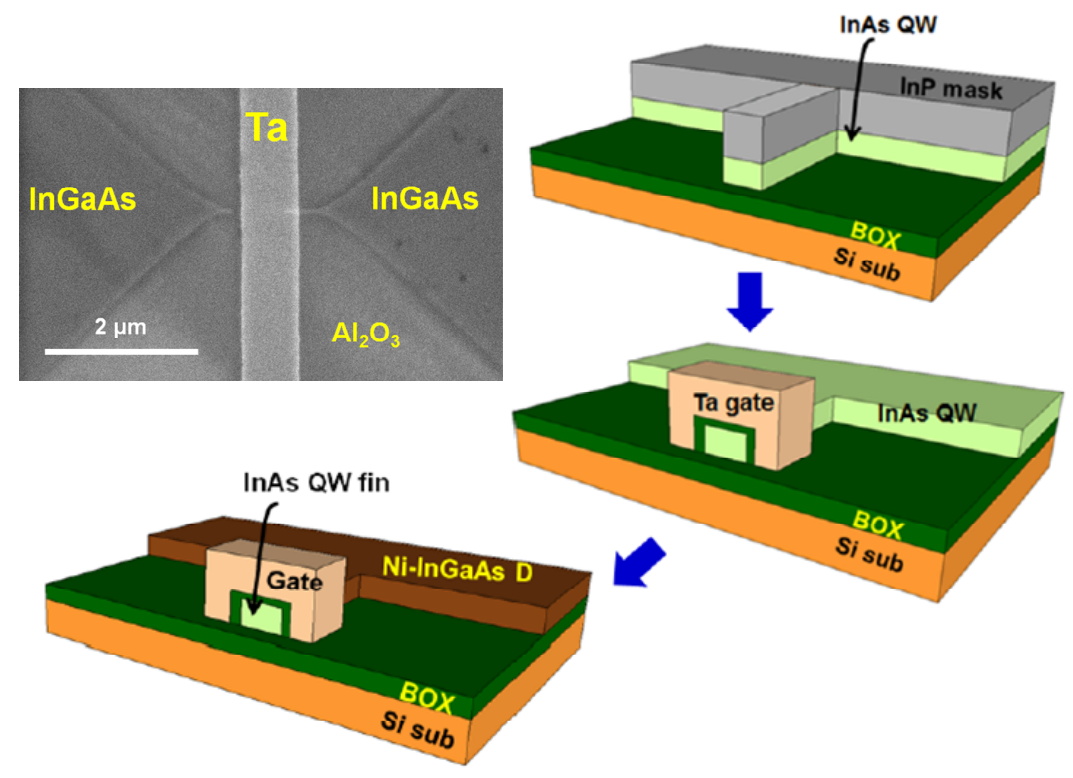

Fig. 11 (S. Takagi)
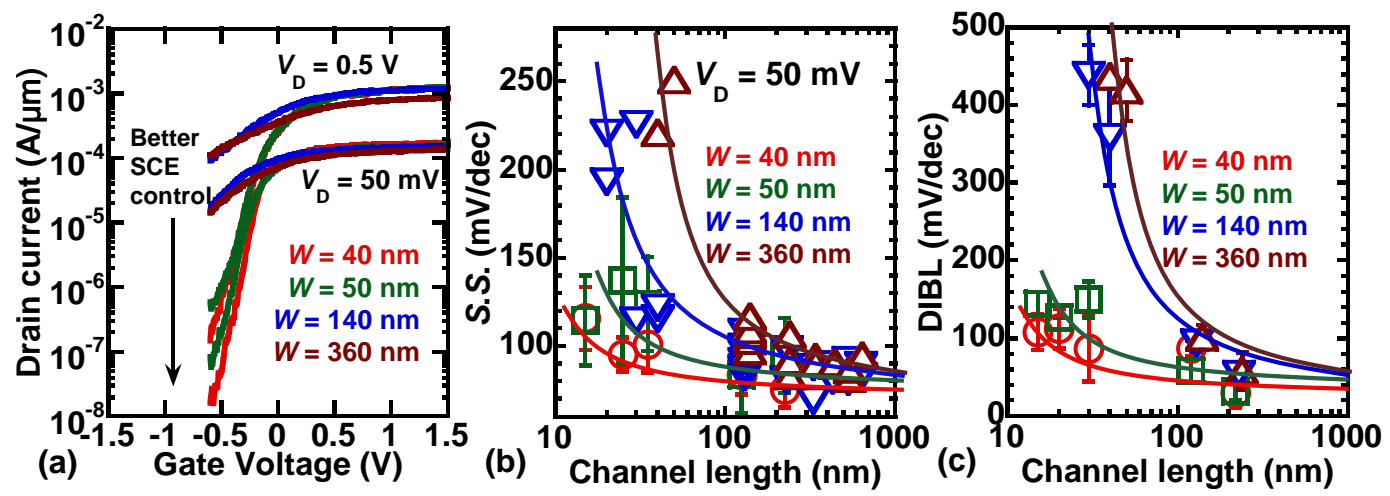

Fig. 12 (S. Takagi) 
Intel, IEDM2011 (Tri-gate)

Purdue, IEDM2012 (GAA)

Teledyne, IEDM2012 (Planar)

Sematech, IEDM2012 (Planar)

UCSB, VLSI2013 (Planar)

MIT, IEDM2013 (Planar)

Sematech, IEDM2013 (Fin)

TSMC Europe, IEDM2013 (Planar)
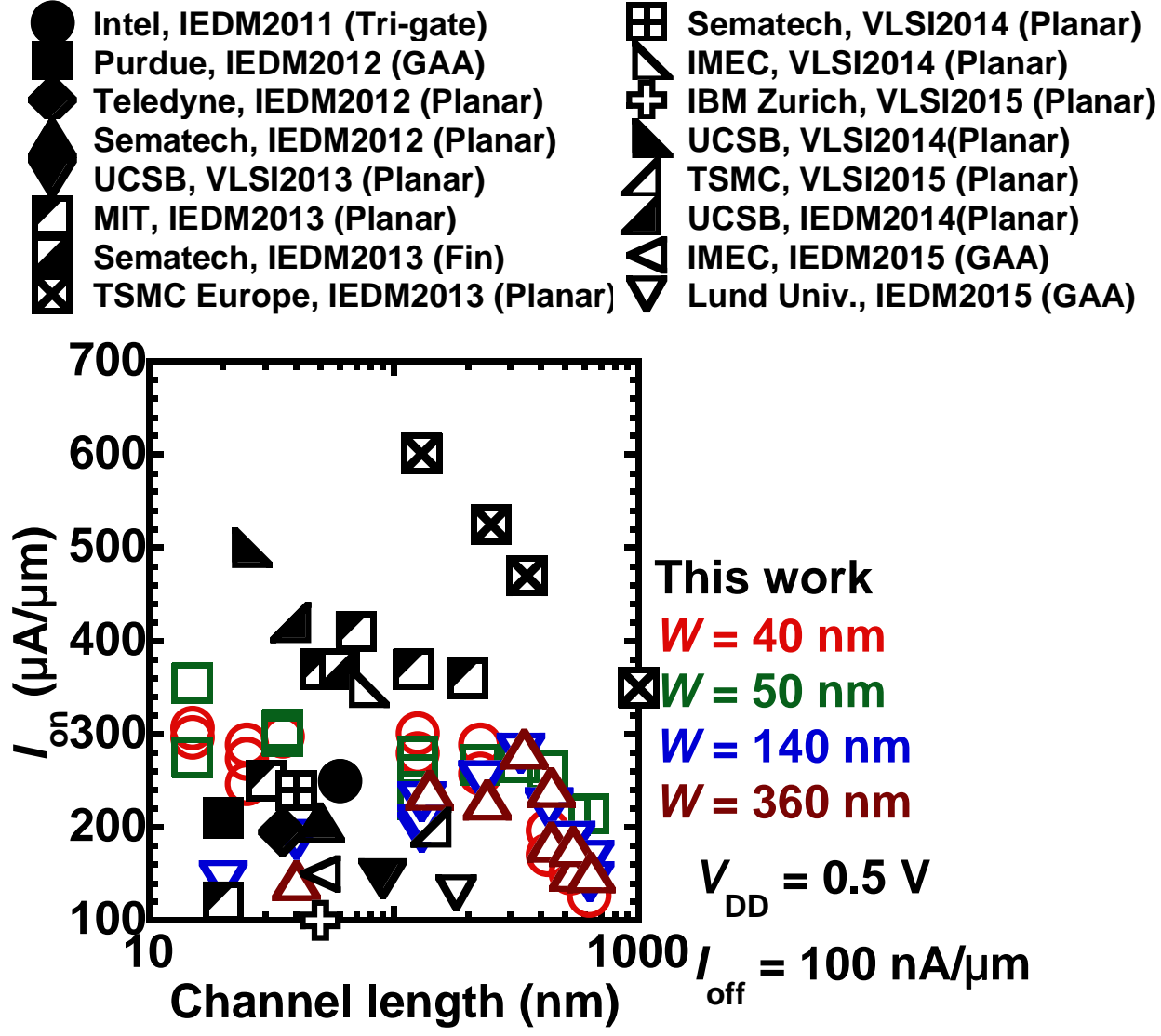

Fig. 13 (S. Takagi)

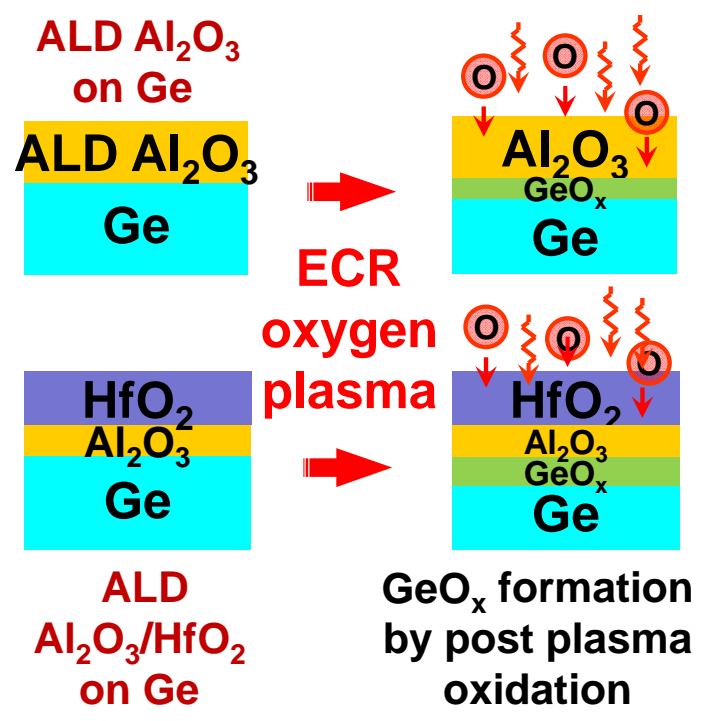

Fig. 14 (S. Takagi) 


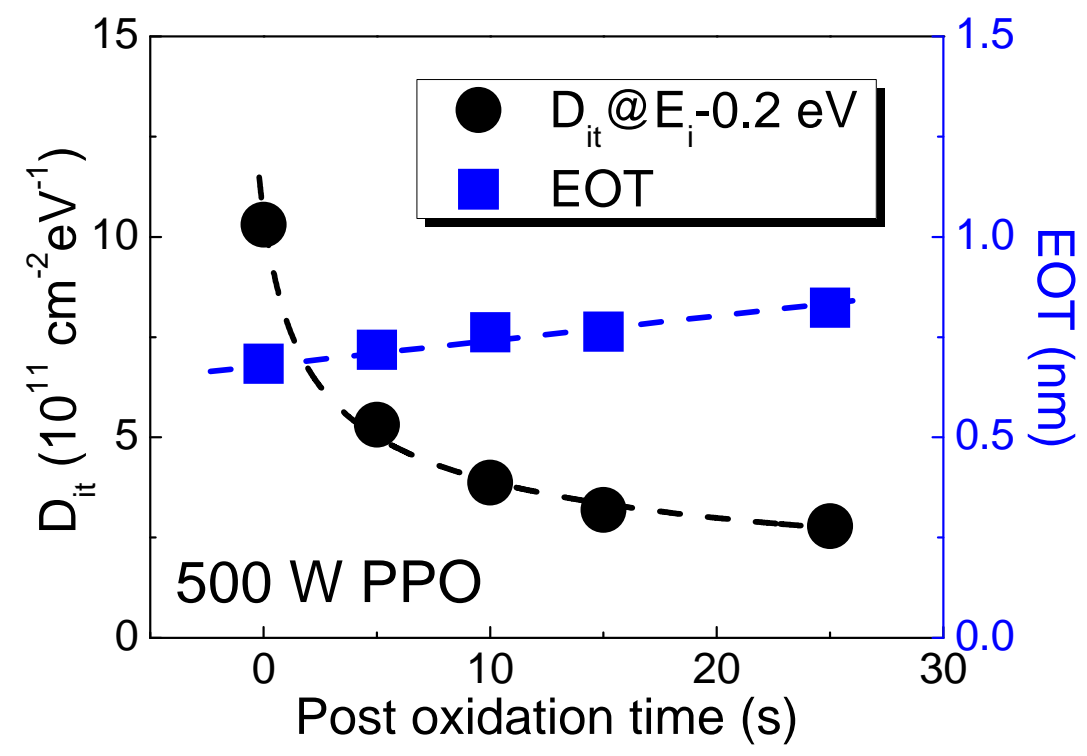

Fig. 15 (S. Takagi)
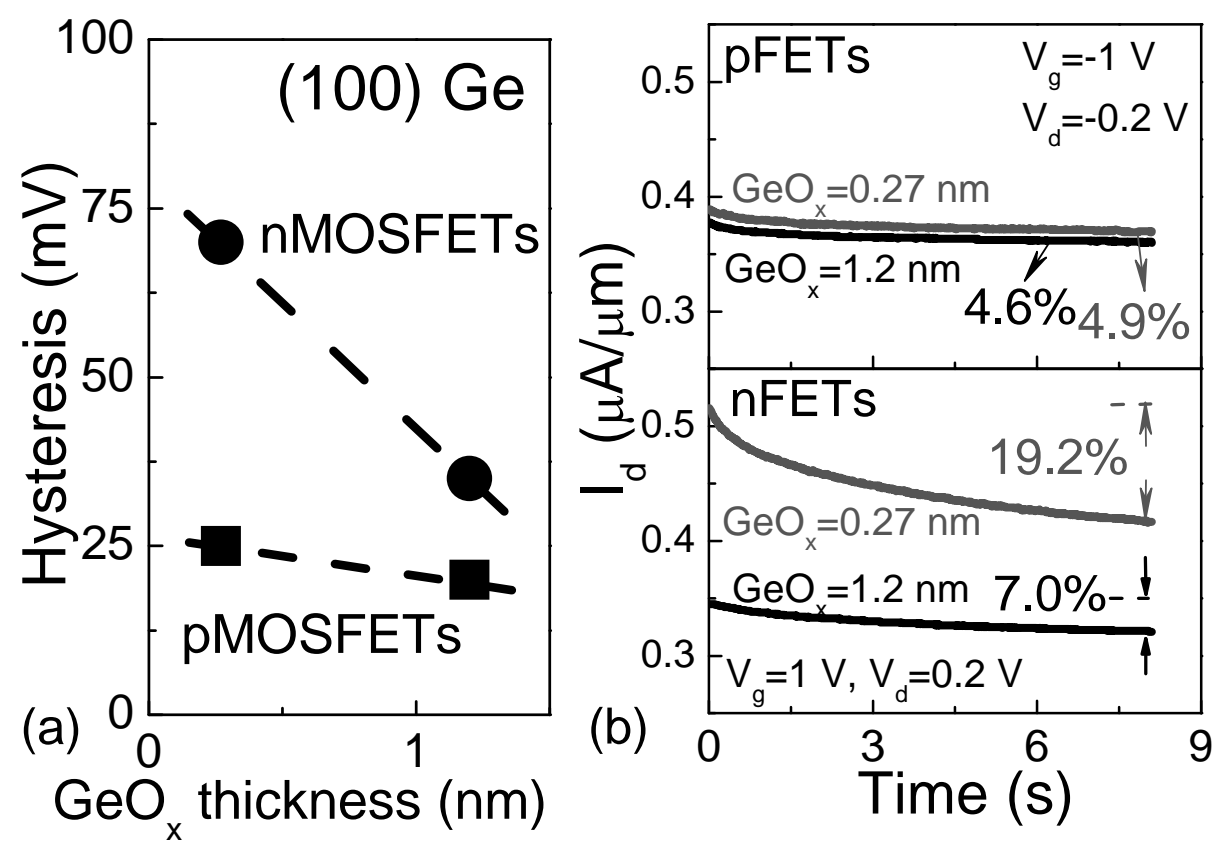

Fig. 16 (S. Takagi) 


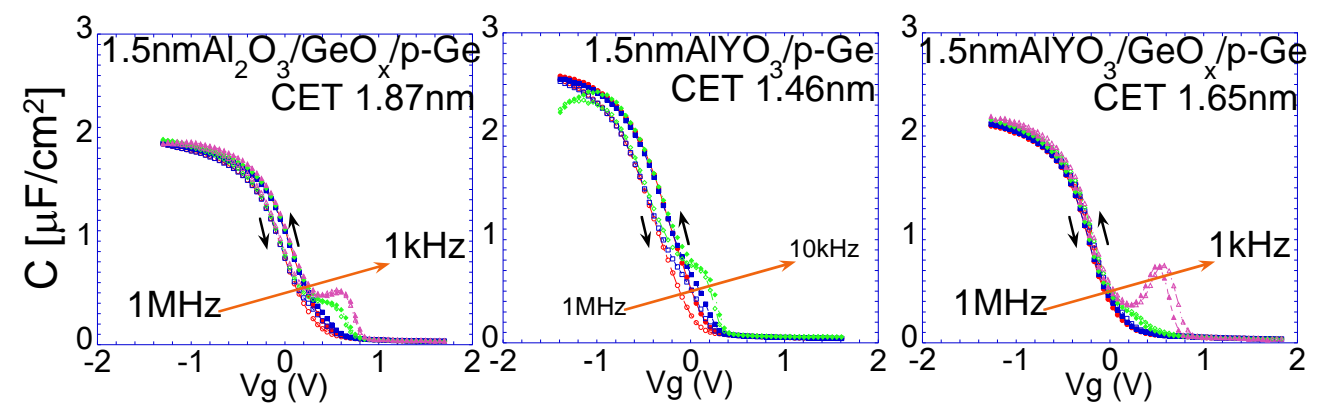

Fig. 17 (S. Takagi)
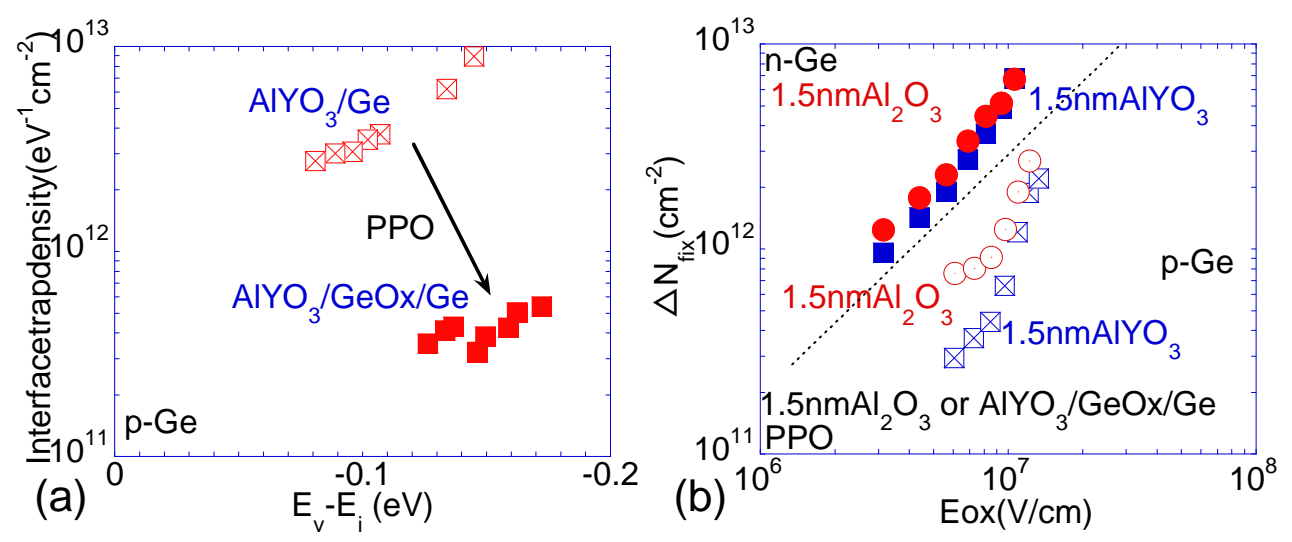

Fig. 18 (S. Takagi) 


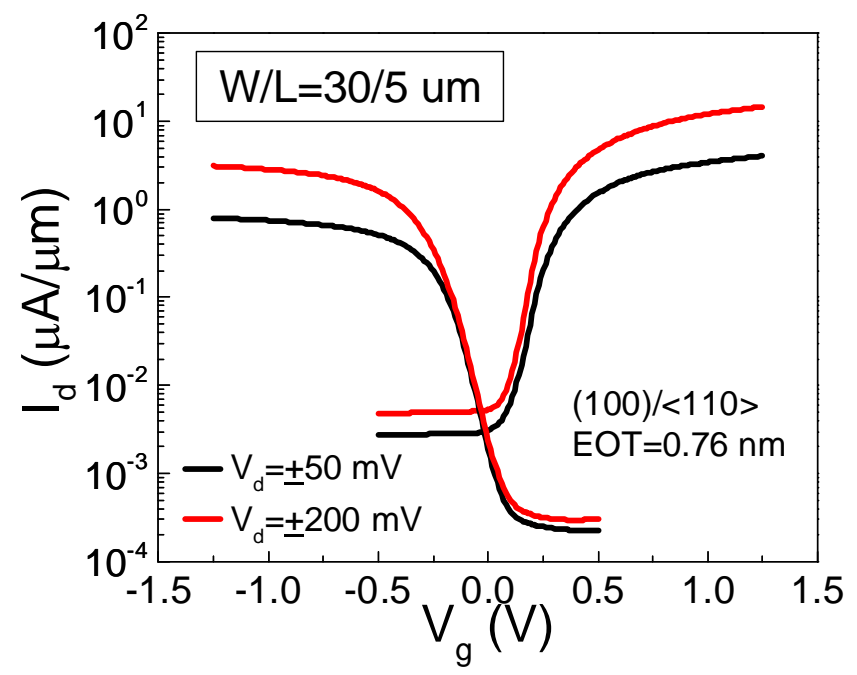

Fig. 19 (S. Takagi)
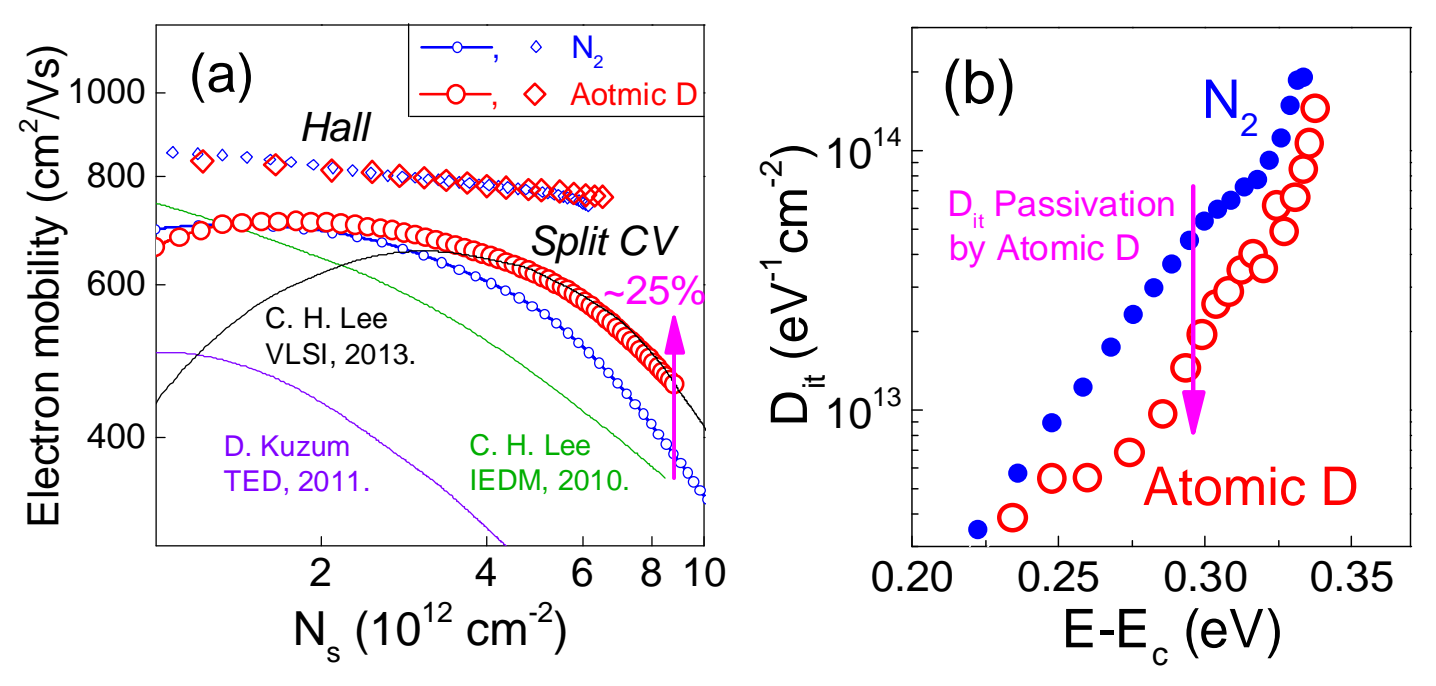

Fig. 20 (S. Takagi) 


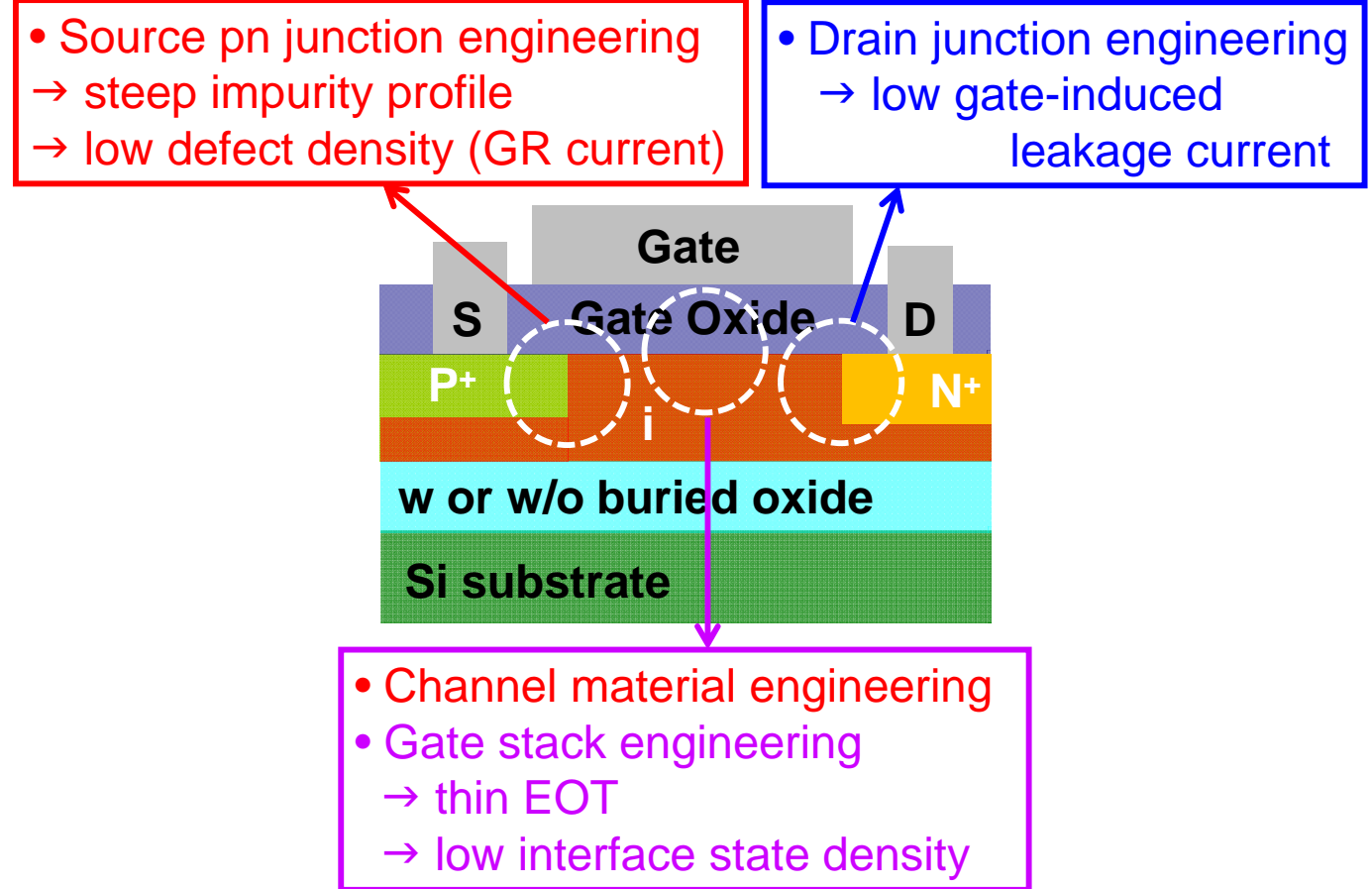

Fig. 21 (S. Takagi)

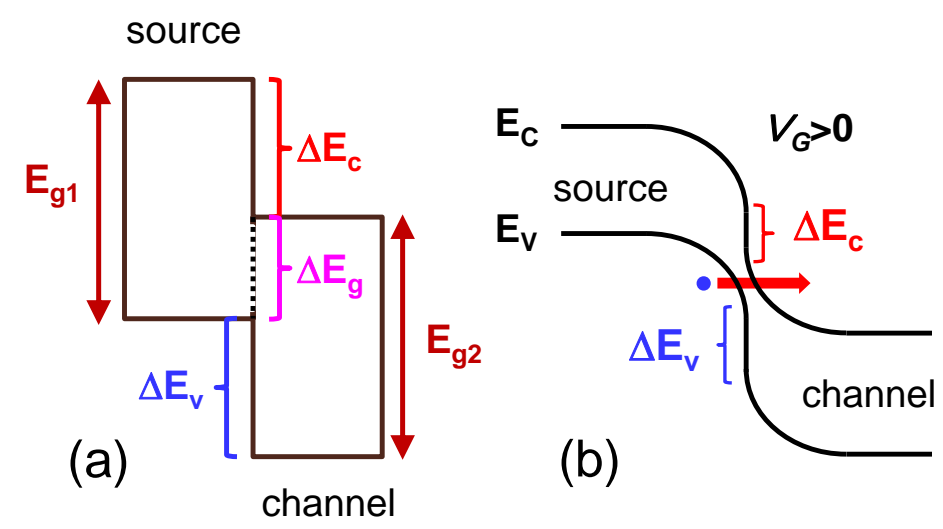

Fig. 22 (S. Takagi) 


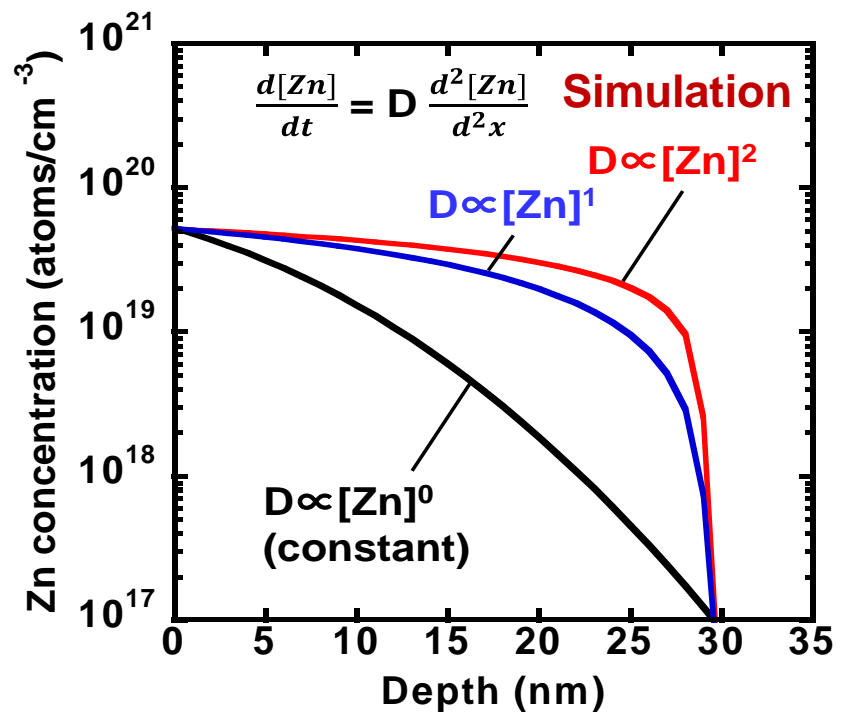

Fig. 23 (S. Takagi)
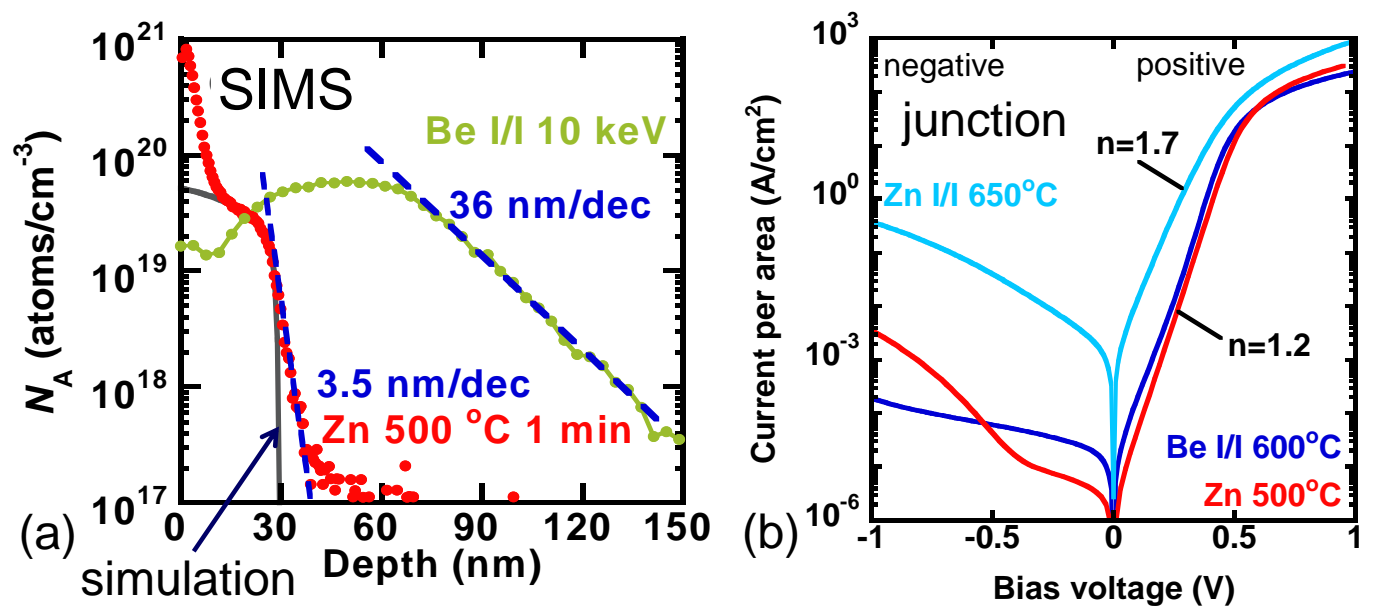

Fig. 24 (S. Takagi) 

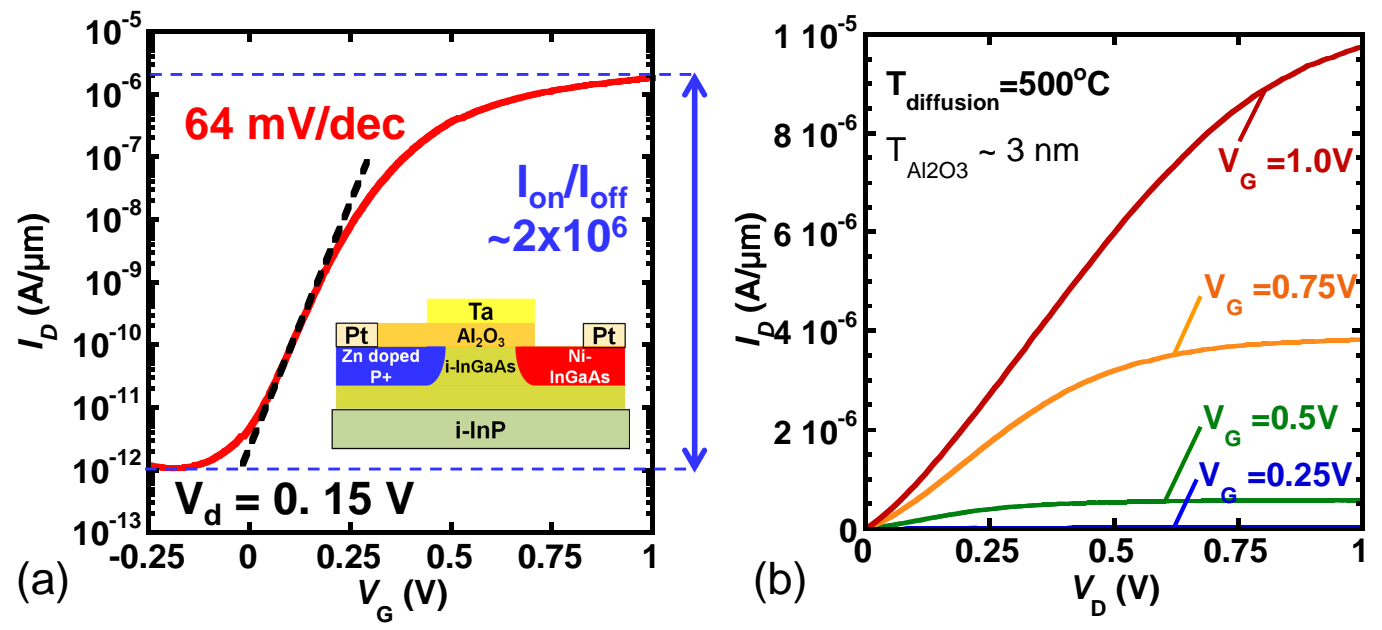

Fig. 25 (S. Takagi)

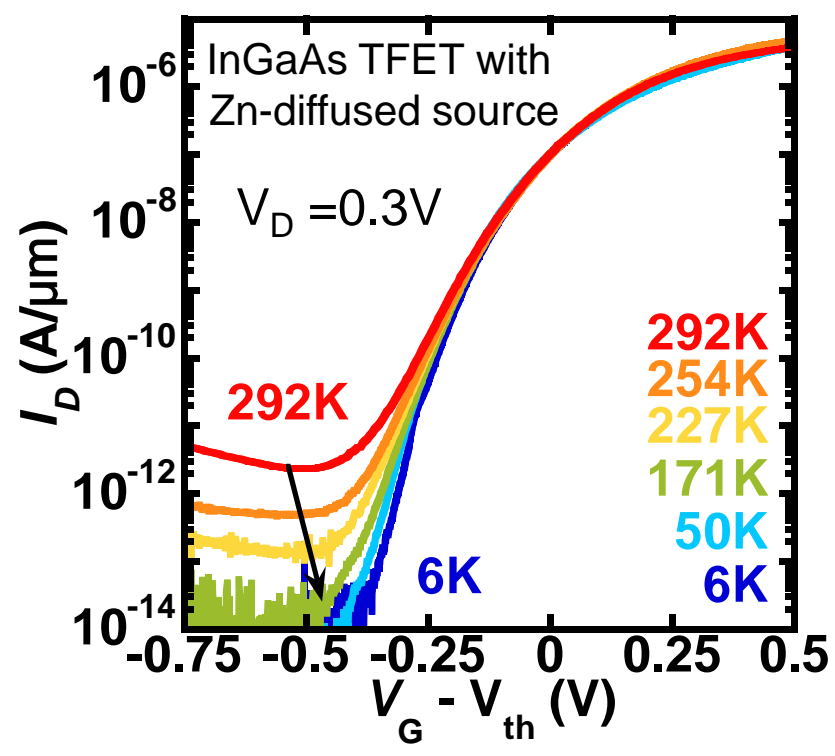

Fig. 26 (S. Takagi) 


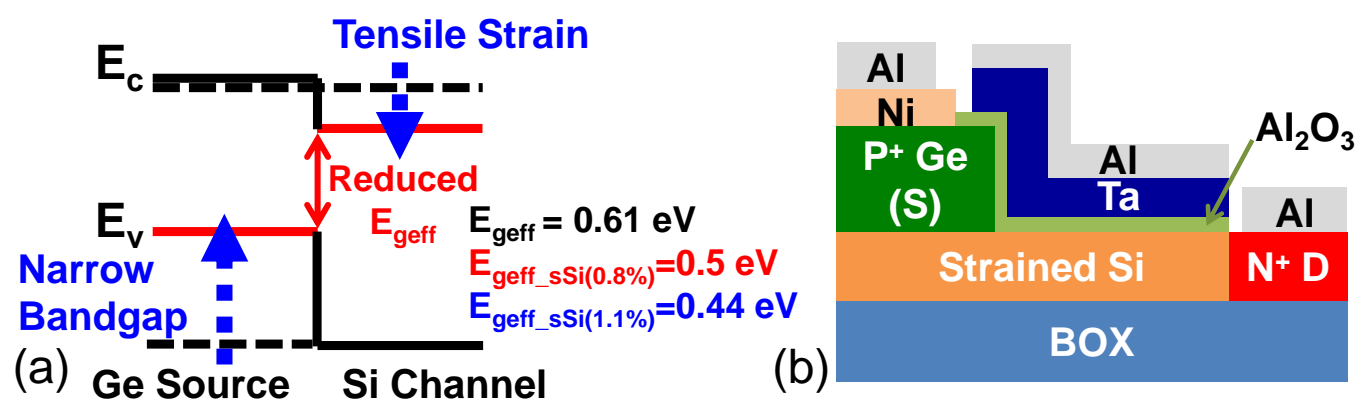

Fig. 27 (S. Takagi)

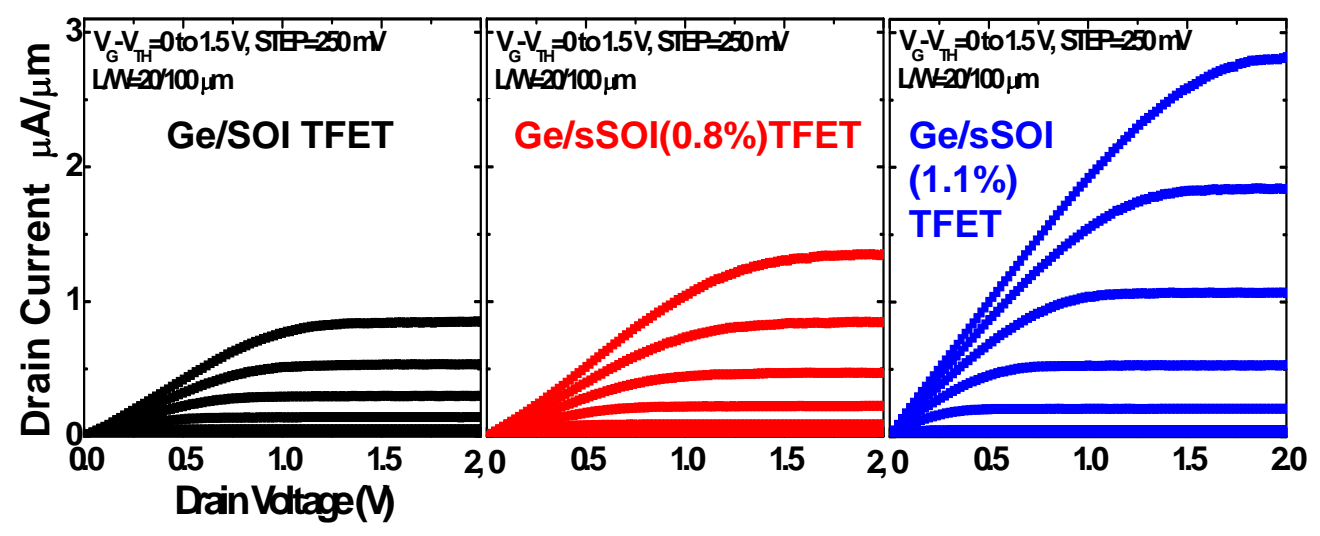

Fig. 28 (S. Takagi)

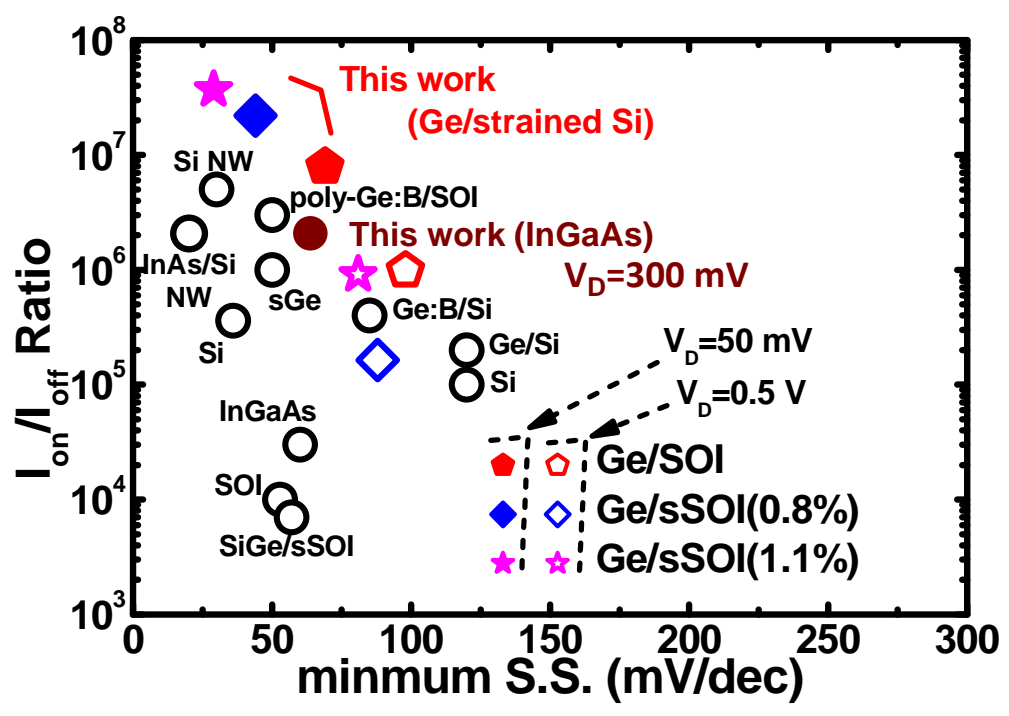

Fig. 29 (S. Takagi) 